\title{
MARKET POWER AND WELFARE IN ASYMMETRIC DIVISIBLE GOOD AUCTIONS
}

\author{
Carolina Manzano
}

Xavier Vives 
The Public-Private Sector Research Center is a Research Center based at IESE Business School. Its mission is to develop research that analyzes the relationships between the private and public sectors primarily in the following areas: regulation and competition, innovation, regional economy and industrial politics and health economics.

Research results are disseminated through publications, conferences and colloquia. These activities are aimed to foster cooperation between the private sector and public administrations, as well as the exchange of ideas and initiatives.

The sponsors of the Public-Private Sector Research Center are the following:

- Ajuntament de Barcelona

- Departament d' Economia i Coneixement de la Generalitat de Catalunya

- Departament d' Empresa i Ocupació de la Generalitat de Catalunya

- Diputació de Barcelona

- EVERIS

- Fundació AGBAR

- Institut Català de les Empreses Culturals (ICEC)

- PricewaterhouseCoopers

- Sanofi

The contents of this publication reflect the conclusions and findings of the individual authors and not the opinions of the Center's sponsors. 


\title{
Market Power and Welfare in Asymmetric Divisible Good Auctions*
}

\author{
Carolina Manzano \\ Universitat Rovira i Virgili and CREIP
}

July 16, 2018

\author{
Xavier Vives \\ IESE Business School
}

\begin{abstract}
We analyze a divisible good uniform-price auction that features two groups each with a finite number of identical bidders and present conditions under which a unique privately revealing equilibrium exists. We derive novel comparative static results highlighting that increases in transaction costs and noise in the signals of a group reinforce each other in making demand schedules of both groups steeper. If the correlation of values of the groups raises, as in a crisis situation, then the illiquidity effect is further reinforced. A "stronger" bidding group -which has more precise private information, faces lower transaction costs, and is more oligopsonistic- has more market power (price impact) and so will behave competitively only if it receives a higher per capita subsidy rate. When the strong group values the asset no less than the weak group, the expected deadweight loss increases with the quantity auctioned and also with the degree of payoff asymmetries. Price impact and the deadweight loss may be negatively associated and market integration may reduce welfare. The results are consistent with the available empirical evidence.

KEYWORDS: demand/supply schedule competition, private information, liquidity auctions, Treasury auctions, electricity auctions, market integration.
\end{abstract}

JEL: D44, D82, G14, E58

*For helpful comments we are grateful to Roberto Burguet, Maryam Farboodi, Vitali Gretschko, Jakub Kastl, Leslie Marx, Meg Meyer, Antonio Miralles, Stephen Morris, Andrea Prat, and Tomasz Sadzik as well as seminar participants at the BGSE Summer Forum, Chicago, Columbia, Como Information Economics Workshop, EARIE, ESSET, Federal Reserve Board, Jornadas de Economía Industrial, Northwestern, Princeton, UPF, Queen Mary Theory Workshop, Stanford, UC Berkeley, UC San Diego, and Wharton. We are also indebted to Jorge Paz for excellent research assistance and to the Spanish Ministry of Economy and Competitiveness (ECO2016-75410-P for Manzano and ECO2015-63711-P for Vives) and to the Generalitat de Catalunya, AGAUR grant 2014 SGR 1496 for Vives for financial support. 


\section{Introduction}

Divisible good auctions are common in many markets, including government bonds, liquidity (refinancing operations), electricity, and emission markets. ${ }^{1}$ In those auctions, both market power (price impact) and asymmetries among the participants are important; asymmetries can make price impact relevant even in large markets. However, theoretical work in this area has been hampered by the difficulties of dealing with bidders that are asymmetric, have market power, and are competing in terms of demand or supply schedules in the presence of private information. ${ }^{2}$ This paper helps to fill that research gap by analyzing asymmetric uniformprice auctions in which there are two groups of bidders. Our aims are to characterize the equilibrium, to perform comparative statics and welfare analysis (from the standpoint of revenue and deadweight loss), and finally to derive implications for policy.

Divisible good auctions are typically populated by heterogeneous participants in a concentrated market, and often we can distinguish a core group of bidders together with a fringe. The former are strong in the sense that they have better information, endure lower transaction costs, and are more oligopolistic (or oligopsonistic) than members of the fringe. As examples we discuss Treasury and liquidity auctions in addition to wholesale electricity markets. Uniform-price auctions are often used in Treasury, liquidity and electricity auctions.

Treasury auctions have bidders with significant market shares. That will be the case in most systems featuring a primary dealership, where participation is limited to a fixed number of bidders (this occurs, for example, in 29 out of 39 countries surveyed by Arnone and Iden 2003). A prime example are US Treasury auctions, which are uniform-price auctions since 1998. In these auctions, the top five bidders typically purchase close to half of US Treasury issues. ${ }^{3}$ Primary dealers underwent a substantial reduction going from 46 in 1998 to 23 presently. Those account for a very substantial portion of volume (from $69 \%$ to $88 \%$ of tendered quantities in the sample of Hortaçsu et al. (2016) for the years 2009-2013). Indirect bidders place their bids through the primary dealers and other direct bidders tender from 6 to $13 \%$.

Primary dealers enjoy an information advantage because they aggregate the information of indirect bidders and face lower transaction costs. ${ }^{4}$ Primary dealers bid systematically lower

\footnotetext{
${ }^{1}$ See Lopomo et al. (2011) for examples of such auctions.

${ }^{2} \mathrm{~A}$ difficulty found in indivisible good auctions is that closed form solutions for the equilibrium in asymmetric auctions are typically not available (even with independent private values). Consequently, the analysis of this type of auctions has posed many challenges and progress in this area has been sporadic (see, e.g., Hafalir and Krishna 2008 and Hubbard and Kirkegaard 2015).

${ }^{3}$ According to Euromoney, the top five commanded $58 \%$ of the market in 2016, up from $43 \%$ in 2006 ; and the top ten dealers 85\%. See also Malvey and Archibald (1998).

${ }^{4} \mathrm{~A}$ proxy for the information advantage of a primary dealer is the number of indirect bidders that go through
} 
prices than the other participants in the auction, according to Hortaçsu et al. (2016), not because they have a lower valuation of the securities but because they exercise market power. ${ }^{5}$

Armantier and Sbaï (2006) test for whether the bidders in French Treasury auctions are symmetric; these authors conclude that such auction participants can be divided into two distinct groups as a function of (a) their level of risk aversion and (b) the quality of their information about the value of the security to be sold. One small group consists of large financial institutions, which possess better information and are willing to take more risks. Kastl (2011) also finds evidence of two distinct groups of bidders in (uniform-price) Czech Treasury auctions. Other papers that report asymmetries between bidders in Treasury auctions include, among others, Umlauf (1993) for Mexico, Bjonnes (2001) for Norway, and Hortaçsu and McAdams (2010) for Turkey. All this evidence points to the prevalence of joint trader heterogeneity in terms of transaction costs and quality of information. Hamao and Jegadeesh (1998) show that the major participants in the Japanese Government bond primary market can be divided into two groups, Japanese and U.S. investment banks receiving (across groups) correlated signals. Their results indicate the existence of bid synchronization of the Japanese banks. These authors argue that a plausible explanation for this bidding behavior is the fact that the Japanese investment banks observe similar information or apply similar models to analyze information. Another possibility is that customer demand for bonds is correlated among investment banks. This would explain the synchronization then since the dealers place the bids on behalf of the customers, with the Japanese banks jointly bidding aggressively in some auctions and not so in others. Cao and Lu (2004) also find bid synchronization among large bidders, but not among small bidders, in Canadian Treasury auctions.

Bindseil et al. (2009) and Cassola et al. (2013) find that the heterogeneity of bidders in liquidity auctions is relevant. Cassola et al. (2013) analyze the evolution of bidding data from the European Central Bank's weekly refinancing operations before and during the early part of the financial crisis. The authors show that effects of the 2007 subprime market crisis were heterogeneous among European banks, and they conclude that the significant shift in bidding behavior after 9 August 2007 may reflect a change in the cost of short-term funding on the interbank market and/or a strategic response to other bidders. In particular, Cassola et al. (2013) find that one third of bidders experienced no change in their costs of short-term funds from alternative sources; this means that their altered bidding behavior was mainly strategic:

this dealer. For evidence from Canadian Treasury auctions, see Hortaçsu and Kastl (2012); for a theoretical model see Boyarchenko et al. (2015).

${ }^{5}$ Experimental work has found also substantial demand reduction in uniform-price auctions (see e.g. Kagel and Levin 2001; Engelbrecht-Wiggans et al. 2006). 
bids were increased as a best response to the higher bids of rivals. ${ }^{6}$

Our paper makes progress within the linear-Gaussian family of models by incorporating bidders' asymmetries with regard to payoffs and information. We model a uniform-price auction where asymmetric strategic bidders compete in terms of demand schedules for an inelastic supply (we can easily accommodate supply schedule competition for an inelastic demand). We consider a model in which the equilibrium is privately revealing, that is, where the signal received by a trader and the price are a sufficient statistic for the trader. This allows us to concentrate the analysis in the inefficiencies derived from private information and market power with no information externality present. Bidders may differ in their valuations, transaction costs, and/or the precision of their private information. With an empirical basis, we reduce heterogeneity to two groups; within each group, agents are identical and receive the same signal. ${ }^{7}$ This information structure is consistent with the above mentioned empirical evidence in Hamao and Jegadeesh (1998) and Cao and Lu (2004) which tends to suggest the presence of a group with very correlated signals and high precision and another with low correlation and poor or uninformative signals. We seek to identify the conditions under which there exists a linear equilibrium with symmetric treatment of agents in the same group (i.e., we are looking for equilibria such that demand functions are both linear and identical among individuals of the same type). The equilibrium will be privately revealing and after showing that any such equilibrium must be unique, we derive novel comparative statics results.

More specifically, our analysis establishes that the number of group members, the transactions costs, the extent to which bidders' valuations are correlated, and the precision of private information affect the sensitivity of traders' demands to private information and prices. When valuations are more correlated, traders react less to the private signal and to the price. We also find that the relative price impact of a group increases with the precision of its private information and decreases with its transaction costs. Furthermore, if the transaction costs of a group increase, then the traders of the other group respond strategically by diminishing their reaction to private information and submitting steeper schedules. This result is consistent with the empirical findings of Cassola et al. (2013) in European post-crisis liquidity auctions. It is key that increases in transaction costs, correlation of values, and noise in the signals, all descrip-

\footnotetext{
${ }^{6}$ Bidder asymmetry has also been found in procurement markets, including school milk (Porter and Zona 1999; Pesendorfer 2000) and public works (Bajari 1998).

${ }^{7}$ In case one group does not receive a signal we reproduce the information structure in Grossman and Stiglitz (1980) of uninformed and informed traders. Goldstein and Yang (2015) propose an extension of this model within a competitive framework with two groups of informed traders, each of which observing a piece of uncertainty of the value of the traded asset. The authors show that acquiring information about different components of fundamentals can be complementary.
} 
tive of a crisis situation, impinge in the direction of steepening demand schedules and illiquidity. We find also that if a group of traders is stronger in the sense that its private information is more precise, its transaction costs are lower, and it is more oligopolistic, then the members of that group react more (than do the bidders of the other group) to the private signal and to the price, and have more price impact. This result is consistent with the evidence in Hortaçsu et al. (2016) that primary dealers exercise market power.

We also find that, when the expected valuations between groups differ, the auction's expected revenue needs not be decreasing in the transaction costs of bidders, the noise in their signals, or the correlation of values. These findings contrast with the results obtained when groups are symmetric. We bound the expected revenue of the auction between the revenues of auctions involving extremal yet symmetric groups.

In this paper we consider large markets and find that, if there is both a small and a large group of bidders, then the former (oligopsonistic) group has more price impact and yet even the latter (large) group does not behave competitively since it retains some price impact due to incomplete information. We also prove that the equilibrium under imperfect competition converges to a price-taking equilibrium in the limit as the number of traders (of both groups) becomes large. Interestingly, the expected competitive price may be lower than the expected oligopoly price when the quantity auctioned is low and the group that values the asset more is weak.

Finally, we provide a welfare analysis. Toward that end, we characterize the deadweight loss at the equilibrium and show how a subsidy scheme may induce an efficient allocation. We find that if one group is stronger (as previously defined), then it should garner a higher per capita subsidy rate; the reason is that traders in the stronger group will behave more strategically and so must be compensated more to become competitive. The paper also underscores how the bidder heterogeneity (in terms of information, preferences, or group size documented in previous work) may increase deadweight losses. In particular, when the strong group values the asset at least as much as the weak group, the deadweight loss increases with the quantity auctioned and also with the degree of payoff asymmetries. We find also that price impact need not be positively correlated with deadweight losses under asymmetry as implicitly assumed usually in applied work. Furthermore, we provide conditions under which market integration increases or decreases welfare. It is shown, for example, that market integration is always welfare improving if bidders behave competitively or if the bidder groups are symmetric. However, the result does not hold if bidders have market power, the amount auctioned is large, and the groups asymmetric. In this case gains from trade of integration are overwhelmed by the inefficiency generated by group asymmetries and price impact.

Our work is related to the literature on divisible good auctions. Results in symmetric pure 
common value models have been obtained by Wilson (1979), Back and Zender (1993), and Wang and Zender (2002), among others. ${ }^{8}$ Kastl (2011) extends the Wilson model to consider discrete bids in an independent values context. This model is extended in Hortaçsu and Kastl (2012) and Hortaçsu et al. (2016).

Results in interdependent values models with symmetric bidders are obtained by Vives (2011, 2014) and Ausubel et al. (2014), for example. ${ }^{9}$ Vives (2011), while focusing on the tractable family of linear-Gaussian models, shows how increased correlation in traders' valuations increases the price impact of those traders. Bergemann et al. (2015) generalize the information structure in Vives (2011) while retaining the assumption of symmetry. Rostek and Weretka (2012) partially relax that assumption and replace it with a weaker "equicommonality" assumption on the matrix correlation among the agents' values. ${ }^{10}$ Babus and Kondor (2017) examine the effect of trade decentralization comparing a centralized market as described in Vives (2011) and a decentralized market in which dealers can engage in bilateral transactions with other dealers. The paper shows that the effect of trade decentralization on welfare and liquidity is in general ambiguous. Du and Zhu (2015) consider a dynamic auction model with ex post equilibria. For the case of complete information, progress has been made in divisible good auction models by characterizing linear supply function equilibria (e.g., Klemperer and Meyer 1989; Akgün 2004; Anderson and $\mathrm{Hu} 2008$ ). An exception that incorporates incomplete information is the paper by Kyle (1989), who considers a Gaussian model of a divisible good double auction in which some bidders are privately informed and others are uninformed. ${ }^{11}$ Sadzik and Andreyanov (2016) study the design of robust exchange mechanisms in a two-type model similar to the one we

\footnotetext{
${ }^{8}$ Wilson (1979) compares a uniform-price auction for a divisible good with an auction in which the good is treated as an indivisible good; he finds that the price can be significantly lower if bidders are allowed to submit bid schedules rather than a single bid price. That work is extended by Back and Zender (1993), who compare a uniform-price auction with a discriminatory auction. These authors demonstrate the existence of equilibria in which the seller's revenue in a uniform-price auction can be much lower than the revenue obtained in a discriminatory auction. According to Wang and Zender (2002), if supply is uncertain and bidders are risk averse, then there may exist equilibria of a uniform-price auction that yield higher expected revenue than that from a discriminatory auction.

${ }^{9}$ Ausubel et al. (2014) find that, in symmetric auctions with decreasing linear marginal utility, the seller's revenue is greater in a discriminatory auction than in a uniform-price auction. Pycia and Woodward (2016) demonstrate that a discriminatory pay-as-bid auction is revenue-equivalent to the uniform-price auction provided that supply and reserve prices are set optimally.

${ }^{10}$ This assumption states that the sum of correlations in each column of this matrix (or, equivalently, in each row) is the same and that the variances of all traders' values are also the same. Unlike our model, Rostek and Weretka's (2012) model maintains the symmetry assumption as regards transaction costs and the precision of private signals. The equilibrium they derive is therefore still symmetric because all traders use identical strategies.

${ }^{11}$ Lambert et al. (2016) extend the Kyle (1985) model to general correlation patterns among random variables.
} 
present here. We present a series of novel comparative statics results characterizing how the asymmetries of market participants affect their bidding behavior.

Despite the importance of bidder asymmetry, results in multi-unit auctions have been difficult to obtain. As a consequence, most papers that deal with this issue focus on auctions for a single item. In sealed-bid, first-price, single-unit auctions, an equilibrium exists under quite general conditions (Lebrun 1996; Maskin and Riley 2000a; Athey 2001; Reny and Zamir 2004). Uniqueness is explored in Lebrun (1999) and Maskin and Riley (2003). Maskin and Riley (2000b) study asymmetric auctions, and Cantillon (2008) shows that the seller's expected revenue declines as bidders become less symmetric. On the multi-unit auction front, progress in establishing the existence of monotone equilibria has been made by McAdams (2003, 2006); those papers address uniform-price auctions characterized by multi-unit demand, interdependent values and independent types. ${ }^{12}$ Reny (2011) stipulates more general existence conditions that allow for infinite-dimensional type and action spaces; these conditions apply to uniformprice, multi-unit auctions with weakly risk-averse bidders and interdependent values (and where bids are restricted to a finite grid).

The rest of our paper is organized as follows. Section 2 outlines the model. Section 3 characterizes the equilibrium, analyzes its existence and uniqueness, and derives comparative statics results. We develop the welfare analysis in Section 4 and address large markets in Section 5. Section 6 deals with supply function competition and Section 7 concludes. Proofs are gathered in Appendix A and supplementary material in Appendix B.

\section{The model}

Traders, of whom there are a finite number, face an inelastic supply for a risky asset. Let $Q$ denote the aggregate quantity supplied in the market. In this market there are buyers of two types: type 1 and type 2 . Suppose that there are $n_{i}$ traders of type $i, i=1,2$. In that case, if the asset's price is $p$, then the profits of a representative type- $i$ trader who buys $x_{i}$ units of the asset are given by

$$
\pi_{i}=\left(\theta_{i}-p\right) x_{i}-\lambda_{i} x_{i}^{2} / 2
$$

So, for any trader of type $i$, the marginal benefit of buying $x_{i}$ units of the asset is $\theta_{i}-\lambda_{i} x_{i}$, where $\theta_{i}$ denotes the valuation of the asset and $\lambda_{i}>0$ reflects an adjustment for transaction costs or opportunity costs (or a proxy for risk aversion). Traders maximize expected profits and

\footnotetext{
${ }^{12}$ McAdams (2006) uses a discrete bid space and atomless types to show that, with risk neutral bidders, monotone equilibria exist. The demonstration is based on checking that the single-crossing condition used by Athey (2001) for the single-object case extends to multi-unit auctions.
} 
submit demand schedules, after which the auctioneer selects a price that clears the market. The case of supply schedule competition for inelastic demand is easily accommodated by considering negative demands $\left(x_{i}<0\right)$ and a negative inelastic supply $(Q<0)$. In this case, a producer of type $i$ has a quadratic production cost $-\theta_{i} x_{i}+\lambda_{i} x_{i}^{2} / 2$.

We assume that $\theta_{i}$ is normally distributed with mean $\bar{\theta}_{i}$ and variance $\sigma_{\theta}^{2}, i=1,2$. The random variables $\theta_{1}$ and $\theta_{2}$ may be correlated, with correlation coefficient $\rho \in[0,1]$. Therefore, $\operatorname{cov}\left(\theta_{1}, \theta_{2}\right)=\rho \sigma_{\theta}^{2} \cdot{ }^{13}$ All type- $i$ traders receive the same noisy signal $s_{i}=\theta_{i}+\varepsilon_{i}$, where $\varepsilon_{i}$ is normally distributed with null mean and variance $\sigma_{\varepsilon_{i}}^{2}$. Error terms in the signals are uncorrelated across groups $\left(\operatorname{cov}\left(\varepsilon_{1}, \varepsilon_{2}\right)=0\right)$ and are also uncorrelated with valuations of the asset $\left(\operatorname{cov}\left(\varepsilon_{i}, \theta_{j}\right)=0, i, j=1,2\right)$.

In our model, two traders of distinct types may differ in several respects:

- different willingness to possess the asset $\left(\theta_{1} \neq \theta_{2}\right)$,

- different transaction costs $\left(\lambda_{1} \neq \lambda_{2}\right)$, and/or

- different levels of precision of private information $\left(\sigma_{\varepsilon_{1}}^{2} \neq \sigma_{\varepsilon_{2}}^{2}\right)$.

Applications of this model are Treasury auctions and liquidity auctions. For Treasury auctions, $\theta_{i}$ is the private value of the securities to a bidder of type $i$; that value incorporates not only the resale value but also idiosyncratic elements as different liquidity or portfolio immunization needs of bidders in the two groups. Financial intermediaries may assign different values to the Treasury instruments according to their use as collateral. In particular, primary dealers may attach a value to a bond beyond resale value to be used as collateral in operations with the Fed. For liquidity auctions, $\theta_{i}$ is the price (or interest rate) that group $i$ commands in the secondary interbank market (which is over-the-counter). Here $\lambda_{i}$ reflects the structure of a counterparty's pool of collateral in a repo auction. A bidder bank prefers to offer illiquid collateral to the central bank in exchange for funds; as allotments increase, however, the bidder must offer more liquid types of collateral which have a higher opportunity cost. This yields a declining marginal valuation. ${ }^{14}$

\section{Equilibrium}

Denote by $X_{i}$ the strategy of a type- $i$ bidder, $i=1,2$, which is a mapping from the signal space to the space of demand functions. Thus, $X_{i}\left(s_{i}, \cdot\right)$ is the demand function of a type- $i$ bidder

\footnotetext{
${ }^{13}$ The value of $\rho$ will depend of the type of security. In this sense, Bindseil et al. (2009) argue that the common value component is less important in a central bank repo auction than in a T-bill auction.

${ }^{14}$ See Ewerhart et al. (2010).
} 
that corresponds to a given signal $s_{i}$. Given her signal $s_{i}$, each bidder in a Bayesian equilibrium chooses a demand function that maximizes her conditional profit (while taking as given the other traders' strategies). We assume that there is a unique market clearing price given the submitted demand schedules and signal realizations. ${ }^{15}$ Our attention will be restricted to anonymous linear Bayesian equilibria in which strategies are linear and identical among traders of the same type (for short, equilibria).

Definition. An equilibrium is a linear Bayesian equilibrium such that the demand functions for traders of type $i, i=1,2$, are identical and equal to

$$
X_{i}\left(s_{i}, p\right)=b_{i}+a_{i} s_{i}-c_{i} p
$$

where $b_{i}, a_{i}$, and $c_{i}$ are constants.

The equilibrium is characterized in Subsection 3.1, its comparative static properties in Subsection 3.2, and equilibrium quantities in Subsection 3.3.

\subsection{Equilibrium characterization}

Consider a trader of type $i$. If rival's strategies are linear and identical among traders of the same type and if the market clears, that is, if $\left(n_{i}-1\right) X_{i}\left(s_{i}, p\right)+x_{i}+n_{j} X_{j}\left(s_{j}, p\right)=Q$, for $j=1,2$ and $j \neq i$, then this trader faces the residual inverse supply $p=I_{i}+d_{i} x_{i}$, where $I_{i}=\left(\left(n_{i}-1\right)\left(b_{i}+a_{i} s_{i}\right)+n_{j}\left(b_{j}+a_{j} s_{j}\right)-Q\right) /\left(\left(n_{i}-1\right) c_{i}+n_{j} c_{j}\right)$ and $d_{i}=1 /\left(\left(n_{i}-1\right) c_{i}+n_{j} c_{j}\right)$. The slope of residual supply $\left(d_{i}\right)$ is an index of the trader's market power or price impact. ${ }^{16}$ Indeed, by putting one more unit in the market a trader of type $i$ will move the price by $d_{i}$. A competitive trader would face a flat residual supply $\left(d_{i}=0\right)$. The slope $d_{i}$ increases, and the residual supply becomes less elastic, the steeper are the demand functions submitted by the other traders (i.e., the lower are $c_{i}$ and $c_{j}$ ).

As a consequence, this trader's information set $\left(s_{i}, p\right)$ is informationally equivalent to $\left(s_{i}, I_{i}\right)$. Note that only the intercept of the residual supply for a trader of type $i$ is informative about the signal of traders of type $j$. The bidder therefore chooses $x_{i}$ to maximize

$$
\mathbb{E}\left[\pi_{i} \mid s_{i}, p\right]=\left(\mathbb{E}\left[\theta_{i} \mid s_{i}, I_{i}\right]-I_{i}-d_{i} x_{i}\right) x_{i}-\lambda_{i} x_{i}^{2} / 2 .
$$

The first-order condition (FOC) is given by $\mathbb{E}\left[\theta_{i} \mid s_{i}, I_{i}\right]-I_{i}-2 d_{i} x_{i}-\lambda_{i} x_{i}=0$, or equivalently,

$$
X_{i}\left(s_{i}, p\right)=\left(\mathbb{E}\left[\theta_{i} \mid s_{i}, p\right]-p\right) /\left(d_{i}+\lambda_{i}\right) .
$$

\footnotetext{
${ }^{15}$ If there is no market clearing price the market shuts down and if there are multiple clearing prices the auctioneer choses the one that maximizes volume traded.

${ }^{16}$ We assume that $\left(n_{i}-1\right) c_{i}+n_{j} c_{j} \neq 0$.
} 
The second-order condition (SOC) that guarantees a maximum is $2 d_{i}+\lambda_{i}>0$, which implies that $d_{i}+\lambda_{i}>0$.

Using the expression for $I_{i}$ and assuming that $a_{j} \neq 0$, we can show that $\left(s_{i}, p\right)$ is informationally equivalent to $\left(s_{1}, s_{2}\right)$. Therefore, since $\mathbb{E}\left[\theta_{i} \mid s_{i}, p\right]=\mathbb{E}\left[\theta_{i} \mid s_{i}, I_{i}\right]$, it follows that

$$
\mathbb{E}\left[\theta_{i} \mid s_{i}, p\right]=\mathbb{E}\left[\theta_{i} \mid s_{1}, s_{2}\right]
$$

According to Gaussian distribution theory,

$$
\mathbb{E}\left[\theta_{i} \mid s_{i}, s_{j}\right]=\bar{\theta}_{i}+\Xi_{i}\left(s_{i}-\bar{\theta}_{i}\right)+\Psi_{i}\left(s_{j}-\bar{\theta}_{j}\right)
$$

where

$$
\Xi_{i}=\frac{1-\rho^{2}+\widehat{\sigma}_{\varepsilon_{j}}^{2}}{\left(1+\widehat{\sigma}_{\varepsilon_{i}}^{2}\right)\left(1+\widehat{\sigma}_{\varepsilon_{j}}^{2}\right)-\rho^{2}} \text { and } \Psi_{i}=\frac{\rho \widehat{\sigma}_{\varepsilon_{i}}^{2}}{\left(1+\widehat{\sigma}_{\varepsilon_{i}}^{2}\right)\left(1+\widehat{\sigma}_{\varepsilon_{j}}^{2}\right)-\rho^{2}}
$$

with $\widehat{\sigma}_{\varepsilon_{i}}^{2}=\sigma_{\varepsilon_{i}}^{2} / \sigma_{\theta}^{2}$ and $\widehat{\sigma}_{\varepsilon_{j}}^{2}=\sigma_{\varepsilon_{j}}^{2} / \sigma_{\theta}^{2}$. We remark that Equation (3) has the following implications.

1. The private signal $s_{i}$ is useful for predicting $\theta_{i}$ whenever $1-\rho^{2}+\widehat{\sigma}_{\varepsilon_{j}}^{2} \neq 0$, that is, when either the liquidation values are not perfectly correlated $(\rho \neq 1)$ or type- $j$ traders are imperfectly informed about $\theta_{j}\left(\sigma_{\varepsilon_{j}}^{2} \neq 0\right)$.

2. The private signal $s_{j}$ is useful for predicting $\theta_{i}$ whenever $\rho \widehat{\sigma}_{\varepsilon_{i}}^{2} \neq 0$, that is, when the private liquidation values are correlated $(\rho \neq 0)$ and type- $i$ traders are imperfectly informed about $\theta_{i}\left(\sigma_{\varepsilon_{i}}^{2} \neq 0\right)$.

Using the expression for demand (1) and the updating formulae we can derive a linear equilibrium by identifying coefficients. Our first proposition summarizes the result. It shows the relationship between $a_{i}$ and $c_{i}$ in equilibrium and also indicates that these coefficients are positive (see Lemma A1 and A2 in Appendix A for more details).

Proposition 1. Let $\rho<1$. If equilibrium exists, then it is unique and the demand function of a trader of type $i(i=1,2), X_{i}\left(s_{i}, p\right)=b_{i}+a_{i} s_{i}-c_{i} p$, is given by $X_{i}\left(s_{i}, p\right)=$ $\left(\mathbb{E}\left[\theta_{i} \mid s_{i}, p\right]-p\right) /\left(d_{i}+\lambda_{i}\right)$, with $d_{i}=1 /\left(\left(n_{i}-1\right) c_{i}+n_{j} c_{j}\right)$. In addition, we have that $a_{i}=$ $\Delta_{i} c_{i}>0$, where $\Delta_{i}=1 /\left(1+(1+\rho)^{-1} \widehat{\sigma}_{\varepsilon_{i}}^{2}\right), c_{i}=\left(1-\Lambda_{i}\right) /\left(d_{i}+\lambda_{i}\right)$, where $\Lambda_{i} \equiv \Psi_{i}\left(\frac{n_{i} c_{i}}{n_{j} c_{j}}+1\right) / \Delta_{j}$, and the ratio $c_{1} / c_{2}$ is the unique positive solution of a cubic polynomial.

The term $\Lambda_{i}$ in the price coefficient in the equilibrium demand $c_{i}$ is the information-sensitivity weight of the price (i.e., the coefficient of the price in $\mathbb{E}\left[\theta_{i} \mid s_{i}, p\right]$ ). The larger $\Lambda_{i}$, the lower the price coefficient in demand will be (lower $c_{i}$ ). From the perspective of a bidder in group $i$ a high price conveys the news that $s_{j}$ is high and, therefore, that the value $\theta_{i}$ will tend to be 
high. If the price is more informative about $\theta_{i}$, then a bidder in group $i$ is more cautious and submits then a steeper schedule. Furthermore, $\Lambda_{i}$ vanishes when $\Psi_{i}=0$, that is, when either the valuations are uncorrelated $(\rho=0)$ or the private signal $s_{i}$ is perfectly informative $\left(\sigma_{\varepsilon_{i}}^{2}=0\right)$, since in those cases the price conveys no additional information to a trader of type $i$. In this case $c_{i}=1 /\left(d_{i}+\lambda_{i}\right)$ and since $d_{i}=1 /\left(\left(n_{i}-1\right) c_{i}+n_{j} c_{j}\right)$ we observe, therefore, a basic strategic complementarity in the slopes of the demands submitted by the traders. According to this strategic effect if the rivals of a trader of type $i$, say traders of type $j$, bid a steeper demand function with a lower $c_{j}$, then the slope of the (inverse) residual supply $d_{i}$ for this trader increases and so he also has an incentive to bid a steep demand function (lower $c_{i}$ ). However, if $\Psi_{i}>0$ then there is also an inference effect from the information conveyed by the price. A lower $c_{j}$ increases $\Lambda_{i}$ and also will tend to depress $c_{i}$. This is so because a lower reaction to the price and signal by rivals induces a trader of type $i$ to give a higher weight to the price in the estimation of his value and, hence, the magnitude of the inference effect raises.

Since $a_{i}>0$ and $c_{i}>0$, for $i=1,2$, it follows that in equilibrium the higher the value of the trader's observed private signal (or the lower the price), the higher the quantity she will demand. When $\sigma_{\varepsilon_{i}}^{2}>0$ we have $a_{i}<c_{i}$, since $\Delta_{i}<1$ in this case; when $\sigma_{\varepsilon_{i}}^{2}=0$, we have $\Delta_{i}=1$ and $a_{i}=c_{i}$. Observe that we can write the demand as $X_{i}\left(s_{i}, p\right)=b_{i}+c_{i}\left(\Delta_{i} s_{i}-p\right)$.

Because $p$ is a linear function of $s_{1}$ and $s_{2}$, for $i=1,2$ we have $\mathbb{E}\left[\theta_{i} \mid s_{i}, p\right]=\mathbb{E}\left[\theta_{i} \mid s_{1}, s_{2}\right]$ (i.e., Equation (2) holds). The equilibrium price is therefore privately revealing, in other words, the private signal and the price enable a type- $i$ trader to learn as much as about $\theta_{i}$ if she had access to all the information available in the market, $\left(s_{1}, s_{2}\right){ }^{17}$

If $\rho=0$ or if both signals, $i=1,2$, are perfectly informative $\left(\sigma_{\varepsilon_{i}}^{2}=0\right.$ ), or useless (or $\sigma_{\varepsilon_{i}}^{2}=$ $\infty)$, then bidders do not learn about $\theta_{i}$ from prices. Hence, $\mathbb{E}\left[\theta_{i} \mid s_{i}\right]=\mathbb{E}\left[\theta_{i} \mid s_{i}, p\right]=\mathbb{E}\left[\theta_{i} \mid s_{1}, s_{2}\right]$ for $i=1,2$. The demand functions are given by

$$
X_{i}\left(s_{i}, p\right)=\left(\mathbb{E}\left[\theta_{i} \mid s_{i}\right]-p\right) /\left(d_{i}+\lambda_{i}\right), i=1,2 .
$$

Hence, $c_{i}=1 /\left(d_{i}+\lambda_{i}\right)$ and in this case, the equilibrium coincides with the full-information equilibrium (denoted by superscript $f$ ). ${ }^{18}$ Furthermore, when $\rho=0, d_{i}$ is independent of $\sigma_{\varepsilon_{i}}^{2}$, $i=1,2$; and when $\sigma_{\varepsilon_{i}}^{2}=0$ or $\sigma_{\varepsilon_{i}}^{2}=\infty$, for $i=1,2, d_{i}$ is independent of $\rho$.

Our next proposition summarizes when equilibrium exists. If an equilibrium does exist, then Proposition 1 implies that it is unique.

\footnotetext{
${ }^{17}$ This would not be the case if there were more than two groups or if the traders in each group were to receive idiosyncratic signals. In this case there would appear an information externality which would induce additional ineffiencies in the market. The situation would be similar to the case of a noisy equilibrium (e.g. Vives 2017)

${ }^{18}$ In the full (shared) information setup, traders can access $\left(s_{1}, s_{2}\right)$. In this framework the price does not provide any useful information.
} 
Proposition 2. Equilibrium exists iff $c_{i}>0, i=1,2$.

Complete information. When $\rho=0$, or $\sigma_{\varepsilon_{i}}^{2}=0$, or $\sigma_{\varepsilon_{i}}^{2}=\infty, i=1,2$, equilibrium exists iff $n_{1}+n_{2} \geq 3$.

Incomplete information. When $\rho \sigma_{\varepsilon_{i}}^{2}>0$ and $\sigma_{\varepsilon_{j}}^{2} \geq 0, j \neq i$, and $\rho<1$, we find a necessary and sufficient condition for $c_{i}>0, i=1,2$ (see Proposition $2 A$ in Appendix A). It follows that equilibrium exists if: (i) $n_{1}, n_{2}$ are large enough; (ii) given $n_{i}, n_{j}$ is large enough and $\rho$ low enough, for $i, j=1,2, j \neq i$, (iii) $\sigma_{\varepsilon_{j}}^{2}=0$ and $n_{j} \geq 2$.

Remark 1. Equilibrium does not exist for $\rho$ close to 1 and low $n_{i}$. This is so because in those cases the market power of traders explodes and the demand schedules would become vertical (with $c_{i} \rightarrow 0, i=1,2$ ). As $\rho$ increases the informational component of the price is more important and traders submit steeper demands (see Proposition 3 below). Neither does an equilibrium exist when $\rho=1$. If the price reveals a sufficient statistic for the common valuation, then no trader has an incentive to place any weight on her signal. But if traders put no weight on signals, then the price contains no information about the common valuation. This conundrum is related to the Grossman-Stiglitz (1980) paradox. In fact, $\rho<1$ and $n_{1}+n_{2} \geq 3$ are necessary conditions for the existence of equilibrium with incomplete information (in line with Kyle 1989; Vives 2011). ${ }^{19}$

To develop a better understanding of the equilibrium and the condition that guarantees its existence, we consider three particular cases of the model: a monopsony competing with a fringe, symmetric groups, and an informed and an uninformed group.

\section{Monopsony competing with fringe}

Corollary 1. For $n_{2}=1$ the equilibrium exists if $1-\rho^{2}>(2 \rho-1) \widehat{\sigma}_{\varepsilon_{1}}^{2}$ and $n_{1}>$ $\bar{n}_{1}\left(\rho, \widehat{\sigma}_{\varepsilon_{1}}^{2}, \widehat{\sigma}_{\varepsilon_{2}}^{2}\right)$, where $\bar{n}_{1}$ increases with $\rho, \widehat{\sigma}_{\varepsilon_{1}}^{2}$, and $\widehat{\sigma}_{\varepsilon_{2}}^{2}$. If, also, $\lambda_{2}=0$ and $\sigma_{\varepsilon_{2}}^{2}=0$, then $\bar{n}_{1}\left(\rho, \widehat{\sigma}_{\varepsilon_{1}}^{2}, 0\right)=1+\rho \widehat{\sigma}_{\varepsilon_{1}}^{2} /\left(1-\rho^{2}-(2 \rho-1) \widehat{\sigma}_{\varepsilon_{1}}^{2}\right)$ and $x_{2}=c_{2}\left(\theta_{2}-p\right)$, with $c_{2}=n_{1} c_{1}$.

An equilibrium with linear demand functions exists provided there is a sufficiently competitive trading environment ( $n_{1}$ high enough, and with the threshold $\bar{n}_{1}$ increasing with $\rho$ and $\widehat{\sigma}_{\varepsilon_{i}}^{2}$ $(i=1,2)$, which raise the informational component of the price). In the particular case where $\lambda_{2}=0$ and $\sigma_{\varepsilon_{2}}^{2}=0$, expressions for the equilibrium coefficients can be characterized explicitly (see Appendix A). From the expressions for $c_{i}(i=1,2)$ it follows that, if $n_{1}=\bar{n}_{1}$, then the equilibrium cannot exist because in this case the demand functions would be completely inelastic $\left(c_{i}=0, i=1,2\right)$.

\footnotetext{
${ }^{19} \mathrm{Du}$ and Zhu (2016) consider ex post nonlinear equilibria in a bilateral divisible double auction and show that with more than three symmetric traders there are no nonlinear equilibria in the class of smooth demands downward sloping in price and upward sloping in signals.
} 


\section{Symmetric groups}

Consider the following symmetric case: $n_{i}=n, \lambda_{i}=\lambda$, and $\sigma_{\varepsilon_{i}}^{2}=\sigma_{\varepsilon}^{2}, i=1,2$. We find that equilibrium exists iff $n>1+\rho \widehat{\sigma}_{\varepsilon}^{2} /\left((1-\rho)\left(1+\rho+\widehat{\sigma}_{\varepsilon}^{2}\right)\right)$, where $\widehat{\sigma}_{\varepsilon}^{2}=\sigma_{\varepsilon}^{2} / \sigma_{\theta}^{2}$. Therefore, the equilibrium's existence is guaranteed provided either that $n$ is high enough or that $\rho$ or $\widehat{\sigma}_{\varepsilon}^{2}$ is low enough.

Vives (2011) also analyzes divisible good auctions with symmetric bidders, but in his model the bidders receive different private signals. The condition that guarantees existence of an equilibrium in Vives' setup is $n>1+n \rho \widehat{\sigma}_{\varepsilon}^{2} /\left((1-\rho)\left(1+(2 n-1) \rho+\widehat{\sigma}_{\varepsilon}^{2}\right)\right)$. Direct computation yields that the condition derived in the model of Vives is more stringent than the condition derived in our setup. The reason is that, in Vives (2011), the degree of asymmetry in information (and induced market power) is greater because each of the $2 n$ traders receives a private signal.

\section{Informed and uninformed groups}

Consider the case where group 1 is uninformed (with no signal) while group 2 is informed $\left(\sigma_{\varepsilon_{2}}^{2}<\infty\right)$. This is akin to the information structure considered by Grossman and Stiglitz (1980). Then equilibrium exists if and only if $\rho<\frac{n_{2}\left(n_{1}+n_{2}-2\right)}{2 n_{1}+n_{2}-2}$, which again holds for $\rho$ small (the threshold is increasing in $n_{2}$ and decreasing in $n_{1}$ for $\left.n_{2}>2\right)$. We have that $a_{2}=\left(1+\widehat{\sigma}_{\varepsilon_{2}}^{2}\right)^{-1} c_{2} \cdot{ }^{20}$ This expression is different from the case in which group 1 is informed $\left(\sigma_{\varepsilon_{1}}^{2}<\infty\right)$ since then

$a_{2}=\left(1+\widehat{\sigma}_{\varepsilon_{2}}^{2} /(1+\rho)\right)^{-1} c_{2}$. Therefore, the equilibrium that we obtain here is not the limit of the general case when $\sigma_{\varepsilon_{1}}^{2} \rightarrow \infty$ for $\rho>0$. This is so, since when $\rho>0$, no matter how large $\sigma_{\varepsilon_{1}}^{2}$ is, but finite, there is learning from the price by the two groups. By contrast, when group 1 does not receive any private signal, there is no learning from the price by group 2. Furthermore, in this last case, we have that $c_{1}$ and $c_{2}\left(d_{1}\right.$ and $\left.d_{2}\right)$ are independent of $\sigma_{\varepsilon_{2}}^{2}$, and $b_{2}$ is independent of $\bar{\theta}_{1}$ and $Q$.

\subsection{Comparative statics}

We start by considering how the model's underlying parameters affect the equilibrium and, in particular, price impact (Proposition 3). We then explore how the equilibrium is affected when there are two distinct groups of traders, that is, a strong group and a weak group (Corollary 2).

Proposition 3. Let $\rho \sigma_{\varepsilon_{1}}^{2} \sigma_{\varepsilon_{2}}^{2}>0$. Then, for $i=1,2, i \neq j$, the following statements hold.

(i) An increase in $\bar{\theta}_{i}$ or $Q$, or a decrease in $\bar{\theta}_{j}$, raises the demand intercept $b_{i}$.

(ii) An increase in $\lambda_{i}, \lambda_{j}, \sigma_{\varepsilon_{i}}^{2}, \sigma_{\varepsilon_{j}}^{2}$, or $\rho$ makes demand less responsive to private signals and prices (lower $a_{i}$ and $c_{i}$ ) and increases price impact $\left(d_{i}\right)$.

\footnotetext{
${ }^{20}$ In the case where $\sigma_{\varepsilon_{1}}^{2}<\infty$ and $\sigma_{\varepsilon_{2}}^{2}=0$, then $b_{2}=0$ and $a_{1}=c_{1}$.
} 
(iii) If $\sigma_{\varepsilon_{i}}^{2}$ and/or $\lambda_{i}$ increase, then $d_{i} / d_{j}$ decreases.

(iv) If $n_{i}$ and/or $n_{j}$ increase, then $d_{i}$ decreases.

Remark 2. In the complete information setting $\left(\rho=0\right.$ or when $\sigma_{\varepsilon_{i}}^{2}=0$, or $\sigma_{\varepsilon_{i}}^{2}=\infty$, $i=1,2)$ prices do not convey information, $d_{i}^{f}$ and $d_{j}^{f}, j \neq i$, are independent of $\sigma_{\varepsilon_{i}}^{2}$; and comparative statics of $d_{i}^{f}, d_{j}^{f}$ on $\lambda_{i}, n_{i}$ hold as in the proposition. ${ }^{21}$ Indeed, if $\rho=0$, then: (a) both $c_{i}$ and $d_{i}$ (as well as $c_{j}$ and $a_{j}, j \neq i$ ) are independent of $\sigma_{\varepsilon_{i}}^{2}$; (b) $a_{i}$ decreases with $\sigma_{\varepsilon_{i}}^{2}$; and (c) $b_{i}$ is independent of both $Q$ and $\bar{\theta}_{j}$. If $\sigma_{\varepsilon_{i}}^{2}=0$ for $i=1,2$, then $b_{i}=0$, and $c_{i}, c_{j}, a_{i}, a_{j}, d_{i}$, and $d_{j}, i=1,2, j \neq i$, are independent of $\rho$. That is, for the information parameters to matter for price impact, it is necessary that prices convey information. Proposition 3(ii) implies that, if $\rho \sigma_{\varepsilon_{1}}^{2} \sigma_{\varepsilon_{2}}^{2}>0$, then $d_{i}^{f}<d_{i}, i=1,2$. Thus, asymmetric information increases the price impact of traders in both groups beyond the full-information level.

Remark 3. When groups are symmetric then results (ii) and (iv) hold when $\lambda_{i}=\lambda_{j}, \sigma_{\varepsilon_{i}}^{2}=$ $\sigma_{\varepsilon_{j}}^{2}$, and $n_{i}=n_{j}$ move together.

The only equilibrium coefficient affected by the quantity offered in the auction $(Q)$ and by the prior mean of the valuations $\left(\bar{\theta}_{i}\right.$ and $\left.\bar{\theta}_{j}\right)$ is $b_{i}$. Proposition 3(i) indicates that if $Q$ increases, then all the bidders will increase their demand (higher $b_{1}$ and $b_{2}$ ). Moreover, if the prior mean of the valuation of group $i$ increases, then the bidders in this group will demand a greater quantity of the risky asset (higher $b_{i}$ ). Then the intercept $I_{j}$ of the inverse residual supply for the group $j$ bidder rises in response to a higher $\bar{\theta}_{i}$. That reaction leads the traders in group $j$ to reduce their demand for the risky asset (and lower $b_{j}$ ). We see thus here a strategic substitutability in demand intercepts.

All these results provide testable predictions. Furthermore, if we have estimates of transactions costs, precision of the signals and correlation of values we can predict how changes in these parameters lead to changes in the slopes of submitted demands and the price impact of the two groups.

Part (ii) of Proposition 3 shows how the response to private information and price varies with several parameters. If the transaction costs for a bidder of type $i, \lambda_{i}$, increase, then the bidder sets lower $a_{i}$ and $c_{i}$. Moreover, any increase in a group's transaction costs also affects the behavior of traders in the other group. If $\lambda_{i}$ increases, then $a_{i}$ and $c_{i}$ decrease, in which case the slope of the inverse residual supply for group $j$ increases (higher $d_{j}$ ) as well as the weight on the price $\Lambda_{j}$ in the estimation of $\theta_{j}$. That is, both the strategic and the inference effects work in the same direction. This change leads group- $j$ traders to reduce their demand sensitivity to signals and prices (lower $a_{j}$ and $c_{j}$ ). We can therefore see how an increase in the transaction

\footnotetext{
${ }^{21}$ Akgün (2004) considers a complete information model and shows (in our notation) that an increase in $\lambda_{i}$ reduces $c_{i}$ and $c_{j}$.
} 
costs for group- $i$ traders (say, a deterioration of their collateral in liquidity auctions that raises $\lambda_{i}$ ) leads not only to steeper demands for bidders in group $i$ but also, as a reaction, to steeper demands for group- $j$ traders.

We analyze how the response to private information and price varies with a change in the precision of private signals. If the private signal of type- $i$ bidders is less precise (higher $\sigma_{\varepsilon_{i}}^{2}$ ), then their demand is less sensitive to private information and prices. A private signal of reduced precision also gives the type- $i$ bidder more incentive to consider prices when predicting $\theta_{i}$ (higher $\left.\Lambda_{i}\right),{ }^{22}$ which leads in turn to this bidder having a steeper demand function (lower $c_{i}$ ). The same can be said for a bidder of type $j$ because of strategic complementarity in the slopes of demand functions (the decrease in $c_{i}$ leads to increased $d_{j}$ and to lower $a_{j}$ and $c_{j}$ in turn). ${ }^{23}$ This result (in the supply competition model interpretation) may help explain why, in the Texas balancing market, small firms use steeper supply functions than predicted by theory (Hortaçsu and Puller 2008). Indeed, smaller firms may receive lower-quality signals owing to economies of scale in information gathering.

We also find that the more highly the valuations are correlated (higher $\rho$ ), the less is trader responsiveness to private signals (lower $a_{i}, i=1,2$ ) and the steeper are inverse demand functions (lower $c_{i}, i=1,2$ ). We can explain these results by recalling that, when the valuations are correlated $(\rho>0)$, a type- $i$ trader learns about $\theta_{i}$ from prices. In fact, the informationsensitivity weight on the price $\left(\Lambda_{i}, i=1,2\right)$ is higher when $\rho$ is larger, ${ }^{24}$ in which case demand is less sensitive to private information. The rationale for the relationship between the correlation coefficient $(\rho)$ and the slopes of demand functions is as follows. An increment in the price of the risky asset makes an agent more optimistic about her valuation, which leads to less of a reduction in demand quantity than in the case of uncorrelated valuations. ${ }^{25}$

Cassola et al. (2013) show how distressed bidders after the August 2007 shock suffered a large decline in the valuation of their collateral in the interbank market, which in terms of our model shows up in an increased $\lambda_{i}$. Those banks had also an increase in the valuation for liquidity (which in our model shows up as an increased $\left.\bar{\theta}_{i}\right) .{ }^{26}$ In Cassola et al. (2013) it is assumed that

\footnotetext{
${ }^{22}$ It is easy to check that $\Psi_{i} / \Delta_{j}, i \neq j, i=1,2$, is increasing in $\sigma_{\varepsilon_{i}}^{2}$.

${ }^{23} \mathrm{An}$ increased $\sigma_{\varepsilon_{i}}^{2}$ leads to lower inference component in the price for a bidder of type $j, \Lambda_{j}$, but this effect is overpowered by the increase in $d_{j}$ in its influence on $c_{j}$.

${ }^{24}$ It is easy to check that $\Psi_{i} / \Delta_{j}, i \neq j, i=1,2$, is increasing in $\rho$.

${ }^{25} \mathrm{~A}$ high price conveys the good news that the private signal received by other group's traders is high. When valuations are positively correlated, a bidder infers from the high private signal of the other group that her own valuation is high.

${ }^{26}$ The marginal valuation of a bidder of type $i$ is $\theta_{i}-\lambda_{i} x_{i}$. This is akin to the marginal valuation in Figure 4 in Cassola et al. (2013). There a decreased collateralized borrowing capacity of a bidder $(K)$ will make the slope of the marginal valuation steeper.
} 
the private valuations of the traders are independent since the common component is known. This means that there are no information effects. However, if the common value component is not known, as it plausible to believe, and if in the crisis the signals of the groups become noisier, in particular for those of the group hit by the shock, and the correlation of valuations increases, then all these effects reinforce the steepening of the demand schedules (as found in Cassola et al. (2013)).

Figure 1 illustrates the case of initially identical groups that become differentiated after a shock induces a higher $\lambda_{1}$, noise in the signal for group $1\left(\sigma_{\varepsilon_{1}}^{2}\right)$ and correlation $\rho$ as well as the groups' willingness to pay for liquidity (both $\bar{\theta}_{1}$ and $\bar{\theta}_{2}$, which affect the intercepts of the demand functions). This corresponds to the case of a group of banks being hit in the crisis and the quality of their information deteriorating (or their perceived uncertainty increasing) as well as the correlation of valuations raising. Note that the expected marginal valuation of liquidity for the safe group need not change a lot (consistent with findings of Cassola et al. (2013)).

\section{[Figure 1 here]}

Proposition 3(iii) states that any increase in the signal's noise or in group $i$ 's transaction costs has the effect of reducing its relative price impact, since then the ratio $d_{i} / d_{j}(i \neq j)$ decreases. Finally, part (iv) formalizes the anticipated result that an increase in the number of auction participants (higher $n_{i}$ or $n_{j}$ ) reduces the price impact of traders in both groups.

Corollary 2 (Strong and weak groups). Suppose that $\sigma_{\varepsilon_{1}}^{2} \geq \sigma_{\varepsilon_{2}}^{2}, \lambda_{1} \geq \lambda_{2}$, and $n_{1} \geq n_{2}$, and suppose that at least one of these inequalities is strict. Then, in equilibrium, the following statements hold.

(i) The stronger group (here, group 2) reacts more both to private information and to prices $\left(a_{1}<a_{2}, c_{1}<c_{2}\right)$ and has more price impact $\left(d_{1}<d_{2}\right)$ than does the weaker group.

(ii) The value of the difference $d_{1}+\lambda_{1}-\left(d_{2}+\lambda_{2}\right)$ is, in general, ambiguous:

- $d_{1}+\lambda_{1}>d_{2}+\lambda_{2}$ whenever the differences between groups mainly stem from the transaction costs and $\lambda_{1} / \lambda_{2}$ is high enough (with complete information, $d_{1}+\lambda_{1}>d_{2}+\lambda_{2}$ iff $\lambda_{1}>\lambda_{2}$ );

- $d_{1}+\lambda_{1}<d_{2}+\lambda_{2}$ with $\lambda_{1}>\lambda_{2}$ whenever $\rho$ is large.

Part (i) of this corollary shows that if a group of traders is less informed, has higher transaction costs, and is more numerous, then it reacts less both to private signals and to prices. Observe in particular that group-1 traders, having less precise private information, rely more on the price for information (higher $\left.\Lambda_{1}\right)$; as a result, their overall price response $\left(c_{1}=\left(1-\Lambda_{1}\right) /\left(d_{1}+\lambda_{1}\right)\right)$ is smaller. Similarly, group-1 traders, for whom $n_{1}$ is larger, put more information-sensitivity weight on the price (which depends more strongly on $s_{1}$ ). 
The precise conditions for Corollary 2(ii), which is useful for comparing allocations across groups, are given in Corollary 2A(ii) in Appendix A. When we have that $d_{1}+\lambda_{1}<d_{2}+\lambda_{2}$ with $\lambda_{1}>\lambda_{2}$ it means that traders in the group with lower transaction costs are more cautious in order to diminish price impact.

\subsection{Equilibrium quantities}

Let $t_{i}=\mathbb{E}\left[\theta_{i} \mid s_{1}, s_{2}\right], i=1,2$, be the predicted values with full information $\left(s_{1}, s_{2}\right)$. After some algebra, it follows that equilibrium quantities are functions of the vector of predicted values $t=\left(t_{1}, t_{2}\right)$ :

$$
\begin{aligned}
& \qquad \begin{aligned}
x_{i}(t) & =\underbrace{\frac{n_{j}\left(t_{i}-t_{j}\right)}{u}}_{x_{i}^{V}(t)}+\underbrace{\frac{d_{j}+\lambda_{j}}{u} Q}_{x_{i}^{C}(t)}, i=1,2, j \neq i, \\
\text { where } u & =n_{i}\left(d_{j}+\lambda_{j}\right)+n_{j}\left(d_{i}+\lambda_{i}\right) .
\end{aligned}
\end{aligned}
$$

Observe that, according to these expressions, the equilibrium quantities can be decomposed into two terms: a valuation trading term and a clearing trading term, which we denote by (respectively) $x_{i}^{V}(t)$ and $x_{i}^{C}(t)$ for group $i, i=1,2$. With regard to the information trading term, it vanishes when $t_{1}=t_{2}$, but has a positive (resp. negative) value for the group with the higher (resp. lower) value of $t_{i}$. Higher "total" transaction costs $\left(d_{i}+\lambda_{i}\right)$ lower the response to valuation differences $t_{i}-t_{j}$. Moreover, $n_{1} x_{1}^{V}(t)+n_{2} x_{2}^{V}(t)=0$. As for the clearing trading term, we remark that it vanishes when $Q=0$; otherwise, it is positive for both groups yet lower (resp. higher) for the group with higher (resp. lower) $d_{i}+\lambda_{i}$. In addition, $n_{1} x_{1}^{C}(t)+n_{2} x_{2}^{C}(t)=Q$.

Taking expectations in Equation (4), we have

$$
\mathbb{E}\left[x_{1}(t)\right]-\mathbb{E}\left[x_{2}(t)\right]=\frac{\left(n_{1}+n_{2}\right)\left(\bar{\theta}_{1}-\bar{\theta}_{2}\right)+\left(d_{2}+\lambda_{2}-\left(d_{1}+\lambda_{1}\right)\right) Q}{n_{1}\left(d_{2}+\lambda_{2}\right)+n_{2}\left(d_{1}+\lambda_{1}\right)} .
$$

Group 1 trades more when it values the asset more highly $\left(\bar{\theta}_{1}>\bar{\theta}_{2}\right)$ and when its traders are less cautious $\left(d_{2}+\lambda_{2}>d_{1}+\lambda_{1}\right)$ than group 2. By combining Corollary 2 with the equation just displayed, we obtain the following remarks.

Remark 4. If $Q$ is low enough, then $\mathbb{E}\left[x_{1}(t)\right]>\mathbb{E}\left[x_{2}(t)\right]$ whenever $\bar{\theta}_{1}>\bar{\theta}_{2}$. In contrast, if $Q$ is high enough, then $\mathbb{E}\left[x_{1}(t)\right]>\mathbb{E}\left[x_{2}(t)\right]$ whenever $d_{2}+\lambda_{2}>d_{1}+\lambda_{1}$. Under the assumptions of Corollary 2, this latter inequality is satisfied if (34) holds or if $\lambda_{1} / \lambda_{2}$ is sufficiently low.

Remark 5. When $Q=0$ (i.e., the so-called double auction case), then $\mathbb{E}\left[x_{2}(t)\right]<0<$ $\mathbb{E}\left[x_{1}(t)\right]$ iff $\bar{\theta}_{1}>\bar{\theta}_{2}$. Then group 1 consists of buyers and group 2 of sellers. 


\section{Welfare analysis}

We identify factors that affect, in equilibrium, the expected price and revenue in the auction in Subsection 4.1 (see Appendix B for an analysis of bid shading), the equilibrium and efficient allocations in Subsection 4.2, analyze deadweight losses in Subsection 4.3, and market integration in Subsection 4.4 .

\subsection{Expected price and revenue}

Let $\tilde{t}=\left(n_{1} t_{1}+n_{2} t_{2}\right) /\left(n_{1}+n_{2}\right)$. From the demand of bidders it follows that $p(t)=t_{i}-\left(d_{i}+\right.$ $\left.\lambda_{i}\right) x_{i}(t), i=1,2$. Therefore,

$$
p(t)=\tilde{t}-\left(\left(d_{1}+\lambda_{1}\right) n_{1} x_{1}(t)+\left(d_{2}+\lambda_{2}\right) n_{2} x_{2}(t)\right) /\left(n_{1}+n_{2}\right) .
$$

From the above expression we can derive the expected price:

$$
\mathbb{E}[p(t)]=\left(\frac{n_{1}}{d_{1}+\lambda_{1}} \bar{\theta}_{1}+\frac{n_{2}}{d_{2}+\lambda_{2}} \bar{\theta}_{2}-Q\right) /\left(\frac{n_{1}}{d_{1}+\lambda_{1}}+\frac{n_{2}}{d_{2}+\lambda_{2}}\right) .
$$

It is worth noting that, in the double auction case $(Q=0), \mathbb{E}[p]$ is a convex combination of $\bar{\theta}_{1}$ and $\bar{\theta}_{2}$. Also, for symmetric groups (except possibly with respect to the means) we have $\mathbb{E}[p]=\left(\bar{\theta}_{1}+\bar{\theta}_{2}\right) / 2$.

Proposition 4. Let $\rho \sigma_{\varepsilon_{1}}^{2} \sigma_{\varepsilon_{2}}^{2}>0$. In equilibrium, the following statements hold.

(i) If $\bar{\theta}_{1}=\bar{\theta}_{2}$, then the expected price is increasing in $n_{i}$ but is decreasing in $\lambda_{i}$, $\sigma_{\varepsilon_{i}}^{2}$, and $\rho$, $i=1,2$. Otherwise, if $\left|\bar{\theta}_{1}-\bar{\theta}_{2}\right|$ is large enough, then these results need not hold.

(ii) The seller's expected revenue $\mathbb{E}[p] Q$ :

- increases with $\bar{\theta}_{i}$ for $i=1,2$;

- is maximum when $Q=\left(\frac{n_{1}}{d_{1}+\lambda_{1}} \bar{\theta}_{1}+\frac{n_{2}}{d_{2}+\lambda_{2}} \bar{\theta}_{2}\right) / 2$, which increases with $n_{i}$ and $\bar{\theta}_{i}$ and decreases with $\rho, \lambda_{i}$ and $\sigma_{\varepsilon_{i}}^{2}, i=1,2$.

Corollary 3. The expected revenue is between (a) the larger expected revenue of the auction in which both groups are ex ante identical with a large number of bidders (each group with $\left.\max \left\{n_{1}, n_{2}\right\}\right)$, high expected valuation $\left(\max \left\{\bar{\theta}_{1}, \bar{\theta}_{2}\right\}\right)$, low transaction costs $\left(\min \left\{\lambda_{1}, \lambda_{2}\right\}\right)$ and precise signals $\left(\min \left\{\sigma_{\varepsilon_{1}}^{2}, \sigma_{\varepsilon_{2}}^{2}\right\}\right)$ and $(\mathrm{b})$ the smaller expected revenue of the auction in which both groups are ex ante identical but with the opposite characteristics (i.e., $\min \left\{n_{1}, n_{2}\right\}, \min \left\{\bar{\theta}_{1}, \bar{\theta}_{2}\right\}$, $\max \left\{\lambda_{1}, \lambda_{2}\right\}$, and $\left.\max \left\{\sigma_{\varepsilon_{1}}^{2}, \sigma_{\varepsilon_{2}}^{2}\right\}\right)$.

Remark 6. If $\rho=0$, then $\mathbb{E}[p]$ is independent of $\sigma_{\varepsilon_{i}}^{2}(i=1,2)$, and if $\sigma_{\varepsilon_{i}}^{2}=0, i=1,2$, then $\mathbb{E}[p]$ is independent of $\rho$. The reason is that in both cases, $d_{i}$ is independent of $\sigma_{\varepsilon_{i}}^{2}$ and $\rho$. If $\sigma_{\varepsilon_{1}}^{2}=\infty$, then $\mathbb{E}[p]$ is independent of $\sigma_{\varepsilon_{2}}^{2}$. 
Proposition 4 indicates that the relationship between expected price (on the one hand) and $\lambda_{i}, \sigma_{\varepsilon_{i}}^{2}$, and $\rho, i=1,2$ (on the other hand) is potentially ambiguous. For example, if $\bar{\theta}_{2}-\bar{\theta}_{1}$ is high enough, then $\mathbb{E}[p]$ is decreasing in $n_{1}$; yet, if $\bar{\theta}_{1}=\bar{\theta}_{2}$, then the derived results are in line with those in the symmetric case, where $\mathbb{E}[p]=\bar{\theta}-(d+\lambda) Q / 2 n$ (see Vives 2010, Prop. 2).

We should like to understand how ex ante differences among bidders affect the seller's expected revenue. Suppose that group 2 is our strong group; it has lower transaction costs $\left(\lambda_{2}<\lambda_{1}\right)$, is less numerous $\left(n_{2}<n_{1}\right)$, and is better informed $\left(\sigma_{\varepsilon_{2}}^{2}<\sigma_{\varepsilon_{1}}^{2}\right)$. If this group values the asset less, $\bar{\theta}_{2}<\bar{\theta}_{1}$ (resp., values it more, $\bar{\theta}_{2}>\bar{\theta}_{1}$ ), then expected revenue is lower (resp., higher) than in the case where $\bar{\theta}_{1}=\bar{\theta}_{2}$. If $\bar{\theta}_{1} \approx \bar{\theta}_{2}$, then Proposition 4(i) suggests that group 2's relatively small size $\left(n_{2}<n_{1}\right)$ reduces the seller's expected revenue, although both its relatively low transaction costs $\left(\lambda_{2}<\lambda_{1}\right)$ and its relatively precise signals $\left(\sigma_{\varepsilon_{2}}^{2}<\sigma_{\varepsilon_{1}}^{2}\right)$ have the opposite effect. So in general, the ex ante differences between the two groups have an ambiguous effect on the seller's expected revenue. Nonetheless, part (ii) of Proposition 4 directly follows from part (i).

\subsection{Characterizing the equilibrium and efficient allocations}

Recall that $t_{i}=\mathbb{E}\left[\theta_{i} \mid s_{1}, s_{2}\right], i=1,2$, that is, the predicted values with full information $\left(s_{1}, s_{2}\right)$ and $t=\left(t_{1}, t_{2}\right)$. The strategies in the equilibrium induce outcomes as functions of the realized vector of predicted values $t$ and are given in Equation (4). One can easily show that the equilibrium outcome solves the following distorted benefit maximization program: ${ }^{27}$

$$
\begin{gathered}
\max _{x_{1}, x_{2}} \mathbb{E}\left[n_{1}\left(\theta_{1} x_{1}-\left(d_{1}+\lambda_{1}\right) x_{1}^{2} / 2\right)+n_{2}\left(\theta_{2} x_{2}-\left(d_{2}+\lambda_{2}\right) x_{2}^{2} / 2\right) \mid t\right] \\
\text { s.t. } n_{1} x_{1}+n_{2} x_{2}=Q,
\end{gathered}
$$

where $d_{1}$ and $d_{2}$ are the equilibrium parameters. The efficient allocation would obtain if we set $d_{1}=d_{2}=0$, which corresponds to a price-taking equilibrium (denoted by superscript $o$ ). ${ }^{28}$ The equilibrium strategy of a type- $i$ bidder $(i=1,2)$ will be of the form $X_{i}^{o}\left(s_{i}, p\right)=b_{i}^{o}+a_{i}^{o} s_{1}-c_{i}^{o} p$, $i=1,2$, and is derived by maximizing the following program:

$$
\max _{x_{i}}\left(\mathbb{E}\left[\theta_{i} \mid s_{i}, p\right]-p\right) x_{i}-\lambda_{i} x_{i}^{2} / 2,
$$

while taking prices as given. The FOC of this optimization problem yields

$$
\mathbb{E}\left[\theta_{i} \mid s_{i}, p\right]-p-\lambda_{i} x_{i}=0
$$

\footnotetext{
${ }^{27}$ See Lemma A3 in Appendix A.

${ }^{28}$ The efficient allocation maximizes the expected total surplus. Here the revenue collected is just a transfer from the bidders to the auctioneer and washes out. If the social objective is just the surplus of the bidders or the revenue of the auctioneer, the objective function should be modified accordingly.
} 
After identifying coefficients and solving the corresponding system of equations, we find that there exists a unique equilibrium in this setup.

Proposition 5. Let $Q=\left(n_{1}+n_{2}\right) q$ and let $\mu_{i}=n_{i} /\left(n_{1}+n_{2}\right)$ for $i=1,2$. Then there exists a unique price-taking equilibrium, and the equilibrium coefficients of the demand function are given by

$$
\begin{aligned}
b_{i}^{o} & =\frac{\widehat{\sigma}_{\varepsilon_{i}}^{2}\left(\mu_{j}\left(\bar{\theta}_{i}-\rho \bar{\theta}_{j}\right)+\rho \lambda_{j} q\right)}{\mu_{i} \rho \widehat{\sigma}_{\varepsilon_{i}}^{2} \lambda_{j}+\mu_{j}\left(1-\rho^{2}+\widehat{\sigma}_{\varepsilon_{i}}^{2}\right) \lambda_{i}}, a_{i}^{o}=\frac{\mu_{j}\left(1-\rho^{2}\right)}{\mu_{i} \rho \widehat{\sigma}_{\varepsilon_{i}}^{2} \lambda_{j}+\mu_{j}\left(1-\rho^{2}+\widehat{\sigma}_{\varepsilon_{i}}^{2}\right) \lambda_{i}}, \text { and } \\
c_{i}^{o} & =\frac{\mu_{j}(1-\rho)\left(1+\rho+\widehat{\sigma}_{\varepsilon_{i}}^{2}\right)}{\mu_{i} \rho \widehat{\sigma}_{\varepsilon_{i}}^{2} \lambda_{j}+\mu_{j}\left(1-\rho^{2}+\widehat{\sigma}_{\varepsilon_{i}}^{2}\right) \lambda_{i}}, \text { where } i, j=1,2, j \neq i .
\end{aligned}
$$

Our next corollary provides some comparative statics results.

Corollary 4. Let $\rho \sigma_{\varepsilon_{1}}^{2} \sigma_{\varepsilon_{2}}^{2}>0$. Then the only equilibrium coefficients affected by $Q, \bar{\theta}_{i}$, and $\bar{\theta}_{j}$ are the intercepts of the demand functions (with $b_{i}^{o}$ increasing in $\bar{\theta}_{i}$ and $Q$ and decreasing in $\bar{\theta}_{j}$ ) for $i, j=1,2$ and $i \neq j$. Furthermore, the demands of group $i$ are less sensitive to private signals and prices (lower $a_{i}$ and $c_{i}$ ) in response to an increase in $\lambda_{i}, \lambda_{j}, \rho, \sigma_{\varepsilon_{i}}^{2}$, and $\mu_{i}$, and to a decrease in $\mu_{j}$; however, group i's demands are not affected by $\sigma_{\varepsilon_{j}}^{2}$.

Observe that, under competitive behavior, we can derive an additional comparative statics result: the relationship between the equilibrium coefficients and the proportion of individuals in group 1. In particular, increasing the proportion $\mu_{1}$ of type-1 traders leads, for those traders, to an increased information-sensitivity weight of the price (higher $\Psi_{1}\left(n_{1} c_{1}^{o}+n_{2} c_{2}^{o}\right)\left(n_{2} a_{2}^{o}\right)^{-1}$ ) and so a lower overall response to the price $\left(c_{1}^{o}=\lambda_{1}^{-1}\left(1-\Psi_{1}\left(n_{1} c_{1}^{o}+n_{2} c_{2}^{o}\right)\left(n_{2} a_{2}^{o}\right)^{-1}\right)\right)$; the opposite holds for type-2 traders.

Thus, as outlined at the beginning of this subsection, the auction outcome can be obtained as the solution to a maximization problem with a more concave objective function than the expected total surplus, which suggests that inefficiency may be eliminated by quadratic subsidies $\left(\kappa_{i} x_{i}^{2} / 2, i=1,2\right)$ that compensate for the distortions. The per capita subsidy rate $\left(\kappa_{i}\right)$ to a trader of type $i$ must be such that it compensates for the distortion $d_{i}\left(\kappa_{i}\right)$ while accounting for the subsidy. Since the aim is to induce competitive behavior, the trader should be led to respond with $c_{i}^{o}$ to the price. This means that the exact amount of $\kappa_{i}$ must be $d_{i}\left(c_{1}^{o}, c_{2}^{o}\right)$, since that would be the distortion arising when traders use the competitive linear strategies. The following proposition shows that, if subsidies are selected properly, then bidders behave competitively and so the equilibrium allocation is efficient.

Proposition 6. Let $i=1,2$ and $i \neq j$. Then the efficient allocation is induced by the quadratic subsidies $\kappa_{i} x_{i}^{2} / 2$, where $\kappa_{i}=d_{i}\left(c_{i}^{o}, c_{j}^{o}\right)=1 /\left(\left(n_{i}-1\right) c_{i}^{o}+n_{j} c_{j}^{o}\right)$. If $\rho \sigma_{\varepsilon_{i}}^{2} \sigma_{\varepsilon_{j}}^{2}>0$, then 
the per capita subsidy rates $\left(\kappa_{i}, i=1,2\right)$ increase with $\rho, \widehat{\sigma}_{\varepsilon_{1}}^{2}, \widehat{\sigma}_{\varepsilon_{2}}^{2}, \lambda_{1}$, and $\lambda_{2}$, but decrease with $n_{1}$ and $n_{2}$. We have that $\kappa_{1}<\kappa_{2}$ iff $c_{1}^{o}<c_{2}^{o}$.

Combining Propositions 5 and 6 now yields closed-form expressions for the optimal subsidy rates:

$$
\kappa_{i}=\frac{1}{n_{j}(1-\rho)}\left(\frac{\left(n_{i}-1\right)\left(1+\widehat{\sigma}_{\varepsilon_{i}}^{2}+\rho\right)}{n_{i} \lambda_{j} \rho \widehat{\sigma}_{\varepsilon_{i}}^{2}+n_{j} \lambda_{i}\left(1-\rho^{2}+\widehat{\sigma}_{\varepsilon_{i}}^{2}\right)}+\frac{n_{i}\left(1+\widehat{\sigma}_{\varepsilon_{j}}^{2}+\rho\right)}{n_{i} \lambda_{j}\left(1-\rho^{2}+\widehat{\sigma}_{\varepsilon_{j}}^{2}\right)+n_{j} \lambda_{i} \rho \widehat{\sigma}_{\varepsilon_{j}}^{2}}\right)^{-1},
$$

$i=1,2, i \neq j$. If $\rho=0$ (or, with full information, if $\sigma_{\varepsilon_{i}}^{2}=0, i=1,2$ ), then $\kappa_{i}^{f}=$ $1 /\left(\left(n_{i}-1\right) \lambda_{i}^{-1}+n_{j} \lambda_{j}^{-1}\right), i=1,2$. Proposition 6 implies that the optimal subsidy rates with incomplete information and learning from prices are higher than with full information: $\kappa_{i}>\kappa_{i}^{f}$ if (a) $\rho>0$ and (b) at least one of $\widehat{\sigma}_{\varepsilon_{1}}^{2}$ or $\widehat{\sigma}_{\varepsilon_{2}}^{2}$ is strictly positive. If $\sigma_{\varepsilon_{1}}^{2}=\infty$, then $\kappa_{i}, i=1,2$, is independent of $\sigma_{\varepsilon_{2}}^{2}$.

Remark 7. With symmetric groups we obtain symmetric optimal subsidies which change as described in Proposition 6 with changes in $\lambda_{i}=\lambda_{j}, \sigma_{\varepsilon_{i}}^{2}=\sigma_{\varepsilon_{j}}^{2}$, and $n_{i}=n_{j}$.

Note that the optimal subsidy rates are decreasing in the number of traders because, when there are many agents, competitive behavior is already being approached in the market without subsidies. Moreover, $\operatorname{sgn}\left\{\kappa_{1}-\kappa_{2}\right\}=\operatorname{sgn}\left\{c_{1}^{o}-c_{2}^{o}\right\}$. Hence $\kappa_{1}<\kappa_{2}$ iff $c_{1}^{o}<c_{2}^{o}$. The implication is that the bidders who require a higher per capita subsidy rate are the ones whose demands are more sensitive to price. Corollary 3 allows us to conclude that, if there is a group with more precise private information, with lower transaction costs, and that is less numerous, then it is the group meriting a higher per capita subsidy rate. The reason is that the stronger group's strategic behavior is more pronounced and so it must receive more compensation in order to become competitive.

The expected (total) optimal subsidy for group $i$ is $\kappa_{i} \mathbb{E}\left[\left(x_{i}^{o}(t)\right)^{2}\right] / 2 .^{29}$ If $\bar{\theta}_{2} \geq \bar{\theta}_{1}$, then $\mathbb{E}\left[\left(x_{2}^{o}(t)\right)^{2}\right]>\mathbb{E}\left[\left(x_{1}^{o}(t)\right)^{2}\right]$, from which it follows that the bidders from the stronger group (group 2) should receive the higher expected subsidy. However, if $\bar{\theta}_{1}>\bar{\theta}_{2}$, then there are parameter configurations under which bidders from the weaker group (group 1) should receive the higher expected subsidy, even though $\kappa_{1}<\kappa_{2}$. These conclusions would have to be revised if redistributive considerations come into play. ${ }^{30}$

Our result has policy implications. It implies, for example, that a central bank seeking an efficient distribution of liquidity among banks should relax collateral requirements (i.e.,

\footnotetext{
${ }^{29}$ We have that $x_{i}^{o}(t)=\left(n_{j}\left(t_{i}-t_{j}\right)+\lambda_{j} Q\right) /\left(n_{i} \lambda_{j}+n_{j} \lambda_{i}\right)$.

${ }^{30}$ Athey et al. (2013) find with regard to US Forest Service timber auctions that restricting entry increases small business participation but substantially reduces efficiency and revenue. In contrast, subsidizing small bidders directly increases revenue and the profits of small bidders without much cost in efficiency. See also Loertscher and Marx (2016) and Pai and Vohra (2012).
} 
provide a larger subsidy) to the strong group. This prescription sounds counterintuitive because the efficiency motive may conflict with the central bank's function as lender of last resort, which often involves shoring up weak banks (e.g., the European Central Bank relaxing the collateral requirements for Greek banks to avoid a meltdown of that country's banking system). Another example is that of a wholesale electricity market characterized by a small (oligopolistic) group and a fringe; in this case, a regulator looking to improve productive efficiency should set a higher subsidy rate for the oligopolistic group. This could be accomplished by offering differential subsidies to renewable energy technologies, for instance, that lower the marginal cost of production.

It is worth noting that primary dealers in the US Treasury are required to bid at least the pro-rate share of those dealers present in the auction ("demonstrate substantial presence") and in exchange enjoy privileges such as exclusive intermediation of OMO, and in the crisis period access to the QE auction mechanism as well as to the Primary Dealer Credit Facility. This may be interpreted as a subsidy that lowers the effective transaction cost of the dealers since they have the obligation to bid a minimum amount.

\subsection{Deadweight loss}

The expected deadweight loss, $E[D W L]$, at an anonymous allocation $\left(x_{1}(t), x_{2}(t)\right)$ is the difference between expected total surplus at the efficient allocation, ET $S^{\circ}$, and at the baseline allocation, denoted simply by ETS. Lemma A4 in Appendix A shows that

$$
\mathbb{E}[D W L]=\frac{1}{2} \lambda_{1} n_{1} \mathbb{E}\left[\left(x_{1}(t)-x_{1}^{o}(t)\right)^{2}\right]+\frac{1}{2} \lambda_{2} n_{2} \mathbb{E}\left[\left(x_{2}(t)-x_{2}^{o}(t)\right)^{2}\right],
$$

where $\left(x_{1}^{o}(t), x_{2}^{o}(t)\right)$ corresponds to the price-taking equilibrium. We can use the equilibrium expressions for $\left(x_{1}(t), x_{2}(t)\right)$ and $\left(x_{1}^{o}(t), x_{2}^{o}(t)\right)$ to show that

$$
\begin{aligned}
\mathbb{E}[D W L]= & \phi\left(\left(n_{2} d_{1}+n_{1} d_{2}\right)^{2} \mathbb{E}\left[\left(t_{1}-t_{2}\right)^{2}\right]\right. \\
& \left.+2\left(n_{2} d_{1}+n_{1} d_{2}\right)\left(\lambda_{2} d_{1}-\lambda_{1} d_{2}\right)\left(\bar{\theta}_{1}-\bar{\theta}_{2}\right) Q+\left(\lambda_{1} d_{2}-\lambda_{2} d_{1}\right)^{2} Q^{2}\right)
\end{aligned}
$$

where $\phi=n_{1} n_{2} /\left(2\left(n_{2} \lambda_{1}+n_{1} \lambda_{2}\right) u^{2}\right)$ and $u=n_{i}\left(d_{j}+\lambda_{j}\right)+n_{j}\left(d_{i}+\lambda_{i}\right)$.

In a double auction $(Q=0)$ or when $\lambda_{2} d_{1}=\lambda_{1} d_{2}$, equation (4) indicates that the noninformational trading terms corresponding to the equilibrium with imperfect competition and the ones corresponding to the competitive equilibrium are both null and, therefore, the expected deadweight loss only derives from differences in the valuation trading terms. Strategic behavior restricts trade and generates inefficiency. ${ }^{31}$

\footnotetext{
${ }^{31}$ Note that if we interpret the traders as producers competing to supply a fixed demand $Q$, then the condition
} 
Consider now the other extreme case where the differences between the equilibrium and efficient quantities arise only in the clearing trading terms (with $\bar{\theta}_{1}=\bar{\theta}_{2}$ and $\rho \rightarrow 1$ ). If group 1 has higher transaction costs $\left(\lambda_{1}>\lambda_{2}\right)$, is more numerous $\left(n_{1}>n_{2}\right)$, and is less informed $\left(\sigma_{\varepsilon_{1}}^{2}>\sigma_{\varepsilon_{2}}^{2}\right)$ than group 2, then $d_{1} / d_{2}<\lambda_{1} / \lambda_{2}$ (and, therefore, $\lambda_{1} d_{2}-\lambda_{2} d_{1}>0$ ). In this case, the weaker group (1) gets more than the efficient allocation $x_{1}(t)>x_{1}^{O}(t)$ (and the stronger one (2) less, $\left.x_{2}(t)<x_{2}^{O}(t)\right)$. Suppose that the weaker group is also the one that ex-ante values less the asset, then $\left(\lambda_{1} d_{2}-\lambda_{2} d_{1}\right)\left(\bar{\theta}_{2}-\bar{\theta}_{1}\right)>0$. Then the differences between the valuation trading terms and the clearing trading terms go in the same direction and inefficiency increases (the second term in the expression of $E[D W L]$ is positive). This is the case with primary dealers in a Treasury auction (strong group) who may value more the bonds than other direct bidders because they have more clout in reselling them. In Hortaçsu et al. (2016) it is found that the willingness to pay of primary dealers is no lower than those of other direct bidders (as well as of indirect bidders). However, group strength and preference strength need not be aligned always. This is the case for example when a weaker group of banks is hit by a crisis, then its valuation for liquidity may increase more than for a strong group of banks.

We can derive the following results.

\section{Proposition 7.}

(i) The expected deadweight loss may be increasing or decreasing in the information parameters $\left(\rho, \widehat{\sigma}_{\varepsilon_{1}}^{2}\right.$, and $\left.\widehat{\sigma}_{\varepsilon_{2}}^{2}\right)$ and, therefore, price impact $\left(d_{1}, d_{2}\right)$ and the $\mathbb{E}[D W L]$ may be negatively associated.

(ii) The $\mathbb{E}[D W L]$ increases with payoff asymmetry and with $Q$ whenever group strength and preference strength are aligned (i.e., when the stronger group 2 , with $\lambda_{1}>\lambda_{2}, n_{1}>n_{2}$, and $\sigma_{\varepsilon_{1}}^{2}>\sigma_{\varepsilon_{2}}^{2}$, values the asset no less than does the weaker group, $\left.\bar{\theta}_{1} \leq \bar{\theta}_{2}\right)$.

(iii) When groups are symmetric, the expected deadweight loss is independent of $Q$, and price impact $d$ and the $\mathbb{E}[D W L \mid t]$ are positively associated, given predicted values $t$, for changes in information parameters. This need not be the case with asymmetric groups (e.g., for $Q$ large, $d_{i} / d_{j}>\lambda_{i} / \lambda_{j}$ implies that $\mathbb{E}[D W L \mid t]$ increases in $d_{i}$ and decreases in $\left.d_{j}\right)$.

Several comments are in order. First, when $(Q=0)$ or when $\lambda_{2} d_{1}=\lambda_{1} d_{2}$,

$$
\mathbb{E}[D W L]=\phi\left(n_{2} d_{1}+n_{1} d_{2}\right)^{2} \mathbb{E}\left[\left(t_{1}-t_{2}\right)^{2}\right]
$$

So, the $\mathbb{E}[D W L]$ is equal to the product of two factors, $\phi\left(n_{2} d_{1}+n_{1} d_{2}\right)^{2}$ which increases in $d_{1}$

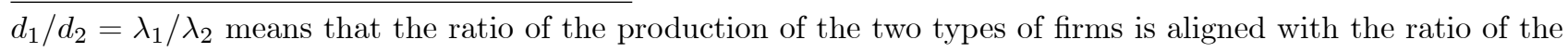
slopes of their respective marginal costs. This condition guarantees productive efficiency provided that $\bar{\theta}_{1}=\bar{\theta}_{2}$ and $\rho=1$ and, since demand is fixed, this coincides with overall efficiency. 
and $d_{2}$, and

$$
\mathbb{E}\left[\left(t_{1}-t_{2}\right)^{2}\right]=\left(\bar{\theta}_{1}-\bar{\theta}_{2}\right)^{2}+(1-\rho)^{2} \sigma_{\theta}^{2} \frac{2(1+\rho)+\widehat{\sigma}_{\varepsilon_{1}}^{2}+\widehat{\sigma}_{\varepsilon_{2}}^{2}}{\left(1+\widehat{\sigma}_{\varepsilon_{1}}^{2}\right)\left(1+\widehat{\sigma}_{\varepsilon_{2}}^{2}\right)-\rho^{2}},
$$

which decreases in $\rho$ and in $\widehat{\sigma}_{\varepsilon_{i}}^{2}$, vanishes when $\rho$ approaches 1 or when there is no uncertainty $\left(\sigma_{\theta}^{2}=0\right)$ provided $\bar{\theta}_{1}=\bar{\theta}_{2}$, and increases in $\left(\bar{\theta}_{1}-\bar{\theta}_{2}\right)^{2}$. It is worth noting that both $\operatorname{ETS}^{o}$ and ETS increase in $E\left[\left(t_{1}-t_{2}\right)^{2}\right]$, which is associated to gains from trade by the two groups. However, the weight of $E\left[\left(t_{1}-t_{2}\right)^{2}\right]$ in $E T S^{\circ}$ is larger than in ETS and, therefore, E[DWL] increases in $E\left[\left(t_{1}-t_{2}\right)^{2}\right]$.

Since $d_{1}$ and $d_{2}$ increase in $\rho$ and $\sigma_{\varepsilon_{i}}^{2}$, we have that $\phi\left(n_{2} d_{1}+n_{1} d_{2}\right)^{2}$ increases in $\rho$ and $\sigma_{\varepsilon_{i}}^{2}$. It follows that $E[D W L]$ may increase or decrease in $\rho$ and $\widehat{\sigma}_{\varepsilon_{i}}^{2}$, as indicated in Proposition 7 (i). However, if $\sigma_{\varepsilon_{i}}^{2}=\infty$, then $d_{i}$ is independent of $\sigma_{\varepsilon_{j}}^{2}, i=1,2, j \neq i$, and $E[D W L]$ decreases with $\sigma_{\varepsilon_{j}}^{2}$. Under full information (i.e., $\sigma_{\varepsilon_{1}}^{2}=\sigma_{\varepsilon_{2}}^{2}=0$ ), both $d_{1}$ and $d_{2}$ are independent of $\rho$; in this case $E[D W L]$ decreases with $\rho$. Similarly, if $\rho=0$, then $d_{1}$ and $d_{2}$ are independent of $\sigma_{\varepsilon_{1}}^{2}$ and $\sigma_{\varepsilon_{2}}^{2}$, from which it follows that $E[D W L]$ decreases with $\sigma_{\varepsilon_{1}}^{2}$ and $\sigma_{\varepsilon_{2}}^{2}$.

Second, the impact of a small amount of asymmetry may be large. Suppose, for example, that the initial situation is symmetric for the groups and that $\sigma_{\theta}^{2}$ is low. Then, the $E[D W L]$ is close to zero since we have that $d_{1} / d_{2}=\lambda_{1} / \lambda_{2}$. However, if $\lambda_{2}$ is lowered somewhat, then we know from Proposition 3 (iii) that $d_{1} / d_{2}$ decreases and, therefore, $d_{1} / d_{2}<\lambda_{1} / \lambda_{2}$, in which case $E[D W L]$ may be quite large if $Q$ is large. This is consistent with the results in Hortaçsu et al. (2016) who document a significant amount of efficiency losses due to heterogeneity at long maturities in US Treasury auctions.

\subsection{Market integration}

Our analysis can shed light also on the effects of integrating separated markets. ${ }^{32}$ Suppose that groups 1 and 2 operate in separate markets (auctions), that is, in market $i$ all the buyers $\left(n_{i}\right)$ are of type $i$ and supply is $n_{i} Q /\left(n_{1}+n_{2}\right)$. In this framework, given that all the individuals are identical in market $i$, the market clearing condition implies that the equilibrium quantities are given by $Q /\left(n_{1}+n_{2}\right)$. Let $(1-\rho) \sigma_{\theta}^{2}>0$, then market integration (a unified auction):

1. Increases ETS if bidders behave competitively (strictly except if $\bar{\theta}_{1}=\bar{\theta}_{2}, \sigma_{\varepsilon_{1}}^{2}=\sigma_{\varepsilon_{2}}^{2}=\infty$, and $\lambda_{1}=\lambda_{2}$ ). In the latter case payoffs are symmetric among bidders of the two groups and there is no information on values. Therefore, there are no gains from trade among the groups.

\footnotetext{
${ }^{32}$ See Appendix B for a more developed analysis and proof of the results.
} 
2. Increases ETS, because of gains from trade, provided $\sigma_{\varepsilon_{i}}^{2}<\infty$ for some group $i$, if $\bar{\theta}_{1} \neq \bar{\theta}_{2}$ and $Q=0$, or if groups are symmetric.

3. May decrease $E T S$, if $Q$ is large and the groups asymmetric. In this case gains from trade of integration are overpowered by the inefficiency generated by group asymmetries and price impact. This may happen with no asymmetric information but asymmetric payoffs. For example, if $\bar{\theta}_{1}=\bar{\theta}_{2}, \sigma_{\varepsilon_{1}}^{2}=\sigma_{\varepsilon_{2}}^{2}=\infty, \lambda_{1}>\lambda_{2}$, and $d_{1}+\lambda_{1}<d_{2}+\lambda_{2}$. In this case group 2 (with lower transaction costs) obtains a lower quantity in the integrated market because of higher price impact (see Expression (4)) and diminishes total surplus. Note that this could not happen if traders behaved competitively. With asymmetric precisions of information $\left(\sigma_{\varepsilon_{1}}^{2} \neq \sigma_{\varepsilon_{2}}^{2}\right)$ and incomplete information $(\rho>0)$ but otherwise symmetric groups, integration may be also welfare decreasing for large $Q$. Note that this would not happen with complete information, $\rho=0$.

\section{$5 \quad$ Large markets}

Our objective in this section is to determine whether (or not) the equilibrium under imperfect competition converges to a price-taking equilibrium in the limit as the number of traders becomes large. We examine two possible scenarios: in the first, only group 1 is large; in the second, both groups of bidders are large. The per capita supply (denoted by $q$ ) is assumed to be inelastic, that is, $Q=\left(n_{1}+n_{2}\right) q$.

\subsection{Oligopsony with competitive fringe}

Proposition 8. Let $\rho \sigma_{\varepsilon_{1}}^{2}>0$. Suppose that $n_{1} \rightarrow \infty$ and $n_{2}<\infty$. Then an equilibrium exists iff $n_{2}>\bar{n}_{2}\left(\rho, \widehat{\sigma}_{\varepsilon_{1}}^{2}, \widehat{\sigma}_{\varepsilon_{2}}^{2}\right)$, where $\bar{n}_{2}$ is increasing in $\rho$ and $\widehat{\sigma}_{\varepsilon_{1}}^{2}$ and where $\bar{n}_{2}$ is decreasing in $\widehat{\sigma}_{\varepsilon_{2}}^{2}$ whenever $(2 \rho-1) \widehat{\sigma}_{\varepsilon_{1}}^{2}<1-\rho^{2}$. An agent in the large group absorbs the inelastic per capita supply in the limit $\left(\lim _{n_{1} \rightarrow \infty} b_{1}=q, \lim _{n_{1} \rightarrow \infty} a_{1}=\lim _{n_{1} \rightarrow \infty} c_{1}=0\right)$ and retains some price impact $\left(\lim _{n_{1} \rightarrow \infty} d_{1}>\right.$ $0)$, while an agent in the small group commands a higher degree of market power $\left(\lim _{n_{1} \rightarrow \infty} d_{2}>\right.$ $\left.\lim _{n_{1} \rightarrow \infty} d_{1}\right)$.

When $n_{2}=1$, the existence condition stated in Proposition 8 boils down to $(2 \rho-1) \widehat{\sigma}_{\varepsilon_{1}}^{2}<$ $1-\rho^{2}$ from Corollary 1. Equation (48) in Appendix A shows that, when $n_{2}=\bar{n}_{2}\left(\rho, \widehat{\sigma}_{\varepsilon_{1}}^{2}, \widehat{\sigma}_{\varepsilon_{2}}^{2}\right)$, the demand functions for bidders in group 2 would be completely inelastic $\left(\lim _{n_{1} \rightarrow \infty} c_{2}=0\right)$. This explains why the inequality $n_{2}>\bar{n}_{2}\left(\rho, \widehat{\sigma}_{\varepsilon_{1}}^{2}, \widehat{\sigma}_{\varepsilon_{2}}^{2}\right)$ is required for the existence of equilibrium. Neither group 1 nor group 2 has flat aggregate demand in the limit, and each group has some 
price impact. We see that an agent in the large group just absorbs the inelastic per capita supply, behaving like a "Cournot quantity setter", and keeping some price impact $\left(\lim _{n_{1} \rightarrow \infty} d_{1}>0\right)$, while bidders in the small group command relatively more market power $\left(\lim _{n_{1} \rightarrow \infty} d_{2}>\lim _{n_{1} \rightarrow \infty} d_{1}\right)$. It is worth to remark that the large group retains price impact in the limit only if this group learns from the price (incomplete information and correlation of values, $\rho \sigma_{\varepsilon_{1}}^{2}>0$ ). In this case the aggregate demand of group 1 does not become flat, $\lim _{n_{1} \rightarrow \infty} n_{1} c_{1}<\infty$. If $\rho \sigma_{\varepsilon_{1}}^{2}=0, \lim _{n_{1} \rightarrow \infty} n_{1} c_{1}=\infty$ and there is no price impact in the limit: $\lim _{n_{1} \rightarrow \infty} d_{i}=0, i=1,2$. It is easy to see also that, in the limit, the price depends only on the valuations and price impact of agents in the competitive fringe: $\lim _{n_{1} \rightarrow \infty} p=\mathbb{E}\left[\theta_{1} \mid s_{1}, s_{2}\right]-\left(\lim _{n_{1} \rightarrow \infty} d_{1}+\lambda_{1}\right) q \cdot{ }^{33}$

If the small group is fully informed $\left(\sigma_{\varepsilon_{2}}^{2}=0\right)$ and the large group is entirely uninformed $\left(\sigma_{\varepsilon_{1}}^{2} \rightarrow \infty\right)$, then: $\bar{n}_{2}=2 \rho$; an equilibrium always exists for $n_{2}>2$; and the equilibrium coefficients for group 2 are $\lim _{n_{1} \rightarrow \infty} b_{2}=0$, and $\lim _{n_{1} \rightarrow \infty} a_{2}=\lim _{n_{1} \rightarrow \infty} c_{2}=\left(n_{2}-2 \rho\right) /\left(\left(n_{2}-\rho\right) \lambda_{2}\right)$. In this case, the groups' relative price impact is given by $\lim _{n_{1} \rightarrow \infty}\left(d_{2} / d_{1}\right)=1+\rho /\left(n_{2}-\rho\right)$. As $\rho$ increases the relative price impact of group 2 increases also. This accords with the general theme that increased correlation in the presence of asymmetric information raises price impact.

\subsection{A large price-taking market}

Consider now the following setup. There is a continuum of bidders along the interval $[0,1]$, and we let $q$ denote the aggregate (average) quantity supplied in the market. Suppose that a fraction $\mu_{i}\left(0<\mu_{i}<1\right)$ of these bidders are traders of type $i, i=1,2$. Then the following proposition characterizes the equilibrium of this continuum economy and shows that it is the limit of a finite economy's equilibrium.

Proposition 9. Let $Q=\left(n_{1}+n_{2}\right) q$. Suppose that $n_{1}$ and $n_{2}$ both approach to infinity and that $n_{i} /\left(n_{1}+n_{2}\right)$ converges to $\mu_{i}\left(0<\mu_{i}<1\right)$ for $i=1,2$. Then, the equilibrium coefficients converge to the equilibrium coefficients of the equilibrium of the continuum economy setup, which coincide with the equilibrium coefficients of the competitive setup (whose expressions are given in the statement of Proposition 5).

How do prices compare under imperfect competition and under perfect competition? It is easy to see that the expected competitive price $\mathbb{E}\left(p^{o}\right)$ is given by Expression (6) letting

\footnotetext{
${ }^{33}$ The limit expected quantity of a bidder of group 2 is given by $\lim _{n_{1} \rightarrow \infty} \mathbb{E}\left[x_{2}(t)\right]=$ $\left(\bar{\theta}_{2}-\bar{\theta}_{1}+\left(\lim _{n_{1} \rightarrow \infty} d_{1}+\lambda_{1}\right) q\right) /\left(\lim _{n_{1} \rightarrow \infty} d_{2}+\lambda_{2}\right)$.
} 
$d_{2}=d_{1}=0$. It follows that

$\operatorname{sgn}\left\{\mathbb{E}(p)-\mathbb{E}\left(p^{o}\right)\right\}=\operatorname{sgn}\left\{\left(\lambda_{1} d_{2}-\lambda_{2} d_{1}\right)\left(\bar{\theta}_{1}-\bar{\theta}_{2}\right)-\left(n_{2}^{-1} \lambda_{2} d_{1}\left(d_{2}+\lambda_{2}\right)+n_{1}^{-1} \lambda_{1} d_{2}\left(d_{1}+\lambda_{1}\right)\right) Q\right\}$

Notice that when $\lambda_{1} d_{2}=\lambda_{2} d_{1}$ (as in the symmetric case) or when $\bar{\theta}_{1}=\bar{\theta}_{2}, E(p)<E\left(p^{o}\right)$ whenever $Q>0$. We have then the expected result that market power lowers prices (recall that we are in bidding/demand competition). However, whenever $Q$ is low enough and $\left(\lambda_{1} d_{2}-\lambda_{2} d_{1}\right)\left(\bar{\theta}_{1}-\bar{\theta}_{2}\right)>0$, we have that $\mathbb{E}(p)>\mathbb{E}\left(p^{o}\right)$. This is so, in particular, if group 1 is weak $\left(\lambda_{1}>\lambda_{2}, \sigma_{\varepsilon_{1}}^{2}>\sigma_{\varepsilon_{2}}^{2}, n_{1}>n_{2}\right)$, which implies $d_{1} / d_{2}<\lambda_{1} / \lambda_{2}$, and values the asset more $\left(\bar{\theta}_{1}>\bar{\theta}_{2}\right)$. When this happens the price intercept of expected aggregate inverse demand is lower in the competitive case and since the slope is also lower than with imperfect competition we have that the expected competitive price is lower than the expected oligopoly price when $Q$ is low (see Figure 2). Precisely when $\left(\lambda_{1} d_{2}-\lambda_{2} d_{1}\right)\left(\bar{\theta}_{1}-\bar{\theta}_{2}\right)=0$ the price intercept of the competitive and oligopoly aggregate demands coincide.

[Figure 2 here]

\section{Supply function competition}

The case of supply schedule competition for inelastic demand is easily accommodated by considering negative demands $\left(x_{i}<0\right)$ and a negative inelastic supply $(Q<0)$. In this case, a producer of type $i$ has a quadratic production cost $-\theta_{i} x_{i}+\lambda_{i} x_{i}^{2} / 2$. This case is empirically relevant in markets such as wholesale electricity, where concentration is high also. ${ }^{34} \mathrm{~A}$ number of empirical studies have concluded that sellers have exercised significant market power in wholesale electricity markets (see, e.g., Green and Newbery 1992; Wolfram 1998; Borenstein et al. 2002; Joskow and Kahn 2002). Most wholesale electricity markets prefer using a uniformprice auction to using a pay-as-bid auction (Cramton and Stoft 2006, 2007). In several of these markets (e.g., California, Australia), generating companies bid to sell power and wholesale customers bid to buy power. In such markets, asymmetries are prevalent. For example, some generators of wholesale electricity rely heavily on nuclear technology, which has flat marginal costs, whereas others rely mostly on fuel technologies, which have steep marginal costs. Holmberg and Wolak (2015) argue that, in wholesale electricity markets, information on suppliers'

\footnotetext{
${ }^{34}$ European Commission (2007) has asserted that "at the wholesale level, gas and electricity markets remain national in scope, and generally maintain the high level of concentration of the pre-liberalization period. This gives scope for exercising market power" (Inquiry pursuant to Article 17 of Regulation (EC) No 1/2003 into the European gas and electricity sectors (Final Report), Brussels, 10.1.2007).
} 
production costs is asymmetric. For evidence on the effect of cost heterogeneity on bidding in wholesale electricity markets, see Crawford et al. (2007) and Bustos-Salvagno (2015). ${ }^{35}$

The result that if a group of traders is stronger in the sense described previously (i.e., if its private information is more precise, its transaction costs are lower, and it is more oligopolistic), then the members of that group react more (than do the bidders of the other group) to the private signal and also to the price, may help explain the finding of Hortaçsu and Puller (2008) for the Texas balancing market where, with no accounting for private information on costs, small firms use steeper schedules than the theory would predict.

\section{Concluding remarks}

We analyze a divisible good uniform-price auction, where two types of bidders compete. Each of these two groups contains a finite number of identical bidders. At the unique equilibrium, a group's relative price impact increases with the precision of private information and decreases with the group's transaction costs. Consistently with the empirical evidence for liquidity auctions (Cassola et al. 2013), we find that an increase in the transaction costs of a group of bidders induces a strategic response from the other group, whose members then submit steeper schedules. The effect is reinforced with the impact of changes in the information parameters in a crisis situation. The comparative static results obtained provide testable predictions. For example, an increase in transaction cost or noise in the signals in any group, or an increase in correlation of values across groups, should increase the price impact of traders in both groups. Furthermore, co-movements in those parameters magnify the impact. The group that is stronger (because it has more precise private information, faces lower transaction costs, and is more oligopsonistic) has more price impact and must therefore receive a higher subsidy to behave competitively. This result is consistent with the evidence of US Treasury auctions (Hortaçsu et al. 2016) where primary dealers (strong group) exercise market power and earn significant surplus on top of having privileges in exchange for bidding minimum amounts in the auctions. The expected deadweight loss increases with the quantity auctioned and with the degree of payoff asymmetry provided the stronger group values the asset no less than does the weaker group. A small amount of asymmetry may generate large deadweight losses. The link of heterogeneity and

\footnotetext{
${ }^{35}$ Linear supply function models have been used extensively for estimating market power in wholesale electricity auctions. Holmberg et al. (2013) provide a foundation for the continuous approach as an approximation to the discrete supply bids in a spot market. In their experimental work, Brandts et al. (2014) find that observed behavior is more consistent with a supply function model than with a discrete multi-unit auction model. Ciarreta and Espinosa (2010) use Spanish data in finding more empirical support for the smooth supply model than the discrete-bid auction model.
} 
efficiency losses is corroborated empirically for Treasury auctions by Hortaçsu et al. (2016).

Our findings have policy implications. Consider a regulator who wants to reduce inefficiency in an industry with two groups of firms (e.g., a small oligopolistic group and a competitive fringe). This regulator must bear in mind that any intervention directed toward one group will also affect the other's behavior. In addition, the regulator should set a higher subsidy rate for the group that has better information, is more oligopsonistic, and has lower transaction costs. The framework developed here can be adapted to study competition policy analyzing the effects of merger and industry capacity redistribution.

\section{Appendix A}

Proposition 1 follows from Lemmata A1 and A2.

Lemma A1. Let $\rho<1$. In equilibrium, the demand function for a trader of type $i, i=1,2$, is given by $X_{i}\left(s_{i}, p\right)=\left(\mathbb{E}\left[\theta_{i} \mid s_{i}, p\right]-p\right) /\left(d_{i}+\lambda_{i}\right)$, with $d_{i}+\lambda_{i}>0$. The equilibrium coefficients satisfy the following system of equations:

$$
\begin{aligned}
b_{i} & =\left(\left(1-\Xi_{i}\right) \bar{\theta}_{i}-\Psi_{i} \bar{\theta}_{j}-\frac{\Psi_{i}\left(n_{i} b_{i}+n_{j} b_{j}-Q\right)}{n_{j} a_{j}}\right) /\left(d_{i}+\lambda_{i}\right), \\
a_{i} & =\left(\Xi_{i}-\frac{n_{i} a_{i}}{n_{j} a_{j}} \Psi_{i}\right) /\left(d_{i}+\lambda_{i}\right), \text { and } \\
c_{i} & =\left(1-\frac{\Psi_{i}\left(n_{i} c_{i}+n_{j} c_{j}\right)}{n_{j} a_{j}}\right) /\left(d_{i}+\lambda_{i}\right),
\end{aligned}
$$

where $i, j=1,2, j \neq i$. Moreover, in equilibrium, $a_{i}>0, i=1,2$.

Proof: Consider a trader of type $i$. Recall that at the beginning of Subsection 3.1 we obtain $X_{i}\left(s_{i}, p\right)=\left(\mathbb{E}\left[\theta_{i} \mid s_{i}, p\right]-p\right) /\left(d_{i}+\lambda_{i}\right)$ and $\mathbb{E}\left[\theta_{i} \mid s_{i}, p\right]=\mathbb{E}\left[\theta_{i} \mid s_{i}, s_{j}\right]$. Since we are looking for

strategies of the form $X_{i}\left(s_{i}, p\right)=b_{i}+a_{i} s_{i}-c_{i} p$, from the market clearing condition we have that $s_{j}=\left(\left(n_{i} c_{i}+n_{j} c_{j}\right) p+Q-n_{i}\left(b_{i}+a_{i} s_{i}\right)-n_{j} b_{j}\right) /\left(n_{j} a_{j}\right)$. Thus, from (3), it follows that

$\mathbb{E}\left[\theta_{i} \mid s_{i}, s_{j}\right]=\left(1-\Xi_{i}\right) \bar{\theta}_{i}-\Psi_{i} \bar{\theta}_{j}+\Psi_{i}\left(\frac{Q-n_{i} b_{i}-n_{j} b_{j}}{n_{j} a_{j}}\right)+\left(\Xi_{i}-\frac{n_{i} a_{i}}{n_{j} a_{j}} \Psi_{i}\right) s_{i}+\Psi_{i}\left(\frac{n_{i} c_{i}+n_{j} c_{j}}{n_{j} a_{j}}\right) p$

Substituting the foregoing expression in (1), and then identifying coefficients, we obtain the expressions for the demand coefficients given in (8)-(10).

Finally, we show the positiveness of the coefficients $a_{i}, i=1,2$. From (9), we get $a_{i}=$ $\Xi_{i} /\left(d_{i}+\lambda_{i}+n_{i} \Psi_{i} /\left(n_{j} a_{j}\right)\right), i, j=1,2, j \neq i$. Combining the previous expressions, we have

$$
a_{i}=\frac{n_{j}\left(\Xi_{i} \Xi_{j}-\Psi_{i} \Psi_{j}\right)}{n_{i} \Psi_{i}\left(d_{j}+\lambda_{j}\right)+\Xi_{j} n_{j}\left(d_{i}+\lambda_{i}\right)}, i, j=1,2, j \neq i .
$$


Direct computation yields $\Xi_{i} \Xi_{j}-\Psi_{i} \Psi_{j}=\left(1-\rho^{2}\right) /\left(\left(1+\widehat{\sigma}_{\varepsilon_{1}}^{2}\right)\left(1+\widehat{\sigma}_{\varepsilon_{2}}^{2}\right)-\rho^{2}\right)>0$, whenever $\rho<1$. Moreover, using the positiveness of $d_{i}+\lambda_{i}, \Xi_{i}$, and $\Psi_{i}, i=1,2$, we conclude that, in equilibrium, the coefficients $a_{i}, i=1,2$, are strictly positive.

Lemma A2. In equilibrium,

$$
\begin{aligned}
b_{i} & =\frac{\Psi_{i}}{n_{i} n_{j}} \frac{n_{i} \Xi_{j} \frac{a_{i}}{a_{j}}-n_{j} \Psi_{j}}{\Xi_{i} \Xi_{j}-\Psi_{i} \Psi_{j}} Q+a_{i}\left(\frac{\Xi_{j} \bar{\theta}_{i}-\Psi_{i} \bar{\theta}_{j}}{\Xi_{i} \Xi_{j}-\Psi_{i} \Psi_{j}}-\bar{\theta}_{i}\right), \\
a_{i} & =\Delta_{i} c_{i}, \\
c_{1} & =\left(\Xi_{1} \Delta_{1}^{-1}-\frac{n_{1}}{n_{2}}\left(1-\Xi_{1} \Delta_{1}^{-1}\right) z-\frac{z}{\left(n_{1}-1\right) z+n_{2}}\right) / \lambda_{1}, \text { and } \\
c_{2} & =\left(\Xi_{2} \Delta_{2}^{-1}-\frac{n_{2}}{n_{1}}\left(1-\Xi_{2} \Delta_{2}^{-1}\right) \frac{1}{z}-\frac{1}{n_{1} z+n_{2}-1}\right) / \lambda_{2},
\end{aligned}
$$

where $\Delta_{i}=1 /\left(1+(1+\rho)^{-1} \widehat{\sigma}_{\varepsilon_{i}}^{2}\right), i, j=1,2, j \neq i$. Moreover, $z \equiv c_{1} / c_{2}$ is the unique positive solution to the cubic polynomial $g(z)=g_{3} z^{3}+g_{2} z^{2}+g_{1} z+g_{0}$, with

$$
\begin{aligned}
g_{3}= & n_{1}^{2}\left(n_{1}-1\right)\left(n_{2} \Xi_{2} \Delta_{2}^{-1} \lambda_{1}+n_{1}\left(1-\Xi_{1} \Delta_{1}^{-1}\right) \lambda_{2}\right), \\
g_{2}= & n_{1}\left(\left(3 n_{2} n_{1}-n_{1}-2 n_{2}+1\right)\left(n_{2} \Xi_{2} \Delta_{2}^{-1} \lambda_{1}-n_{1} \Xi_{1} \Delta_{1}^{-1} \lambda_{2}\right)+\right. \\
& \left.+\lambda_{2} n_{1}\left(2 n_{2} n_{1}-n_{1}+1\right)-\left(n_{1}-1\right)\left(n_{2}+1\right) n_{2} \lambda_{1}\right), \\
g_{1}= & n_{2}\left(\left(3 n_{2} n_{1}-2 n_{1}-n_{2}+1\right)\left(n_{2} \Xi_{2} \Delta_{2}^{-1} \lambda_{1}-n_{1} \Xi_{1} \Delta_{1}^{-1} \lambda_{2}\right)+\right. \\
& \left.+\lambda_{2} n_{1}\left(n_{2}-1\right)\left(n_{1}+1\right)-\left(2 n_{2} n_{1}-n_{2}+1\right) n_{2} \lambda_{1}\right), \text { and } \\
g_{0}= & -n_{2}^{2}\left(n_{2}-1\right)\left(n_{2}\left(1-\Xi_{2} \Delta_{2}^{-1}\right) \lambda_{1}+n_{1} \Xi_{1} \Delta_{1}^{-1} \lambda_{2}\right) .
\end{aligned}
$$

Proof: In relation to the expression for $b_{i}$, notice that (9) implies

$$
d_{i}+\lambda_{i}=\left(\Xi_{i}-\frac{n_{i} a_{i}}{n_{j} a_{j}} \Psi_{i}\right) / a_{i}, i, j=1,2, j \neq i
$$

Substituting these expressions in (8), it follows that

$$
b_{i}=a_{i} \frac{\left(1-\Xi_{i}\right) \bar{\theta}_{i}-\Psi_{i} \bar{\theta}_{j}-\frac{\Psi_{i}\left(n_{i} b_{i}+n_{j} b_{j}-Q\right)}{n_{j} a_{j}}}{\Xi_{i}-\frac{n_{i} a_{i}}{n_{j} a_{j}} \Psi_{i}}, i, j=1,2, j \neq i .
$$

Thus,

$n_{i} b_{i}+n_{j} b_{j}=n_{i} a_{i} \frac{\left(1-\Xi_{i}\right) \bar{\theta}_{i}-\Psi_{i} \bar{\theta}_{j}-\frac{\Psi_{i}\left(n_{i} b_{i}+n_{j} b_{j}-Q\right)}{n_{j} a_{j}}}{\Xi_{i}-\frac{n_{i} a_{i}}{n_{j} a_{j}} \Psi_{i}}+n_{j} a_{j} \frac{\left(1-\Xi_{j}\right) \bar{\theta}_{j}-\Psi_{j} \bar{\theta}_{i}-\frac{\Psi_{j}\left(n_{i} b_{i}+n_{j} b_{j}-Q\right)}{n_{i} a_{i}}}{\Xi_{j}-\frac{n_{j} a_{j}}{n_{i} a_{i}} \Psi_{j}}$,

Isolating $n_{i} b_{i}+n_{j} b_{j}$ in the previous formula and substituting the resulting expression in (17), Expression (12) is obtained. 
Concerning the expression for $a_{i}$, substituting (16) in (10), it follows that

$$
c_{i}=a_{i}\left(1-\frac{\Psi_{i}\left(n_{i} c_{i}+n_{j} c_{j}\right)}{n_{j} a_{j}}\right) /\left(\Xi_{i}-\frac{n_{i} a_{i}}{n_{j} a_{j}} \Psi_{i}\right), i, j=1,2, j \neq i .
$$

Hence, $n_{i} c_{i}+n_{j} c_{j}=n_{i} a_{i} \frac{n_{j} a_{j}-\Psi_{i}\left(n_{i} c_{i}+n_{j} c_{j}\right)}{n_{j} a_{j} \Xi_{i}-n_{i} a_{i} \Psi_{i}}+n_{j} a_{j} \frac{n_{i} a_{i}-\Psi_{j}\left(n_{i} c_{i}+n_{j} c_{j}\right)}{n_{i} a_{i} \Xi_{j}-n_{j} a_{j} \Psi_{j}}$. Isolating $n_{i} c_{i}+n_{j} c_{j}$ in the previous formula and substituting the resulting expression in (18), we obtain a formula which is equivalent to (13). Using (13) in (10), we get the expression for $c_{i}$ given in the statement of Proposition 1.

In relation to $c_{1}$ and $c_{2}$, using the expression for $d_{i}$ and (13), (16) implies that

$$
\lambda_{i}=\left(\frac{\Xi_{i}}{\Delta_{i}}-\frac{n_{i} \Psi_{i} c_{i}}{n_{j} \Delta_{j} c_{j}}\right) c_{i}^{-1}-\left(\left(n_{i}-1\right) c_{i}+n_{j} c_{j}\right)^{-1}, i, j=1,2, j \neq i .
$$

or, since

$$
\Psi_{i} \Delta_{j}^{-1}=1-\Xi_{i} \Delta_{i}^{-1}
$$

$\lambda_{i}=\left(\Xi_{i} \Delta_{i}^{-1}-\frac{n_{i}}{n_{j}}\left(1-\Xi_{i} \Delta_{i}^{-1}\right) \frac{c_{i}}{c_{j}}\right) c_{i}^{-1}-\left(\left(n_{i}-1\right) c_{i}+n_{j} c_{j}\right)^{-1}, i, j=1,2, j \neq i$, which imply (14) and (15) since $z=c_{1} / c_{2}$. Moreover, dividing the previous two equalities, it follows that

$$
\frac{\lambda_{1}}{\lambda_{2}}=\frac{\Xi_{1} \Delta_{1}^{-1}-\frac{n_{1}}{n_{2}}\left(1-\Xi_{1} \Delta_{1}^{-1}\right) z-z\left(\left(n_{1}-1\right) z+n_{2}\right)^{-1}}{\Xi_{2} \Delta_{2}^{-1} z-\frac{n_{2}}{n_{1}}\left(1-\Xi_{2} \Delta_{2}^{-1}\right)-z\left(n_{1} z+n_{2}-1\right)^{-1}} .
$$

After some algebra, (20) is equivalent to $g(z)=0$, where $g(z)=g_{3} z^{3}+g_{2} z^{2}+g_{1} z+g_{0}$, as stated in this lemma. Notice that $g(0)<0$ and $\lim _{z \rightarrow \infty} g(z)=\infty$. Consequently, there exists $z \in(0, \infty)$ such that $g(z)=0$. Furthermore, we have that $g_{2} / n_{1}>g_{1} / n_{2}$. Combining this inequality with the fact that $g_{3}>0$ and $g_{0}<0$ allows us to conclude that at least there is only one sign change of the coefficients of $g(z)$. To show that, we distinguish 3 cases:

(1) $0 \geq \frac{g_{2}}{n_{1}}>\frac{g_{1}}{n_{2}}$. This implies that $0 \geq g_{2}$ and $0>g_{1}$. As $g_{3}>0$ and $g_{0}<0$, we conclude that there is only one sign change of the coefficients of $g(z)$.

(2) $\frac{g_{2}}{n_{1}}>0 \geq \frac{g_{1}}{n_{2}}$. This implies that $g_{2}>0 \geq g_{1}$. As $g_{3}>0$ and $g_{0}<0$, we conclude that there is only one sign change of the coefficients of $g(z)$.

(3) $\frac{g_{2}}{n_{1}}>\frac{g_{1}}{n_{2}}>0$. This implies that $g_{2}>0$ and $g_{1}>0$. As $g_{3}>0$ and $g_{0}<0$, we conclude that there is only one sign change of the coefficients of $g(z)$.

This property implies that there exists only one sign change in the coefficients of $g(z)$. Applying the Descartes' rule, we conclude that there exists a unique positive root of $g(z)$.

Proposition 2A. Let $\rho<1$.

(a) There exists an equilibrium if and only if $c_{i}>0, i=1,2$, where

$$
c_{1}=\frac{q_{N}(z)}{\left(\left(n_{1}-1\right) z+n_{2}\right) n_{2} \lambda_{1}} \text { and } c_{2}=\frac{q_{D}(z)}{\left(n_{1} z+n_{2}-1\right) n_{1} z \lambda_{2}},
$$


where $z=c_{1} / c_{2}$ and the expressions of $q_{N}(Z)$ and $q_{D}(Z)$ are given by

$$
\begin{aligned}
& q_{N}(Z)=n_{2}^{2} \Xi_{1} \Delta_{1}^{-1}+n_{2}\left(\Xi_{1} \Delta_{1}^{-1}\left(2 n_{1}-1\right)-\left(n_{1}+1\right)\right) Z-\left(n_{1}-1\right)\left(1-\Xi_{1} \Delta_{1}^{-1}\right) n_{1} Z^{2} \text { and } \\
& q_{D}(Z)=-n_{2}\left(n_{2}-1\right)\left(1-\Xi_{2} \Delta_{2}^{-1}\right)+n_{1}\left(\Xi_{2} \Delta_{2}^{-1}\left(2 n_{2}-1\right)-\left(n_{2}+1\right)\right) Z+n_{1}^{2} \Xi_{2} \Delta_{2}^{-1} Z^{2} .
\end{aligned}
$$

(b) Complete information. When $\rho=0$, or $\sigma_{\varepsilon_{i}}^{2}=0$, or $\sigma_{\varepsilon_{i}}^{2}=\infty, i=1,2$, equilibrium exists iff $n_{1}+n_{2} \geq 3$.

(c) Incomplete information.

(c.1) Let $\rho \sigma_{\varepsilon_{1}}^{2} \sigma_{\varepsilon_{2}}^{2}>0$. Then $c_{i}>0(i=1,2)$ if and only if $\bar{z}_{N}>\bar{z}_{D}$, where $\bar{z}_{N}$ and $\bar{z}_{D}$ denote the highest root of $q_{N}(Z)$ and $q_{D}(Z)$, respectively.

(c.2) Let $\rho \sigma_{\varepsilon_{i}}^{2}>0$ and $\sigma_{\varepsilon_{j}}^{2}=0, i \neq j$. Then $c_{i}>0(i=1,2)$ if $n_{j} \geq 2$, or if $n_{j}=1, n_{i}$ large enough and $\rho$ low enough.

Remark 8. For an equilibrium to exist we must have $c_{i}>0(i=1,2)$ and these inequalities hold if and only if $\bar{z}_{D}<z<\bar{z}_{N}$. If $n_{1}=1$ and $n_{2}=1$, then $\bar{z}_{N}=1 /\left(2 \Delta_{1} \Xi_{1}^{-1}-1\right)$ and $\bar{z}_{D}=2 \Delta_{2} \Xi_{2}^{-1}-1$. Since $\Delta_{i} \Xi_{i}^{-1} \geq 1, i=1,2$, and $\Delta_{1} \Xi_{1}^{-1}=\Delta_{2} \Xi_{2}^{-1}=1$ do not hold, we can use direct computation to obtain $\bar{z}_{N}<\bar{z}_{D}$. Applying Proposition 2A, we conclude that no equilibrium exists in this case. Therefore, $n_{1}+n_{2} \geq 3$ is a necessary condition for the existence of an equilibrium.

Remark 9. In c.1, we obtain that $\lim _{\lambda_{1} \rightarrow 0} z=\bar{z}_{N}$ and $\lim _{\lambda_{2} \rightarrow 0} z=\bar{z}_{D}$.

Remark 10. In c.2, when $\sigma_{\varepsilon_{2}}^{2}=0, \bar{z}_{D}=1 / n_{1}$ if $n_{2}=1$, whereas $\bar{z}_{D}=0$ if $n_{2} \geq 2$.

Proof: (a) (Necessity). From Proposition 1 we know that $a_{i}>0, i=1,2$, whenever $\rho<1$. Combining this property with expressions given in (13), we have that, in equilibrium, the coefficients $c_{i}, i=1,2$, are strictly positive. Moreover, (14) and (15) can be rewritten as the expressions given in (21).

(Sufficiency). Suppose that the candidates equilibrium coefficients $c_{1}$ and $c_{2}$ are positive and satisfy (21). Then, the ratio $z=c_{1} / c_{2}>0$ and satisfies (20). Then, we conclude that an equilibrium exists and it is unique since we know that (20) has a unique positive solution. Finally, substituting this value of $z$ in the expressions stated in Lemma A2, we obtain the equilibrium coefficients of the demand functions.

(b) When $\rho=0$ or $\sigma_{\varepsilon_{i}}^{2}=0, i=1,2$, the demand functions are given by

$$
X_{i}\left(s_{i}, p\right)=\left(\mathbb{E}\left[\theta_{i} \mid s_{i}\right]-p\right) /\left(d_{i}+\lambda_{i}\right), i=1,2
$$

while when $\sigma_{\varepsilon_{i}}^{2}=\infty, i=1,2$, the demand functions hold

$$
X_{i}\left(s_{i}, p\right)=\left(\bar{\theta}_{i}-p\right) /\left(d_{i}+\lambda_{i}\right), i=1,2 .
$$


Moreover recall that the SOCs imply $d_{i}+\lambda_{i}>0$. Moreover, in all these cases we can express the coefficients of the demand functions in terms of $d_{i}, i=1,2$. In particular, $c_{i}=1 /\left(d_{i}+\lambda_{i}\right)>0$, $i=1,2$. Given our expression for $d_{i}$, we characterize $d_{i}, i=1,2$, as the positive solutions of the following system of equations:

$$
d_{i}=\left(\frac{n_{i}-1}{d_{i}+\lambda_{i}}+\frac{n_{j}}{d_{j}+\lambda_{j}}\right)^{-1} \text { for } i, j=1,2 \text { and } j \neq i .
$$

After some algebra, we conclude that this system has positive solutions if and only if $n_{1}+n_{2} \geq 3$. (c.1) (Necessity). Let $\bar{z}_{N}$ and $\bar{z}_{D}$ denote the highest root of $q_{N}(Z)$ and $q_{D}(Z)$, respectively. Notice that the positiveness of $c_{i}, i=1,2$, is equivalent to $\bar{z}_{N}>z>\bar{z}_{D}$. Therefore, $\bar{z}_{N}>\bar{z}_{D}$.

(Sufficiency). Suppose that $\bar{z}_{N}>\bar{z}_{D}$. Recall that Lemma A2 shows that there exists a unique positive value of $z$ that solves (20), which can be rewritten as

$$
\frac{\lambda_{1}}{\lambda_{2}}=\frac{n_{1}\left(n_{2}-1+n_{1} z\right) q_{N}(z)}{\left(n_{2}+\left(n_{1}-1\right) z\right) n_{2} q_{D}(z)} .
$$

This implies that $\bar{z}_{N}>z>\bar{z}_{D}$. Notice that these inequalities guarantee the positiveness of $c_{i}$, $i=1,2$.

(c.2) Suppose that $\rho \sigma_{\varepsilon_{1}}^{2}>0$ and $\sigma_{\varepsilon_{2}}^{2}=0$. In this case $\Xi_{2} \Delta_{2}^{-1}=1$ and, hence, $q_{D}(Z)=$ $Z n_{1}\left(n_{2}+Z n_{1}-2\right)$. On the one hand, if $n_{2}=1$, then $\bar{z}_{D}=1 / n_{1}$. As in c.1) the condition that guarantees the existence of equilibrium is $\bar{z}_{N}>\bar{z}_{D}$, which is equivalent to $n_{1}\left(2 \Xi_{1} \Delta_{1}^{-1}-1\right)>$ $\Xi_{1} \Delta_{1}^{-1}$, i.e., $\Xi_{1} \Delta_{1}^{-1}>1 / 2$ and $n_{1}>\Xi_{1} \Delta_{1}^{-1} /\left(2 \Xi_{1} \Delta_{1}^{-1}-1\right)$ or, using the expressions of $\Xi_{1}$ and $\Delta_{1}, 1-\rho^{2}+(1-2 \rho) \widehat{\sigma}_{\varepsilon_{1}}^{2}>0$ and $n_{1}>1+\widehat{\sigma}_{\varepsilon_{1}}^{2} \rho /\left(1-\rho^{2}+(1-2 \rho) \widehat{\sigma}_{\varepsilon_{1}}^{2}\right)$, which implies when $\rho$ is low enough and $n_{1}$ is large enough.

On the other hand, if $n_{2} \geq 2, q_{D}(Z)>0$ for all $Z>0$ and, therefore, we have that $c_{2}>0$ is satisfied. The positiveness of $c_{1}$ requires that $\bar{z}_{N}>z$. But, this inequality holds since $z$ solves Equation (22). To sum up, when $\sigma_{\varepsilon_{2}}^{2}=0$, an equilibrium exists if $n_{2}=1, n_{1}$ large enough and $\rho$ low enough, or if $n_{2} \geq 2$.

Now, suppose that $\rho \sigma_{\varepsilon_{2}}^{2}>0$ and $\sigma_{\varepsilon_{1}}^{2}=0$. In this case $\Xi_{1} \Delta_{1}^{-1}=1$ and, hence, $q_{N}(Z)=$ $n_{2}^{2}+n_{2}\left(n_{1}-2\right) Z$. On the one hand, if $n_{1}=1$, then $\bar{z}_{N}=n_{2}$. As in c.1) the condition that guarantees the existence of equilibrium is $\bar{z}_{N}>\bar{z}_{D}$, which is equivalent to $n_{2}\left(2 \Xi_{2} \Delta_{2}^{-1}-1\right)>$ $\Xi_{2} \Delta_{2}^{-1}$, i.e., $\Xi_{2} \Delta_{2}^{-1}>1 / 2$ and $n_{2}>\Xi_{2} \Delta_{2}^{-1} /\left(2 \Xi_{2} \Delta_{2}^{-1}-1\right)$ or, using the expressions of $\Xi_{2}$ and $\Delta_{2}, 1-\rho^{2}+(1-2 \rho) \widehat{\sigma}_{\varepsilon_{2}}^{2}>0$ and $n_{2}>1+\widehat{\sigma}_{\varepsilon_{2}}^{2} \rho /\left(1-\rho^{2}+(1-2 \rho) \widehat{\sigma}_{\varepsilon_{2}}^{2}\right)$, which implies when $\rho$ is low enough and $n_{2}$ is large enough.

On the other hand, if $n_{1} \geq 2, q_{N}(Z)>0$ for all $Z>0$ and, therefore, we have that $c_{1}>0$ is satisfied. The positiveness of $c_{2}$ requires that $z>\bar{z}_{D}$. But, this inequality holds since the equilibrium value, $z$, solves Equation (22). To sum up, when $\sigma_{\varepsilon_{1}}^{2}=0$, an equilibrium exists if $n_{1}=1, n_{2}$ large enough and $\rho$ low enough, or if $n_{1} \geq 2$. 
Lemma 2A. The condition $\bar{z}_{N}>\bar{z}_{D}$ given in the statement of Proposition $2 A$ is satisfied in the following cases:

(i) if $\rho<1$ and $n_{1}, n_{2}$ are large enough;

(ii) given $n_{i}, n_{j}$ is large enough and $\rho$ low enough, for $i, j=1,2$ and $j \neq i$.

Proof: We distinguish two cases: $n_{1}>1$ and $n_{1}=1$.

Case 1: $n_{1}>1$. In this case

$\bar{z}_{N}=\frac{n_{2}\left(\left(n_{1}-1\right)\left(2 \Xi_{1} \Delta_{1}^{-1}-1\right)-\left(2-\Xi_{1} \Delta_{1}^{-1}\right)+\sqrt{\left(2-\Xi_{1} \Delta_{1}^{-1}\right)^{2}+\left(n_{1}-1\right)\left(n_{1}+3-6 \Xi_{1} \Delta_{1}^{-1}\right)}\right)}{2 n_{1}\left(n_{1}-1\right)\left(1-\Xi_{1} \Delta_{1}^{-1}\right)}$

and

$$
\bar{z}_{D}=\frac{n_{2}+1-\Xi_{2} \Delta_{2}^{-1}\left(2 n_{2}-1\right)+\sqrt{\left(2-\Xi_{2} \Delta_{2}^{-1}\right)^{2}+\left(n_{2}-1\right)\left(n_{2}+3-6 \Xi_{2} \Delta_{2}^{-1}\right)}}{2 \Xi_{2} \Delta_{2}^{-1} n_{1}} .
$$

Proposition 2A indicates that an equilibrium exists if and only if $\bar{z}_{N}>\bar{z}_{D}$, or equivalently, $n_{1} \bar{z}_{N} / n_{2}>n_{1} \bar{z}_{D} / n_{2}$. Using the expressions of $\bar{z}_{N}$ and $\bar{z}_{D}$, we have that $n_{1} \bar{z}_{N} / n_{2}$ is increasing in $n_{1}$ and $n_{1} \bar{z}_{D} / n_{2}$ is decreasing in $n_{2}$. Taking limits, it follows that

$$
\lim _{n_{1} \rightarrow \infty} n_{1} \bar{z}_{N} / n_{2}=\Xi_{1} \Delta_{1}^{-1} /\left(1-\Xi_{1} \Delta_{1}^{-1}\right) \text { and } \lim _{n_{2} \rightarrow \infty} n_{1} \bar{z}_{D} / n_{2}=\left(1-\Xi_{2} \Delta_{2}^{-1}\right) /\left(\Xi_{2} \Delta_{2}^{-1}\right) .
$$

Moreover, using the expressions of $\Xi_{i}$ and $\Delta_{i}, i=1,2$, we have that

$$
\frac{\Xi_{1} \Delta_{1}^{-1}}{1-\Xi_{1} \Delta_{1}^{-1}}-\frac{1-\Xi_{2} \Delta_{2}^{-1}}{\Xi_{2} \Delta_{2}^{-1}}=\frac{\left(1-\rho^{2}\right)\left(1+\rho+\widehat{\sigma}_{\varepsilon_{1}}^{2}\right)\left(\left(1+\widehat{\sigma}_{\varepsilon_{1}}^{2}\right)\left(1+\widehat{\sigma}_{\varepsilon_{2}}^{2}\right)-\rho^{2}\right)}{\rho \widehat{\sigma}_{\varepsilon_{1}}^{2}\left(1+\rho+\widehat{\sigma}_{\varepsilon_{2}}^{2}\right)\left(1-\rho^{2}+\widehat{\sigma}_{\varepsilon_{1}}^{2}\right)}>0 .
$$

Hence, we get that, as $\rho<1, \lim _{n_{1} \rightarrow \infty} n_{1} \bar{z}_{N} / n_{2}>\lim _{n_{2} \rightarrow \infty} n_{1} \bar{z}_{D} / n_{2}$. This implies that whenever $\rho<1$ and $n_{1}$ and $n_{2}$ large enough, the existence of the equilibrium is guaranteed.

Consider now a fixed positive integer $n_{1}$, such that $n_{1}>1$. Using the fact that $\bar{z}_{N}$ is the positive root of $q_{N}(Z)$, it follow that $n_{1} \bar{z}_{N} / n_{2}>\Xi_{1} \Delta_{1}^{-1} /\left(2-\Xi_{1} \Delta_{1}^{-1}\right)$. Moreover,

$$
\Xi_{1} \Delta_{1}^{-1} /\left(2-\Xi_{1} \Delta_{1}^{-1}\right)>\left(1-\Xi_{2} \Delta_{2}^{-1}\right) /\left(\Xi_{2} \Delta_{2}^{-1}\right),
$$

whenever $\rho$ is low enough. Therefore,

$$
n_{1} \bar{z}_{N} / n_{2}>\Xi_{1} \Delta_{1}^{-1} /\left(2-\Xi_{1} \Delta_{1}^{-1}\right)>\left(1-\Xi_{2} \Delta_{2}^{-1}\right) /\left(\Xi_{2} \Delta_{2}^{-1}\right)=\lim _{n_{2} \rightarrow \infty} n_{1} \bar{z}_{D} / n_{2} .
$$

Hence, we conclude that if $n_{2}$ is large enough, as $n_{1} \bar{z}_{D} / n_{2}$ is decreasing in $n_{2}$, the previous inequalities imply that $n_{1} \bar{z}_{N} / n_{2}>n_{1} \bar{z}_{D} / n_{2}$ or, equivalently, $\bar{z}_{N}>\bar{z}_{D}$. Applying Proposition $2 \mathrm{~A}$, it follows that in this case there exists an equilibrium provided that $n_{2}$ is high enough and $\rho$ low enough. 
Consider now a fixed positive integer $n_{2}$, such that $n_{2} \geq 1$, and assume again that $\rho<1$. Using the fact that $\bar{z}_{D}$ is the positive root of $q_{D}(Z)$, it follow that $n_{1} \bar{z}_{D} / n_{2} \leq\left(2-\Xi_{2} \Delta_{2}^{-1}\right) /\left(\Xi_{2} \Delta_{2}^{-1}\right)$. In addition, when $\rho$ is low enough, then we have that

$$
\left(2-\Xi_{2} \Delta_{2}^{-1}\right) /\left(\Xi_{2} \Delta_{2}^{-1}\right)<\Xi_{1} \Delta_{1}^{-1} /\left(1-\Xi_{1} \Delta_{1}^{-1}\right)=\lim _{n_{1} \rightarrow \infty} n_{1} \bar{z}_{N} / n_{2}
$$

Thus, we have that $n_{1} \bar{z}_{D} / n_{2}<\lim _{n_{1} \rightarrow \infty} n_{1} \bar{z}_{N} / n_{2}$. Using the fact that $n_{1} \bar{z}_{N} / n_{2}$ increases with $n_{1}$, we have that, when $n_{1}$ is high enough, $n_{1} \bar{z}_{D} / n_{2}<n_{1} \bar{z}_{N} / n_{2}$ or, equivalently, $\bar{z}_{D}<\bar{z}_{N}$, which guarantees the existence of equilibrium. To sum up, we have that given $n_{2}$, there exists an equilibrium provided that $n_{1}$ is high enough and $\rho$ low enough.

Case 2: $n_{1}=1$. In this case, we have that $\bar{z}_{N}=n_{2} \Xi_{1} \Delta_{1}^{-1} /\left(2-\Xi_{1} \Delta_{1}^{-1}\right)$ and

$$
\bar{z}_{D}=\frac{n_{2}+1-\Xi_{2} \Delta_{2}^{-1}\left(2 n_{2}-1\right)+\sqrt{\left(2-\Xi_{2} \Delta_{2}^{-1}\right)^{2}+\left(n_{2}-1\right)\left(n_{2}+3-6 \Xi_{2} \Delta_{2}^{-1}\right)}}{2 \Xi_{2} \Delta_{2}^{-1}} .
$$

Furthermore, whenever $\rho$ is low enough, (25) holds. Therefore, it follows that

$$
\bar{z}_{N} / n_{2}=\Xi_{1} \Delta_{1}^{-1} /\left(2-\Xi_{1} \Delta_{1}^{-1}\right)>\left(1-\Xi_{2} \Delta_{2}^{-1}\right) /\left(\Xi_{2} \Delta_{2}^{-1}\right)=\lim _{n_{2} \rightarrow \infty} \bar{z}_{D} / n_{2}
$$

Using the fact that $\bar{z}_{D} / n_{2}$ decreases with $n_{2}$, the previous inequality implies that $\bar{z}_{N} / n_{2}>\bar{z}_{D} / n_{2}$ whenever $n_{2}$ is high enough, i.e., $\bar{z}_{N}>\bar{z}_{D}$, which guarantees the existence of equilibrium. To sum up, we have that when $n_{1}=1$, there exists an equilibrium provided that $n_{2}$ is high enough and $\rho$ low enough.

Proof of Proposition 2: This proposition directly follows from Proposition 2A and Lemma $2 \mathrm{~A}$.

Remark (symmetric groups). Let $n_{i}=n, \lambda_{i}=\lambda$, and $\sigma_{\varepsilon_{i}}^{2}=\sigma_{\varepsilon}^{2}, i=1,2$. Here $z=1$ in equilibrium. From Proposition $2 \mathrm{~A}$ we know that, if an equilibrium exists, then the value of $z$ is in the interval $\left(\bar{z}_{D}, \bar{z}_{N}\right)$. It follows that $\bar{z}_{N}>1>\bar{z}_{D}$ or, equivalently, that $q_{N}(1)>0$ and $q_{D}(1)>0$. After performing some algebra, we find that the foregoing inequalities are satisfied iff $n>1+\rho \widehat{\sigma}_{\varepsilon}^{2} /\left((1-\rho)\left(1+\rho+\widehat{\sigma}_{\varepsilon}^{2}\right)\right)$, where $\widehat{\sigma}_{\varepsilon}^{2}=\sigma_{\varepsilon}^{2} / \sigma_{\theta}^{2}$.

Proof of Proposition 3: Let $\rho \sigma_{\varepsilon_{1}}^{2} \sigma_{\varepsilon_{2}}^{2}$. In what follows we prove the following comparative statics results for $i, j=1,2, i \neq j$ :

(a) $\partial b_{i} / \partial \bar{\theta}_{i}>0, \partial a_{i} / \partial \bar{\theta}_{i}=0$, and $\partial c_{i} / \partial \bar{\theta}_{i}=0$,

(b) $\partial b_{i} / \partial \bar{\theta}_{j}<0, \partial a_{i} / \partial \bar{\theta}_{j}=0$, and $\partial c_{i} / \partial \bar{\theta}_{j}=0$,

(c) $\partial b_{i} / \partial Q>0, \partial a_{i} / \partial Q=0$, and $\partial c_{i} / \partial Q=0$,

(d) $\partial a_{i} / \partial \lambda_{i}<0$ and $\partial c_{i} / \partial \lambda_{i}<0$ 
(e) $\partial a_{i} / \partial \lambda_{j}<0$ and $\partial c_{i} / \partial \lambda_{j}<0$,

(f) $\partial a_{i} / \partial \rho<0$ and $\partial c_{i} / \partial \rho<0$,

(g) $\partial\left(d_{i} / d_{j}\right) / \partial \sigma_{\varepsilon_{i}}^{2}<0, \partial\left(d_{i} / d_{j}\right) / \partial \sigma_{\varepsilon_{j}}^{2}>0, \partial\left(d_{i} / d_{j}\right) / \partial \lambda_{i}<0$, and $\partial\left(d_{i} / d_{j}\right) / \partial \lambda_{j}>0$,

(h) $\partial a_{i} / \partial \sigma_{\varepsilon_{i}}^{2}<0$ and $\partial c_{i} / \partial \sigma_{\varepsilon_{i}}^{2}<0$,

(i) $\partial a_{i} / \partial \sigma_{\varepsilon_{j}}^{2}<0$ and $\partial c_{i} / \partial \sigma_{\varepsilon_{j}}^{2}<0$, and

(j) $\partial d_{i} / \partial n_{i}<0$ and $\partial d_{j} / \partial n_{i}<0$.

From Lemma A1, we know that the equilibrium coefficients that depend on $\bar{\theta}_{i}, \bar{\theta}_{j}$ and $Q$ are $b_{1}$ and $b_{2}$. Using Lemma A2 and after some algebra, the results given in (a), (b) and (c) are obtained. In what follows, without any loss of generality, let $i=1$. First, we prove that $\partial z / \partial \lambda_{1}<0$. From Lemma A2, we know that $z$ is the unique positive solution of the following equation:

$$
\frac{\lambda_{1}}{\lambda_{2}}-\frac{N(z)}{D(z)}=0
$$

where

$$
\begin{aligned}
& N(z)=\Xi_{1} \Delta_{1}^{-1}-n_{1}\left(1-\Xi_{1} \Delta_{1}^{-1}\right) z / n_{2}-z\left(\left(n_{1}-1\right) z+n_{2}\right)^{-1} \text { and } \\
& D(z)=\Xi_{2} \Delta_{2}^{-1} z-n_{2}\left(1-\Xi_{2} \Delta_{2}^{-1}\right) / n_{1}-z\left(n_{1} z+n_{2}-1\right)^{-1},
\end{aligned}
$$

with $\Xi_{i} \Delta_{i}^{-1}=\left(1-\rho^{2}+\widehat{\sigma}_{\varepsilon_{j}}^{2}\right)\left(1+\rho+\widehat{\sigma}_{\varepsilon_{i}}^{2}\right)\left(\left(\left(1+\widehat{\sigma}_{\varepsilon_{i}}^{2}\right)\left(1+\widehat{\sigma}_{\varepsilon_{j}}^{2}\right)-\rho^{2}\right)(1+\rho)\right)^{-1}, i, j=1,2, i \neq$ $j$. Applying the Implicit Function Theorem,

$$
\frac{\partial z}{\partial \lambda_{i}}=-\frac{\partial\left(\lambda_{1} / \lambda_{2}-N(z) / D(z)\right) / \partial \lambda_{i}}{\partial\left(\lambda_{1} / \lambda_{2}-N(z) / D(z)\right) / \partial z}, i=1,2 .
$$

As $\partial\left(\lambda_{1} / \lambda_{2}-N(z) / D(z)\right) / \partial \lambda_{1}>0, \partial\left(\lambda_{1} / \lambda_{2}-N(z) / D(z)\right) / \partial \lambda_{2}<0$, and

$$
\frac{\partial\left(\lambda_{1} / \lambda_{2}-N(z) / D(z)\right)}{\partial z}>0,
$$

because of $z \in\left(\bar{z}_{D}, \bar{z}_{N}\right)$, we conclude that $\partial z / \partial \lambda_{1}<0$ and $\partial z / \partial \lambda_{2}>0$.

Next, we study the relationship between $c^{\prime} s$ and $\lambda_{1}$. Differentiating (15), we have

$$
\frac{\partial c_{2}}{\partial \lambda_{1}}=\frac{\partial c_{2}}{\partial z} \frac{\partial z}{\partial \lambda_{1}}=\frac{1}{\lambda_{2}}\left(\frac{n_{2}\left(1-\Xi_{2} \Delta_{2}^{-1}\right)}{n_{1} z^{2}}+\frac{n_{1}}{\left(n_{1} z+n_{2}-1\right)^{2}}\right) \frac{\partial z}{\partial \lambda_{1}}<0,
$$

since $\partial z / \partial \lambda_{1}<0$. Moreover, as $c_{1}=z c_{2}$, it follows that $\partial c_{1} / \partial \lambda_{1}=\left(\partial z / \partial \lambda_{1}\right) c_{2}+z\left(\partial c_{2} / \partial \lambda_{1}\right)<$ 0 , because of the positiveness of $c_{2}$ and $z$, and the negativeness of $\partial z / \partial \lambda_{1}$ and $\partial c_{2} / \partial \lambda_{1}$. In relation to $a_{1}$ and $a_{2}$, from (13), direct computation yields $\partial a_{1} / \partial \lambda_{1}<0$ and $\partial a_{2} / \partial \lambda_{1}<0$, since $\partial c_{1} / \partial \lambda_{1}<0$ and $\partial c_{2} / \partial \lambda_{1}<0$. 
Now, we study how the correlation coefficient $\rho$ affects $a_{1}$. Let $y=a_{1} / a_{2}$. As $a_{1}=\Delta_{1} c_{1}$ and $a_{2}=\Delta_{2} c_{2}$, then $z=\Delta_{2} y / \Delta_{1}$. Substituting this expression in (20), and after some algebra, we have that

$$
\frac{\lambda_{1}}{\lambda_{2}} y=\frac{\widetilde{N}(y, \rho)}{\widetilde{D}(y, \rho)}
$$

where $\tilde{N}(y, \rho)=\frac{1-\rho^{2}+\widehat{\sigma}_{\varepsilon_{2}}^{2}-\frac{n_{1}}{n_{2}} \widehat{\sigma}_{\varepsilon_{1}}^{2} \rho y}{\left(1+\widehat{\sigma}_{\varepsilon_{1}}^{2}\right)\left(1+\widehat{\sigma}_{\varepsilon_{2}}^{2}\right)-\rho^{2}}-\left(\left(n_{1}-1\right) \frac{1+\rho+\widehat{\sigma}_{\varepsilon_{1}}^{2}}{1+\rho}+n_{2} \frac{1+\rho+\widehat{\sigma}_{\varepsilon_{2}}^{2}}{1+\rho} \frac{1}{y}\right)^{-1}$ and

$$
\widetilde{D}(y, \rho)=\frac{1-\rho^{2}+\widehat{\sigma}_{\varepsilon_{1}}^{2}-\frac{n_{2}}{n_{1}} \widehat{\sigma}_{\varepsilon_{2}}^{2} \rho \frac{1}{y}}{\left(1+\widehat{\sigma}_{\varepsilon_{1}}^{2}\right)\left(1+\widehat{\sigma}_{\varepsilon_{2}}^{2}\right)-\rho^{2}}-\left(n_{1} \frac{1+\rho+\widehat{\sigma}_{\varepsilon_{1}}^{2}}{1+\rho} y+\left(n_{2}-1\right) \frac{1+\rho+\widehat{\sigma}_{\varepsilon_{2}}^{2}}{1+\rho}\right)^{-1} .
$$

Moreover, $a_{1}=\widetilde{N}(y, \rho) / \lambda_{1}$ and $a_{2}=\widetilde{D}(y, \rho) / \lambda_{2}$. Hence,

$$
\frac{\partial a_{1}}{\partial \rho}=\frac{(\partial \tilde{N}(y, \rho) / \partial y)(\partial y / \partial \rho)+\partial \tilde{N}(y, \rho) / \partial \rho}{\lambda_{1}} .
$$

Thus, in order to show $\partial a_{1} / \partial \rho<0$, it suffices to prove that

$$
\frac{\partial \widetilde{N}(y, \rho)}{\partial y} \frac{\partial y}{\partial \rho}+\frac{\partial \widetilde{N}(y, \rho)}{\partial \rho}<0
$$

Direct computation yields $\partial \widetilde{N}(y, \rho) / \partial y<0$. Then, $(29)$ is equivalent to

$$
\frac{\partial y}{\partial \rho}>-\frac{\partial \widetilde{N}(y, \rho) / \partial \rho}{\partial \widetilde{N}(y, \rho) / \partial y}
$$

Moreover, recall that $y$ in equilibrium is the unique positive value that satisfies (28). Thus, applying the Implicit Function Theorem, it follows that

$$
\frac{\partial y}{\partial \rho}=-\frac{\partial\left(\lambda_{1} y / \lambda_{2}-\tilde{N}(y, \rho) / \widetilde{D}(y, \rho)\right) / \partial \rho}{\partial\left(\lambda_{1} y / \lambda_{2}-\widetilde{N}(y, \rho) / \widetilde{D}(y, \rho)\right) / \partial y} .
$$

Then, (30) can be rewritten as

$$
-\frac{\partial\left(\lambda_{1} y / \lambda_{2}-\tilde{N}(y, \rho) / \widetilde{D}(y, \rho)\right) / \partial \rho}{\partial\left(\lambda_{1} y / \lambda_{2}-\tilde{N}(y, \rho) / \widetilde{D}(y, \rho)\right) / \partial y}>-\frac{\partial \widetilde{N}(y, \rho) / \partial \rho}{\partial \widetilde{N}(y, \rho) / \partial y},
$$

or using the fact that in equilibrium $\partial\left(\lambda_{1} y / \lambda_{2}-\widetilde{N}(y, \rho) / \widetilde{D}(y, \rho)\right) / \partial y>0,(30)$ is satisfied iff

$$
-\frac{\partial\left(\lambda_{1} y / \lambda_{2}-\tilde{N}(y, \rho) / \widetilde{D}(y, \rho)\right)}{\partial \rho}>-\left(\frac{\partial \widetilde{N}(y, \rho) / \partial \rho}{\partial \widetilde{N}(y, \rho) / \partial y}\right) \frac{\partial\left(\lambda_{1} y / \lambda_{2}-\tilde{N}(y, \rho) / \widetilde{D}(y, \rho)\right)}{\partial y} .
$$


Notice that

$$
-\frac{\partial\left(\lambda_{1} y / \lambda_{2}-\widetilde{N}(y, \rho) / \widetilde{D}(y, \rho)\right)}{\partial \rho}=-\frac{(\partial \widetilde{N}(y, \rho) / \partial \rho) \widetilde{D}(y, \rho)-\widetilde{N}(y, \rho)(\partial \widetilde{D}(y, \rho) / \partial \rho)}{\widetilde{D}^{2}(y, \rho)}
$$

or using (26),

$$
-\frac{\partial\left(\lambda_{1} y / \lambda_{2}-\widetilde{N}(y, \rho) / \widetilde{D}(y, \rho)\right)}{\partial \rho}=-\frac{\partial \widetilde{N}(y, \rho) / \partial \rho-\lambda_{1} y(\partial \widetilde{D}(y, \rho) / \partial \rho) / \lambda_{2}}{\widetilde{D}(y, \rho)} .
$$

Analogously,

$$
\frac{\partial\left(\lambda_{1} y / \lambda_{2}-\widetilde{N}(y, \rho) / \widetilde{D}(y, \rho)\right)}{\partial y}=\frac{\lambda_{1}}{\lambda_{2}}-\frac{\partial \widetilde{N}(y, \rho) / \partial y-\lambda_{1} y(\partial \widetilde{D}(y, \rho) / \partial y) / \lambda_{2}}{\widetilde{D}(y, \rho)} .
$$

Therefore, (31) is equivalent to

$$
\begin{aligned}
\frac{\partial \widetilde{N}(y, \rho) / \partial \rho-\lambda_{1} y(\partial \widetilde{D}(y, \rho) / \partial \rho) / \lambda_{2}}{\widetilde{D}(y, \rho)}> & -\frac{\partial \widetilde{N}(y, \rho) / \partial \rho}{\partial \widetilde{N}(y, \rho) / \partial y} \times \\
& \left(\frac{\lambda_{1}}{\lambda_{2}}-\frac{\partial \widetilde{N}(y, \rho) / \partial y-\lambda_{1} y(\partial \widetilde{D}(y, \rho) / \partial y) / \lambda_{2}}{\widetilde{D}(y, \rho)}\right),
\end{aligned}
$$

or,

$$
-\frac{y(\partial \widetilde{D}(y, \rho) / \partial \rho)}{\widetilde{D}(y, \rho)}>-\frac{\partial \widetilde{N}(y, \rho) / \partial \rho}{\partial \widetilde{N}(y, \rho) / \partial y}\left(1+\frac{y(\partial \widetilde{D}(y, \rho) / \partial y)}{\widetilde{D}(y, \rho)}\right) .
$$

Moreover, recall that $a_{2}=\widetilde{D}(y, \rho) / \lambda_{2}$. The positiveness of $a_{2}$ tells us that $\widetilde{D}(y, \rho)>0$. After some algebra, we have that $\partial \widetilde{D}(y, \rho) / \partial \rho<0, \partial \widetilde{N}(y, \rho) / \partial \rho<0$ and $\partial \widetilde{D}(y, \rho) / \partial y>0$. Hence, we conclude that the left-hand side $(L H S)$ of (32) is positive, whereas the right-hand side $(R H S)$ of $(32)$ is negative since $\partial \widetilde{N}(y, \rho) / \partial y<0$. Consequently, the fact that $(32)$ is satisfied allows us to conclude that $\partial a_{1} / \partial \rho<0$.

Concerning the effect of $\rho$ on $c_{1}$, recall that $c_{1}=a_{1} / \Delta_{1}=\left(1+\rho+\widehat{\sigma}_{\varepsilon_{1}}^{2}\right) a_{1} /(1+\rho)$. This expression tells us that $c_{1}$ is the product of two decreasing positive functions in $\rho$. Therefore, $\partial c_{1} / \partial \rho<0$.

Next, we prove that $\partial\left(d_{1} / d_{2}\right) / \partial \sigma_{\varepsilon_{1}}^{2}<0$ and $\partial\left(d_{1} / d_{2}\right) / \partial \sigma_{\varepsilon_{2}}^{2}>0$. From the expressions of $d_{1}, d_{2}$, and $z$, it follows that $d_{1} / d_{2}=\left(n_{1} z+\left(n_{2}-1\right)\right) /\left(\left(n_{1}-1\right) z+n_{2}\right)$. Applying the chain rule, we get $\partial\left(d_{1} / d_{2}\right) / \partial \sigma_{\varepsilon_{i}}^{2}=\left(\partial\left(d_{1} / d_{2}\right) / \partial z\right)\left(\partial z / \partial \sigma_{\varepsilon_{i}}^{2}\right)$. As $\partial\left(d_{1} / d_{2}\right) / \partial z>0$, we know that 
the sign of $\partial\left(d_{1} / d_{2}\right) / \partial \sigma_{\varepsilon_{i}}^{2}$ is the same as the sign of $\partial z / \partial \sigma_{\varepsilon_{i}}^{2}$. Applying the Implicit Function Theorem,

$$
\frac{\partial z}{\partial \sigma_{\varepsilon_{i}}^{2}}=-\frac{\partial\left(\lambda_{1} / \lambda_{2}-N(z) / D(z)\right) / \partial \sigma_{\varepsilon_{i}}^{2}}{\partial\left(\lambda_{1} / \lambda_{2}-N(z) / D(z)\right) / \partial z} .
$$

Using $\partial\left(\lambda_{1} / \lambda_{2}-N(z) / D(z)\right) / \partial \sigma_{\varepsilon_{1}}^{2}>0, \partial\left(\lambda_{1} / \lambda_{2}-N(z) / D(z)\right) / \partial \sigma_{\varepsilon_{2}}^{2}<0$, and the inequality given in (27), we obtain $\partial z / \partial \sigma_{\varepsilon_{1}}^{2}<0$ and $\partial z / \partial \sigma_{\varepsilon_{2}}^{2}>0$, and hence, we conclude that $\partial\left(d_{1} / d_{2}\right) / \partial \sigma_{\varepsilon_{1}}^{2}<0$ and $\partial\left(d_{1} / d_{2}\right) / \partial \sigma_{\varepsilon_{2}}^{2}>0$. Analogously, the negativeness of $\partial z / \partial \lambda_{1}$ and the positiveness of $\partial z / \partial \lambda_{2}$ allows us to conclude that $\partial\left(d_{1} / d_{2}\right) / \partial \lambda_{1}<0$ and $\partial\left(d_{1} / d_{2}\right) / \partial \lambda_{2}>0$.

Now, we study how $a_{1}$ and $c_{1}$ vary with a change in $\sigma_{\varepsilon_{i}}^{2}, i=1,2$. In order to do that first we analyze the effect of $\sigma_{\varepsilon_{i}}^{2}$ on $d_{1}$ and $d_{2}$. From Proposition 1, we know that $d_{i}=$ $\left(\left(n_{i}-1\right) c_{i}+n_{j} c_{j}\right)^{-1}$ and $a_{i}=\Delta_{i} c_{i}>0, i=1,2$. Therefore, $d_{i}=\left(\left(n_{i}-1\right) \Delta_{i}^{-1} a_{i}+n_{j} \Delta_{j}^{-1} a_{j}\right)^{-1}$, $i, j=1,2, j \neq i$. Substituting the expressions of (11) and the expression for $\Delta_{i}$ given in Lemma A2, it follows that

$$
d_{i}=\left(\frac{\left(n_{i}-1\right) n_{j}}{\Omega_{i}}+\frac{n_{j} n_{i}}{\Omega_{j}}\right)^{-1}
$$

where $\Omega_{i}=n_{j} \Upsilon_{i}\left(d_{i}+\lambda_{i}\right)+n_{i}\left(\Upsilon_{i}-1\right)\left(d_{j}+\lambda_{j}\right)$ and $\Omega_{j}=n_{i} \Upsilon_{j}\left(d_{j}+\lambda_{j}\right)+n_{j}\left(\Upsilon_{j}-1\right)\left(d_{i}+\lambda_{i}\right)$, with $\Upsilon_{i}=\Xi_{j} /\left(\Xi_{j}-\Psi_{i}\right)=\left(1-\rho^{2}+\widehat{\sigma}_{\varepsilon_{i}}^{2}\right) /\left((1-\rho)\left(1+\rho+\widehat{\sigma}_{\varepsilon_{i}}^{2}\right)\right)>1, i, j=1,2, j \neq i$. Therefore, we derive the following equations that are satisfied in equilibrium: $F_{i}\left(\sigma_{\varepsilon_{1}}^{2}, \sigma_{\varepsilon_{2}}^{2}, d_{1}, d_{2}\right)=0$, $i=1,2$, where

$$
F_{i}\left(\sigma_{\varepsilon_{1}}^{2}, \sigma_{\varepsilon_{2}}^{2}, d_{1}, d_{2}\right)=\frac{\left(n_{i}-1\right) n_{j} d_{i}}{\Omega_{i}}+\frac{n_{i} n_{j} d_{i}}{\Omega_{j}}-1,
$$

$i, j=1,2, j \neq i$. Let $D F_{d_{1}, d_{2}}\left(\sigma_{\varepsilon_{1}}^{2}, \sigma_{\varepsilon_{2}}^{2}, d_{1}, d_{2}\right)$ denote the following matrix:

$$
\left(\begin{array}{cc}
\partial F_{1}\left(\sigma_{\varepsilon_{1}}^{2}, \sigma_{\varepsilon_{2}}^{2}, d_{1}, d_{2}\right) / \partial d_{1} & \partial F_{1}\left(\sigma_{\varepsilon_{1}}^{2}, \sigma_{\varepsilon_{2}}^{2}, d_{1}, d_{2}\right) / \partial d_{2} \\
\partial F_{2}\left(\sigma_{\varepsilon_{1}}^{2}, \sigma_{\varepsilon_{2}}^{2}, d_{1}, d_{2}\right) / \partial d_{1} & \partial F_{2}\left(\sigma_{\varepsilon_{1}}^{2}, \sigma_{\varepsilon_{2}}^{2}, d_{1}, d_{2}\right) / \partial d_{2}
\end{array}\right) .
$$

After some tedious algebra, it can be shown that the determinant of $D F_{d_{1}, d_{2}}\left(\sigma_{\varepsilon_{1}}^{2}, \sigma_{\varepsilon_{2}}^{2}, d_{1}, d_{2}\right)$ is strictly positive. In particular, it is not null and, therefore, this matrix is invertible. Hence, we can apply the Implicit Function Theorem, we have

$$
\begin{aligned}
& \left(\begin{array}{ll}
\partial d_{1} / \partial \sigma_{\varepsilon_{1}}^{2} & \partial d_{1} / \partial \sigma_{\varepsilon_{2}}^{2} \\
\partial d_{2} / \partial \sigma_{\varepsilon_{1}}^{2} & \partial d_{2} / \partial \sigma_{\varepsilon_{1}}^{2}
\end{array}\right)= \\
& -\left(D F_{d_{1}, d_{2}}\left(\sigma_{\varepsilon_{1}}^{2}, \sigma_{\varepsilon_{2}}^{2}, d_{1}, d_{2}\right)\right)^{-1}\left(\begin{array}{cc}
\partial F_{1}\left(\sigma_{\varepsilon_{1}}^{2}, \sigma_{\varepsilon_{2}}^{2}, d_{1}, d_{2}\right) / \partial \sigma_{\varepsilon_{1}}^{2} & \partial F_{1}\left(\sigma_{\varepsilon_{1}}^{2}, \sigma_{\varepsilon_{2}}^{2}, d_{1}, d_{2}\right) / \partial \sigma_{\varepsilon_{2}}^{2} \\
\partial F_{2}\left(\sigma_{\varepsilon_{1}}^{2}, \sigma_{\varepsilon_{2}}^{2}, d_{1}, d_{2}\right) / \partial \sigma_{\varepsilon_{1}}^{2} & \partial F_{2}\left(\sigma_{\varepsilon_{1}}^{2}, \sigma_{\varepsilon_{2}}^{2}, d_{1}, d_{2}\right) / \partial \sigma_{\varepsilon_{2}}^{2}
\end{array}\right) .
\end{aligned}
$$

It is easy to see that all the elements of $\left(D F_{d_{1}, d_{2}}\left(\sigma_{\varepsilon_{1}}^{2}, \sigma_{\varepsilon_{2}}^{2}, d_{1}, d_{2}\right)\right)^{-1}$ are positive. Moreover, $\partial F_{i}\left(\sigma_{\varepsilon_{1}}^{2}, \sigma_{\varepsilon_{2}}^{2}, d_{1}, d_{2}\right) / \partial \sigma_{\varepsilon_{i}}^{2}<0$ and $\partial F_{i}\left(\sigma_{\varepsilon_{1}}^{2}, \sigma_{\varepsilon_{2}}^{2}, d_{1}, d_{2}\right) / \partial \sigma_{\varepsilon_{j}}^{2}<0, i, j=1,2, j \neq i$. Hence, (33) implies that $\partial d_{i} / \partial \sigma_{\varepsilon_{i}}^{2}>0$ and $\partial d_{i} / \partial \sigma_{\varepsilon_{j}}^{2}>0$. 
Next, we study the comparative statics of $c_{1}$ and $c_{2}$ with respect to $\sigma_{\varepsilon_{1}}^{2}$. Recall that $c_{i}=$ $n_{j} / \Omega_{i}, i, j=1,2, j \neq i$. Using the fact that $\Upsilon_{1}, d_{1}$, and $d_{2}$ are increasing in $\sigma_{\varepsilon_{1}}^{2}$ and that $\Upsilon_{2}$ is independent of $\sigma_{\varepsilon_{1}}^{2}$, we have that $\Omega_{1}$ and $\Omega_{2}$ are increasing in $\sigma_{\varepsilon_{1}}^{2}$, which allows us to conclude that $c_{1}$ and $c_{2}$ are decreasing in $\sigma_{\varepsilon_{1}}^{2}$. Combining these results with the fact that $\Delta_{1}$ is decreasing in $\sigma_{\varepsilon_{1}}^{2}$ and $\Delta_{2}$ is independent of $\sigma_{\varepsilon_{1}}^{2}$, it follows that $a_{1}$ and $a_{2}$ are decreasing in $\sigma_{\varepsilon_{1}}^{2}$, since $a_{1}=\Delta_{1} c_{1}$ and $a_{2}=\Delta_{2} c_{2}$.

Finally, concerning h), notice that doing a similar reasoning as before we derive the following equations that are satisfied in equilibrium: $F_{i}\left(n_{1}, n_{2}, d_{1}, d_{2}\right)=0, i=1,2$, where $F_{i}\left(n_{1}, n_{2}, d_{1}, d_{2}\right)=F_{i}\left(\sigma_{\varepsilon_{1}}^{2}, \sigma_{\varepsilon_{2}}^{2}, d_{1}, d_{2}\right), i, j=1,2, j \neq i$. Hence,

$$
\begin{aligned}
& \left(\begin{array}{ll}
\partial d_{1} / \partial n_{1} & \partial d_{1} / \partial n_{2} \\
\partial d_{2} / \partial n_{1} & \partial d_{2} / \partial n_{2}
\end{array}\right)= \\
& \quad-\left(D F_{d_{1}, d_{2}}\left(n_{1}, n_{2}, d_{1}, d_{2}\right)\right)^{-1}\left(\begin{array}{ll}
\partial F_{1}\left(n_{1}, n_{2}, d_{1}, d_{2}\right) / \partial n_{1} & \partial F_{1}\left(n_{1}, n_{2}, d_{1}, d_{2}\right) / \partial n_{2} \\
\partial F_{2}\left(n_{1}, n_{2}, d_{1}, d_{2}\right) / \partial n_{1} & \partial F_{2}\left(n_{1}, n_{2}, d_{1}, d_{2}\right) / \partial n_{2}
\end{array}\right) .
\end{aligned}
$$

Taking into account that all the elements of the previous two matrices are positive, we conclude that $\partial d_{i} / \partial n_{i}<0$ and $\partial d_{i} / \partial n_{j}<0, i, j=1,2, j \neq i$.

Proof of Corollary 2(i): Suppose that $\sigma_{\varepsilon_{1}}^{2} \geq \sigma_{\varepsilon_{2}}^{2}, \lambda_{1} \geq \lambda_{2}$, and $n_{1} \geq n_{2}$. Using the expressions of $\Xi_{i}$ and $\Delta_{i}, i=1,2$, it is easy to see that in this case $\Xi_{2} \Delta_{2}^{-1}>\Xi_{1} \Delta_{1}^{-1}$. Next, we distinguish two cases:

Case 1: $\left(n_{1}+n_{2}-2\right) n_{1} /\left(\left(n_{1}+n_{2}\right)\left(n_{1}+n_{2}-1\right)\right) \geq 1-\Xi_{2} \Delta_{2}^{-1}$. Evaluating the polynomial $g(z)$, stated in the proof of Lemma A2, at $z=1$, we have that in this case $g(1) \geq 0$. This implies that $z \leq 1$, and therefore, $c_{1} \leq c_{2}$. In addition, using the expressions of $d_{1}$ and $d_{2}$, we get $\operatorname{sgn}\left\{d_{1}-d_{2}\right\}=\operatorname{sgn}\left\{c_{1}-c_{2}\right\}$, which implies $d_{1} \leq d_{2}$. Finally, notice that $\Delta_{1} \leq \Delta_{2}$ whenever $\sigma_{\varepsilon_{1}}^{2} \geq \sigma_{\varepsilon_{2}}^{2}$. Hence, $a_{1} / a_{2}=z \Delta_{1} / \Delta_{2} \leq 1$.

Case 2: $\left(n_{1}+n_{2}-2\right) n_{1} /\left(\left(n_{1}+n_{2}\right)\left(n_{1}+n_{2}-1\right)\right)<1-\Xi_{2} \Delta_{2}^{-1}$. Notice that

$$
\frac{\left(n_{1}+n_{2}-2\right) n_{2}}{\left(n_{1}+n_{2}\right)\left(n_{1}+n_{2}-1\right)}-\left(1-\Xi_{1} \Delta_{1}^{-1}\right) \leq \frac{\left(n_{1}+n_{2}-2\right) n_{1}}{\left(n_{1}+n_{2}\right)\left(n_{1}+n_{2}-1\right)}-\left(1-\Xi_{2} \Delta_{2}^{-1}\right),
$$

since $\Xi_{2} \Delta_{2}^{-1}>\Xi_{1} \Delta_{1}^{-1}$ and $n_{1} \geq n_{2}$. Thus, in this case we have that $q_{N}(1)<0$ and $q_{D}(1)<0$. Taking into account the shape of these polynomials, the previous two inequalities imply that $\bar{z}_{D}>1>\bar{z}_{N}$. However, Proposition $2 \mathrm{~A}$ indicates that in this case there is no equilibrium.

Corollary 2A(ii). Suppose that $\sigma_{\varepsilon_{1}}^{2} \geq \sigma_{\varepsilon_{2}}^{2}, \lambda_{1} \geq \lambda_{2}$, and $n_{1} \geq n_{2}$, and suppose that at least one of these inequalities is strict. If

$$
(1-\rho) n_{1}\left(\frac{n_{2}\left(1+\rho+\widehat{\sigma}_{\varepsilon_{1}}^{2}\right)}{n_{2}\left(1-\rho^{2}+\widehat{\sigma}_{\varepsilon_{1}}^{2}\right)+n_{1} \rho \widehat{\sigma}_{\varepsilon_{1}}^{2}}+\frac{\left(n_{2}-1\right)\left(1+\rho+\widehat{\sigma}_{\varepsilon_{2}}^{2}\right)}{n_{1}\left(1-\rho^{2}+\widehat{\sigma}_{\varepsilon_{2}}^{2}\right)+n_{2} \rho \widehat{\sigma}_{\varepsilon_{2}}^{2}}\right) \leq 1,
$$


then $d_{1}+\lambda_{1}<d_{2}+\lambda_{2}$ always holds. Otherwise, $d_{1}+\lambda_{1}>d_{2}+\lambda_{2}$ iff $\lambda_{1} / \lambda_{2}$ is high enough.

Proof: By virtue of (16), the inequality $d_{1}+\lambda_{1}>d_{2}+\lambda_{2}$ is equivalent to

$$
\left(\Xi_{1}-\frac{n_{1}}{n_{2}} \frac{a_{1}}{a_{2}} \Psi_{1}\right) / a_{1}>\left(\Xi_{2}-\frac{n_{2}}{n_{1}} \frac{a_{2}}{a_{1}} \Psi_{2}\right) / a_{2}
$$

Using (13) and (19), and after some algebra, the previous inequality is equivalent to

$$
z<\widehat{z}
$$

where $\widehat{z}=\left(\Xi_{1} \Delta_{1}^{-1}+n_{2} n_{1}^{-1}\left(1-\Xi_{2} \Delta_{2}^{-1}\right)\right) /\left(\Xi_{2} \Delta_{2}^{-1}+n_{1} n_{2}^{-1}\left(1-\Xi_{1} \Delta_{1}^{-1}\right)\right)$. We distinguish two cases:

Case I: $\frac{\Xi_{2} \Delta_{2}^{-1}+\Xi_{1} \Delta_{1}^{-1}-1}{\left(1-\Xi_{1} \Delta_{1}^{-1}\right) n_{1} n_{2}^{-1}+\Xi_{2} \Delta_{2}^{-1}} \leq \frac{\Xi_{1} \Delta_{1}^{-1}+n_{2} n_{1}^{-1}\left(1-\Xi_{2} \Delta_{2}^{-1}\right)}{n_{1}\left(\Xi_{1} \Delta_{1}^{-1}+n_{2} n_{1}^{-1}\left(1-\Xi_{2} \Delta_{2}^{-1}\right)\right)+\left(n_{2}-1\right)\left(\left(1-\Xi_{1} \Delta_{1}^{-1}\right) n_{1} n_{2}^{-1}+\Xi_{2} \Delta_{2}^{-1}\right)}$. Using the expressions of $\Xi_{i}$ and $\Delta_{i}$, we get that the previous inequality is equivalent to (34). Moreover, after some algebra, we have that $q_{D}(\widehat{z}) \leq 0$. Consequently, $\widehat{z} \leq \bar{z}_{D}<z$, which implies that in this case $d_{1}+\lambda_{1}<d_{2}+\lambda_{2}$ holds. Note that inequality holds for $\rho$ close to 1 .

Case II: $\frac{\Xi_{2} \Delta_{2}^{-1}+\Xi_{1} \Delta_{1}^{-1}-1}{\left(1-\Xi_{1} \Delta_{1}^{-1}\right) n_{1} n_{2}^{-1}+\Xi_{2} \Delta_{2}^{-1}}>\frac{\Xi_{1} \Delta_{1}^{-1}+n_{2} n_{1}^{-1}\left(1-\Xi_{2} \Delta_{2}^{-1}\right)}{n_{1}\left(\Xi_{1} \Delta_{1}^{-1}+n_{2} n_{1}^{-1}\left(1-\Xi_{2} \Delta_{2}^{-1}\right)\right)+\left(n_{2}-1\right)\left(\left(1-\Xi_{1} \Delta_{1}^{-1}\right) n_{1} n_{2}^{-1}+\Xi_{2} \Delta_{2}^{-1}\right)}$. In this case, taking into account that $z$ is the unique positive solution of (20), (35) is equivalent to

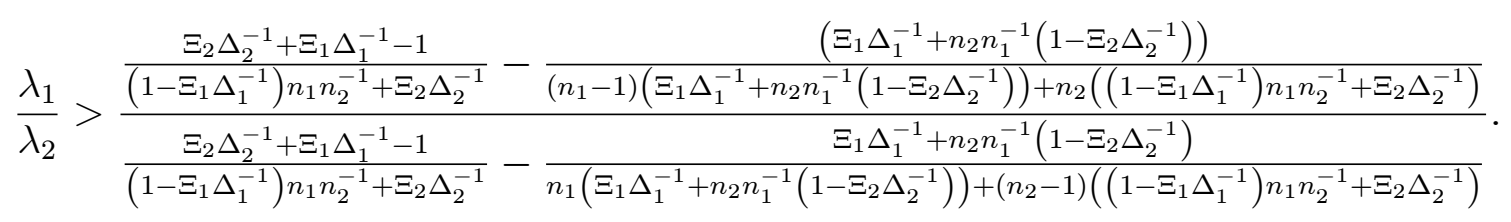

Taking into account that $\sigma_{\varepsilon_{1}}^{2} \geq \sigma_{\varepsilon_{2}}^{2}, \lambda_{1} \geq \lambda_{2}$, and $n_{1} \geq n_{2}$, and after some algebra, we get that both sides of the inequality are higher than (or equal to) 1 . Therefore, if the value of $\lambda_{1} / \lambda_{2}$ is high enough, then we obtain $d_{1}+\lambda_{1}>d_{2}+\lambda_{2}$. Otherwise, the opposite inequality holds. In short, the result indicates that the inequality $d_{1}+\lambda_{1}>d_{2}+\lambda_{2}$ holds whenever (a) the differences between groups stem mainly from transaction costs; ${ }^{36}$ and (b) $\lambda_{1} / \lambda_{2}$ is high enough. Finally, under complete information, the inequality (36) becomes $\frac{\lambda_{1}}{\lambda_{2}}>1$ and, therefore, we have that in this case $d_{1}+\lambda_{1}>d_{2}+\lambda_{2}$ iff $\lambda_{1}>\lambda_{2}$.

Proof of Proposition 4: (i) First, suppose that $\bar{\theta}_{1}=\bar{\theta}_{2}$. Then $\mathbb{E}[p]=\bar{\theta}_{1}-Q /\left(\frac{n_{1}}{d_{1}+\lambda_{1}}+\frac{n_{2}}{d_{2}+\lambda_{2}}\right)$. From Proposition 3 we know that $d_{i}$ and $d_{j}$ decrease with $n_{i}$, and increase with $\sigma_{\varepsilon_{i}}^{2}, \lambda_{i}$, and $\rho$. Using these results in the previous expression, we conclude that the expected price is increasing in $n_{i}$ and decreasing in $\lambda_{i}, \sigma_{\varepsilon_{i}}^{2}$, and $\rho$.

Now, suppose that $\bar{\theta}_{1} \neq \bar{\theta}_{2}$. The results we have just derived may not hold if $\left|\bar{\theta}_{1}-\bar{\theta}_{2}\right|$ is large enough. For example, let us focus on the relationship between the expected price and

\footnotetext{
${ }^{36}$ This claim follows because if $n_{1}=n_{2}$ and $\widehat{\sigma}_{\varepsilon_{1}}^{2}=\widehat{\sigma}_{\varepsilon_{2}}^{2}$, then the inequality given in (34) does not hold.
} 
$n_{1}$. To study this relationship, we first show that $n_{2}\left(d_{1}+\lambda_{1}\right) /\left(n_{1}\left(d_{2}+\lambda_{2}\right)\right)$ decreases with $n_{1}$. Recall that $d_{2}=\left(\left(n_{2}-1\right) n_{1} / \Omega_{2}+n_{1} n_{2} / \Omega_{1}\right)^{-1}$. Using the expressions of $\Omega_{i}, i=1,2$, we have

$$
1=\left(\frac{n_{2}-1}{\Upsilon_{2}+\left(\Upsilon_{2}-1\right) \frac{n_{2}\left(d_{1}+\lambda_{1}\right)}{n_{1}\left(d_{2}+\lambda_{2}\right)}}+\frac{n_{2}}{\Upsilon_{1} \frac{n_{2}\left(d_{1}+\lambda_{1}\right)}{n_{1}\left(d_{2}+\lambda_{2}\right)}+\Upsilon_{1}-1}\right)^{-1}+\frac{\lambda_{2}}{d_{2}+\lambda_{2}} .
$$

The fact that $d_{2}$ decreases with $n_{1}$ implies that $\lambda_{2} /\left(d_{2}+\lambda_{2}\right)$ increases with $n_{1}$. Then, the previous inequality tells us that $\frac{n_{2}-1}{\Upsilon_{2}+\left(\Upsilon_{2}-1\right) \frac{n_{2}\left(d_{1}+\lambda_{1}\right)}{n_{1}\left(d_{2}+\lambda_{2}\right)}}+\frac{n_{2}}{\Upsilon_{1} \frac{n_{2}\left(d_{1}+\lambda_{1}\right)}{n_{1}\left(d_{2}+\lambda_{2}\right)}+\Upsilon_{1}-1}$ increases with $n_{1}$. For this to be possible, $\frac{n_{2}\left(d_{1}+\lambda_{1}\right)}{n_{1}\left(d_{2}+\lambda_{2}\right)}$ needs to be decreasing in $n_{1}$. Given that the expected price satisfies $\mathbb{E}[p]=\left(1+\frac{n_{2}\left(d_{1}+\lambda_{1}\right)}{n_{1}\left(d_{2}+\lambda_{2}\right)}\right)^{-1} \bar{\theta}_{1}+\left(1-\left(1+\frac{n_{2}\left(d_{1}+\lambda_{1}\right)}{n_{1}\left(d_{2}+\lambda_{2}\right)}\right)^{-1}\right) \bar{\theta}_{2}-\left(\frac{n_{1}}{d_{1}+\lambda_{1}}+\frac{n_{2}}{d_{2}+\lambda_{2}}\right)^{-1} Q$, we have that the relationship between the expected price and $n_{1}$ is ambiguous. For instance, if $\bar{\theta}_{2}$ is low enough, then the fact that $d_{1}, d_{2}$, and $n_{2}\left(d_{1}+\lambda_{1}\right) /\left(n_{1}\left(d_{2}+\lambda_{2}\right)\right)$ are decreasing in $n_{1}$ allows us to conclude that the expected price increases with $n_{1}$. However, if $\bar{\theta}_{2}$ is large and $\bar{\theta}_{1}$ and $Q$ are low enough, then the expected price decreases with $n_{1}$.

(ii) From the expression for the expected revenue it follows that $Q E[p]$ increases with $\bar{\theta}_{i}, i=1,2$. In addition, direct computation yields that $Q=\left(\frac{n_{1}}{d_{1}+\lambda_{1}} \bar{\theta}_{1}+\frac{n_{2}}{d_{2}+\lambda_{2}} \bar{\theta}_{2}\right) / 2$. Using Proposition 3 , it follows that this quantity increases with $n_{i}$ and $\bar{\theta}_{i}$ and decreases with $\rho, \lambda_{i}$ and $\sigma_{\varepsilon_{i}}^{2}, i=1,2$.

Proof of Corollary 3: Using the expression for the expected price, it follows that

$$
\left(\min \left\{\bar{\theta}_{1}, \bar{\theta}_{2}\right\}-\frac{Q}{\frac{n_{1}}{d_{1}+\lambda_{1}}+\frac{n_{2}}{d_{2}+\lambda_{2}}}\right) Q \leq \mathbb{E}[p] Q \leq\left(\max \left\{\bar{\theta}_{1}, \bar{\theta}_{2}\right\}-\frac{Q}{\frac{n_{1}}{d_{1}+\lambda_{1}}+\frac{n_{2}}{d_{2}+\lambda_{2}}}\right) Q .
$$

Notice both the left-hand side $(L H S)$ and the right-hand side $(R H S)$ of this expression correspond to the expected revenue in an auction where all participants have an expected valuation of $\min \left\{\bar{\theta}_{1}, \bar{\theta}_{2}\right\}$ and of $\max \left\{\bar{\theta}_{1}, \bar{\theta}_{2}\right\}$, respectively. Using Proposition 4(i), we know that both $L H S$ and $R H S$ increase with $n_{i}$ but decrease with $\lambda_{i}$ and $\sigma_{\varepsilon_{i}}^{2}$. Hence, we obtain that $Q E[p]$ is lower than the expected revenue of the symmetric auction in which both groups are ex-ante identical, with large size (each group with $\max \left\{n_{1}, n_{2}\right\}$ bidders), with high expected valuation $\left(\max \left\{\bar{\theta}_{1}, \bar{\theta}_{2}\right\}\right)$, low transaction $\operatorname{costs}\left(\min \left\{\lambda_{1}, \lambda_{2}\right\}\right)$, and precise signals $\left(\min \left\{\sigma_{\varepsilon_{1}}^{2}, \sigma_{\varepsilon_{2}}^{2}\right\}\right)$, and larger than the expected revenue of the symmetric auction in which both groups are ex-ante identical but with the opposite characteristics (i.e., $\min \left\{n_{1}, n_{2}\right\}, \min \left\{\bar{\theta}_{1}, \bar{\theta}_{2}\right\}, \max \left\{\lambda_{1}, \lambda_{2}\right\}$, and $\left.\max \left\{\sigma_{\varepsilon_{1}}^{2}, \sigma_{\varepsilon_{2}}^{2}\right\}\right)$.

Lemma A3. The equilibrium quantities solve the following distorted benefit maximization program:

$$
\max _{x_{1}, x_{2}} \mathbb{E}\left[n_{1}\left(\theta_{1} x_{1}-\left(d_{1}+\lambda_{1}\right) x_{1}^{2} / 2\right)+n_{2}\left(\theta_{2} x_{2}-\left(d_{2}+\lambda_{2}\right) x_{2}^{2} / 2\right) \mid t\right]
$$




$$
\text { s.t. } n_{1} x_{1}+n_{2} x_{2}=Q \text {, }
$$

taking as given the equilibrium parameters $d_{1}$ and $d_{2}$.

Proof: The Lagrangian function of the maximization program is given by

$$
\mathcal{L}\left(x_{1}, x_{2}, \mu\right)=n_{1}\left(t_{1} x_{1}-\left(d_{1}+\lambda_{1}\right) x_{1}^{2} / 2\right)+n_{2}\left(t_{2} x_{2}-\left(d_{2}+\lambda_{2}\right) x_{2}^{2} / 2\right)-\mu\left(n_{1} x_{1}+n_{2} x_{2}-Q\right),
$$

where $\mu$ denotes the Lagrange multiplier. Differentiating, we obtain the following FOCs:

$$
\begin{aligned}
n_{1}\left(t_{1}-\left(d_{1}+\lambda_{1}\right) x_{1}\right)-\mu n_{1} & =0, \\
n_{2}\left(t_{2}-\left(d_{2}+\lambda_{2}\right) x_{2}\right)-\mu n_{2} & =0, \text { and } \\
n_{1} x_{1}+n_{2} x_{2} & =Q .
\end{aligned}
$$

From (37) and (38), it follows that $x_{i}=\left(t_{i}-\mu\right) /\left(d_{i}+\lambda_{i}\right), i=1,2$. Substituting these expressions in (39) and operating, we have $\mu=\left(\frac{n_{1} t_{1}}{d_{1}+\lambda_{1}}+\frac{n_{2} t_{2}}{d_{2}+\lambda_{2}}-Q\right)\left(\frac{n_{1}}{d_{1}+\lambda_{1}}+\frac{n_{2}}{d_{2}+\lambda_{2}}\right)^{-1}$. Then, plugging this expression into (37) and (38), we get the expressions of the equilibrium quantities given in (4). In addition, since the objective function is concave and the constraint is a linear equation, we conclude that the critical point is a global maximum. Hence, the equilibrium quantities are the solutions of the optimization problem stated in Lemma A3.

Proof of Proposition 5: In the competitive setup, the FOC of the two optimization problems are given by $\mathbb{E}\left[\theta_{i} \mid s_{i}, p\right]-p-\lambda_{i} x_{i}=0, i=1,2$. Doing similar computations as in the proof of Lemma A1, we derive the following system of equations: ${ }^{37}$

$$
\begin{aligned}
b_{i} & =\left(\left(1-\Xi_{i}\right) \bar{\theta}_{i}-\Psi_{i} \bar{\theta}_{j}+\Psi_{i}\left(\frac{q-\mu_{i} b_{i}-\mu_{j} b_{j}}{\mu_{j} a_{j}}\right)\right) / \lambda_{i}, \\
a_{i} & =\left(\Xi_{i}-\frac{\mu_{i}}{\mu_{j}} \frac{a_{i}}{a_{j}} \Psi_{i}\right) / \lambda_{i}, \text { and } \\
c_{i} & =\left(1-\Psi_{i}\left(\frac{\mu_{i} c_{i}+\mu_{j} c_{j}}{\mu_{j} a_{j}}\right)\right) / \lambda_{i}, i, j=1,2, j \neq i .
\end{aligned}
$$

Note that $a_{i} / a_{j}=\left(\left(\Xi_{i}-\frac{\mu_{i}}{\mu_{j}} \frac{a_{i}}{a_{j}} \Psi_{i}\right) / \lambda_{i}\right) /\left(\left(\Xi_{j}-\frac{\mu_{j}}{\mu_{i}} \frac{a_{j}}{a_{i}} \Psi_{j}\right) / \lambda_{j}\right)$. Hence,

$$
a_{i} / a_{j}=\mu_{j}\left(\Psi_{j} \lambda_{i} \mu_{j}+\Xi_{i} \lambda_{j} \mu_{i}\right) /\left(\mu_{i}\left(\Psi_{i} \lambda_{j} \mu_{i}+\lambda_{i} \Xi_{j} \mu_{j}\right)\right) \text {. }
$$

Then, plugging the previous expression into (41), we get

$$
a_{i}=\frac{\mu_{j}\left(\Xi_{i} \Xi_{j}-\Psi_{i} \Psi_{j}\right)}{\mu_{j} \Xi_{j} \lambda_{i}+\mu_{i} \Psi_{i} \lambda_{j}} .
$$

\footnotetext{
${ }^{37}$ To ease the notation the superscript $o$ is omitted in this proof.
} 
Furthermore, using (40), and after some algebra, we have

$$
\mu_{i} b_{i}+\mu_{j} b_{j}=\frac{\frac{\mu_{i}}{\lambda_{i}}\left(\left(1-\Xi_{i}\right) \bar{\theta}_{i}-\Psi_{i} \bar{\theta}_{j}+\frac{\Psi_{i}}{\mu_{j} a_{j}} q\right)+\frac{\mu_{j}}{\lambda_{j}}\left(\left(1-\Xi_{j}\right) \bar{\theta}_{j}-\Psi_{j} \bar{\theta}_{i}+\frac{\Psi_{j}}{\mu_{i} a_{i}} q\right)}{\frac{\Psi_{i}}{\lambda_{i}} \frac{\mu_{i}}{\mu_{j} a_{j}}+\frac{\Psi_{j}}{\lambda_{j}} \frac{\mu_{j}}{\mu_{i} a_{i}}+1} .
$$

Substituting (43) and the last expression into (40), it follows that

$$
b_{i}=a_{i}\left(\frac{\Xi_{j} \bar{\theta}_{i}-\Psi_{i} \bar{\theta}_{j}}{\Xi_{i} \Xi_{j}-\Psi_{i} \Psi_{j}}-\bar{\theta}_{i}\right)+\frac{\lambda_{j} \Psi_{i}}{\mu_{j} \Xi_{j} \lambda_{i}+\mu_{i} \Psi_{i} \lambda_{j}} q
$$

In addition, from (42), and after some algebra, we get

$$
\mu_{i} c_{i}+c_{j} \mu_{j}=\left(\frac{\mu_{i}}{\lambda_{i}}+\frac{\mu_{j}}{\lambda_{j}}\right) /\left(\frac{\Psi_{i}}{\lambda_{i}} \frac{\mu_{i}}{\mu_{j} a_{j}}+\frac{\Psi_{j}}{\lambda_{j}} \frac{\mu_{j}}{\mu_{i} a_{i}}+1\right)
$$

Using (43) and the last expression in (42), we have

$$
c_{i}=\frac{\mu_{j}\left(\Xi_{j}-\Psi_{i}\right)}{\mu_{j} \Xi_{j} \lambda_{i}+\mu_{i} \Psi_{i} \lambda_{j}} .
$$

Finally, taking into account the expressions for $\Xi_{i}, \Xi_{j}, \Psi_{i}$, and $\Psi_{j}$, we obtain the expressions stated in Proposition 5.

Proof of Proposition 6: Performing similar computations as in the proof of Lemma A1, we obtain that the equilibrium coefficients with subsidies $\kappa_{i}=d_{i}\left(c_{1}^{o}, c_{2}^{o}\right)$ satisfy

$$
\begin{aligned}
b_{i} & =\frac{\left(1-\Xi_{i}\right) \bar{\theta}_{i}-\Psi_{i} \bar{\theta}_{j}-\frac{\Psi_{i}\left(n_{i} b_{i}+n_{j} b_{j}-Q\right)}{n_{j} a_{j}}}{d_{i}+\lambda_{i}-d_{i}\left(c_{1}^{o}, c_{2}^{o}\right)}, a_{i}=\frac{\Xi_{i}-\frac{n_{i} a_{i}}{n_{j} a_{j}} \Psi_{i}}{d_{i}+\lambda_{i}-d_{i}\left(c_{1}^{o}, c_{2}^{o}\right)}>0, \text { and } \\
c_{i} & =\frac{1-\frac{\Psi_{i}\left(n_{i} c_{i}+n_{j} c_{j}\right)}{n_{j} a_{j}}}{d_{i}+\lambda_{i}-d_{i}\left(c_{1}^{o}, c_{2}^{o}\right)}, i, j=1,2, j \neq i .
\end{aligned}
$$

Comparing this system of equations and the one derived in the proof of Proposition 5, using $Q=\left(n_{1}+n_{2}\right) q$ and $\mu_{i}=n_{i} /\left(n_{1}+n_{2}\right)$ for $i=1,2$, we obtain that the equilibrium coefficients of the price-taking equilibrium solves this system. Therefore, we conclude that the quadratic subsidies $\kappa_{i} x_{i}^{2} / 2$, with $\kappa_{i}=d_{i}\left(c_{1}^{o}, c_{2}^{o}\right), i=1,2$, induce an efficient allocation.

Lemma A4. The expected deadweight loss at an anonymous allocation $\left(x_{1}(t), x_{2}(t)\right)$ satisfies

$$
\mathbb{E}[D W L]=\frac{1}{2} \lambda_{1} n_{1} \mathbb{E}\left[\left(x_{1}(t)-x_{1}^{o}(t)\right)^{2}\right]+\frac{1}{2} \lambda_{2} n_{2} \mathbb{E}\left[\left(x_{2}(t)-x_{2}^{o}(t)\right)^{2}\right] .
$$

Proof: Notice that $E T S=\mathbb{E}[\mathbb{E}[T S \mid t]]$, where

$$
\begin{aligned}
\mathbb{E}[T S \mid t]= & \mathbb{E}\left[n_{1}\left(\theta_{1} x_{1}(t)-\lambda_{1}\left(x_{1}(t)\right)^{2} / 2\right)+n_{2}\left(\theta_{2} x_{2}(t)-\lambda_{2}\left(x_{2}(t)\right)^{2} / 2\right) \mid t\right]= \\
& n_{1}\left(t_{1} x_{1}(t)-\lambda_{1}\left(x_{1}(t)\right)^{2} / 2\right)+n_{2}\left(t_{2} x_{2}(t)-\lambda_{2}\left(x_{2}(t)\right)^{2} / 2\right) .
\end{aligned}
$$


A Taylor series expansion of $\mathbb{E}[T S \mid t]$ around the price-taking equilibrium $\left(x_{1}^{o}(t), x_{2}^{o}(t)\right)$, stopping at the second term due to the fact that $\mathbb{E}[T S \mid t]$ is quadratic, yields

$$
\begin{aligned}
\mathbb{E}[T S \mid t](x(t))= & \mathbb{E}[T S \mid t]\left(x^{o}(t)\right)+\nabla \mathbb{E}[T S \mid t]\left(x^{o}(t)\right)\left(x(t)-x^{o}(t)\right)+ \\
& +\frac{1}{2}\left(x(t)-x^{o}(t)\right)^{\prime} D^{2} \mathbb{E}[T S \mid t]\left(x^{o}(t)\right)\left(x(t)-x^{o}(t)\right),
\end{aligned}
$$

where $\nabla \mathbb{E}[T S \mid t]\left(x^{o}(t)\right)$ and $D^{2} \mathbb{E}[T S \mid t]\left(x^{o}(t)\right)$ are, respectively, the gradient and the Hessian matrix of $\mathbb{E}[T S \mid t]$ evaluated at $x^{o}(t)$. By optimality,

$$
\nabla \mathbb{E}[T S \mid t]\left(x^{o}(t)\right)=(0,0) .
$$

In addition, $D^{2} \mathbb{E}[T S \mid t]\left(x^{o}(t)\right)=\left(\begin{array}{cc}-\lambda_{1} n_{1} & 0 \\ 0 & -\lambda_{2} n_{2}\end{array}\right)$. Hence,

$$
\mathbb{E}[T S \mid t](x(t))-\mathbb{E}[T S \mid t]\left(x^{o}(t)\right)=-\frac{1}{2} \lambda_{1} n_{1}\left(x_{1}(t)-x_{1}^{o}(t)\right)^{2}-\frac{1}{2} \lambda_{2} n_{2}\left(x_{2}(t)-x_{2}^{o}(t)\right)^{2}
$$

and, therefore, (46) is satisfied.

Proof of Proposition \%: (i) Suppose that $Q=0$. Then, $\mathbb{E}[D W L]$ is given by

$$
\mathbb{E}[D W L]=\frac{n_{1} n_{2}\left(n_{2} d_{1}+n_{1} d_{2}\right)^{2}}{2\left(n_{2} \lambda_{1}+n_{1} \lambda_{2}\right) u^{2}} \mathbb{E}\left(t_{1}-t_{2}\right)^{2} .
$$

Hence,

$$
\frac{d \mathbb{E}[D W L]}{d \sigma_{\varepsilon_{1}}^{2}}=\frac{\partial \mathbb{E}[D W L]}{\partial d_{1}} \frac{\partial d_{1}}{\partial \sigma_{\varepsilon_{1}}^{2}}+\frac{\partial \mathbb{E}[D W L]}{\partial d_{2}} \frac{\partial d_{2}}{\partial \sigma_{\varepsilon_{1}}^{2}}+\frac{\partial \mathbb{E}[D W L]}{\partial \sigma_{\varepsilon_{1}}^{2}} .
$$

It is easy to see that in this case $\partial \mathbb{E}[D W L] / \partial d_{i}>0, i=1,2$, and $\partial \mathbb{E}[D W L] / \partial \sigma_{\varepsilon_{1}}^{2}<0$. Combining these results with Proposition 3, we have that the first two terms of $d \mathbb{E}[D W L] / d \sigma_{\varepsilon_{1}}^{2}$ are positive, while the last one is negative.

We know that $d_{1}$ and $d_{2}$ are independent of $\sigma_{\varepsilon_{1}}^{2}$ when $\rho=0$. By continuity, we know that for very low values of $\rho$ is $\partial d_{1} / \partial \sigma_{\varepsilon_{1}}^{2}$ and $\partial d_{2} / \partial \sigma_{\varepsilon_{1}}^{2}$ are positive, but very low. Hence, we conclude that the last term in $d \mathbb{E}[D W L] / d \sigma_{\varepsilon_{1}}^{2}$ dominates and, hence, in this case we have that $d \mathbb{E}[D W L] / d \sigma_{\varepsilon_{1}}^{2}<0$ although $\partial d_{1} / \partial \sigma_{\varepsilon_{1}}^{2}>0, i=1,2$. By contrast, if we consider the case in which $\rho$ is not low and $\left(\bar{\theta}_{1}-\bar{\theta}_{2}\right)^{2}$ is high enough, then the first two terms in $d \mathbb{E}[D W L] / d \sigma_{\varepsilon_{1}}^{2}$ dominate, which implies that $d \mathbb{E}[D W L] / d \sigma_{\varepsilon_{1}}^{2}>0, i=1,2$.

(ii) Omitted since it is trivial.

(iii) When groups are symmetric $\left(n_{2}=n_{1}=n, \lambda_{1}=\lambda_{2}=\lambda\right.$, and $\left.\sigma_{\varepsilon_{1}}^{2}=\sigma_{\varepsilon_{2}}^{2}=\sigma_{\varepsilon}^{2}\right)$ $d_{1}=d_{2}=d$ and $\lambda_{2} d_{1}-\lambda_{1} d_{2}=0$. Therefore, the expected deadweight loss consists of only one term, the first one, that is independent of $Q$. Moreover, we obtain that $\mathbb{E}[D W L \mid t]=$ $n d^{2}\left(t_{2}-t_{1}\right)^{2} /\left(4(d+\lambda)^{2} \lambda\right)$ and since $\partial \mathbb{E}[D W L \mid t] / \partial d>0$, we conclude that an increase in an information parameter $\left(\rho\right.$ or $\widehat{\sigma}_{\varepsilon}^{2}$ ) raises both $d$ and $\mathbb{E}[D W L \mid t]$. 
However, with asymmetric groups the previous results may not hold. In this case, suppose that $Q$ is large enough. Then, for $i=1,2, j \neq i$,

$$
\operatorname{sgn}\left\{\frac{\partial E[D W L \mid t]}{\partial d_{i}}\right\}=\operatorname{sgn}\left\{\partial\left(\frac{n_{1} n_{2}\left(\lambda_{1} d_{2}-\lambda_{2} d_{1}\right)^{2}}{2\left(\lambda_{1} n_{2}+\lambda_{2} n_{1}\right) u^{2}} Q^{2}\right) / \partial d_{i}\right\}=\operatorname{sgn}\left\{\lambda_{j} d_{i}-\lambda_{i} d_{j}\right\} .
$$

When $Q$ is large enough we have that if $d_{i} / d_{j}>\lambda_{i} / \lambda_{j}$, then $\partial E[D W L \mid t] / \partial d_{i}>0$ and $\partial E[D W L \mid t] / \partial d_{j}<0$. Thus, with asymmetric groups price impact $\left(d_{1}, d_{2}\right)$ and the $E[D W L \mid t]$ are not always positively associated, given predicted values $t$, for changes in information parameters.

Proof of Proposition 8: Using (23) and (24), it follows that $\lim _{n_{1} \rightarrow \infty} \bar{z}_{N}=\lim _{n_{1} \rightarrow \infty} \bar{z}_{D}=0$. Furthermore, after some algebra, we have that the necessary and sufficient condition for the existence of an equilibrium (i.e., $\lim _{n_{1} \rightarrow \infty} \bar{z}_{N} / \bar{z}_{D}>1$ ) is equivalent to $n_{2}>\bar{n}_{2}\left(\rho, \widehat{\sigma}_{\varepsilon_{1}}^{2}, \widehat{\sigma}_{\varepsilon_{2}}^{2}\right)$, where

$$
\bar{n}_{2}\left(\rho, \widehat{\sigma}_{\varepsilon_{1}}^{2}, \widehat{\sigma}_{\varepsilon_{2}}^{2}\right)=\frac{\rho\left((2-\rho) \widehat{\sigma}_{\varepsilon_{2}}^{2}+2\left(1-\rho^{2}\right)\right) \widehat{\sigma}_{\varepsilon_{1}}^{2}}{\left(1-\rho^{2}\right)\left(\left(1+\widehat{\sigma}_{\varepsilon_{1}}^{2}\right)\left(1+\widehat{\sigma}_{\varepsilon_{2}}^{2}\right)-\rho^{2}\right)} .
$$

Moreover, taking the limit in (20), it follows that $\lim _{n_{1} \rightarrow \infty} z=0$ and

$$
\lim _{n_{1} \rightarrow \infty} n_{1} z=n_{2} \Xi_{1} \Delta_{1}^{-1} /\left(1-\Xi_{1} \Delta_{1}^{-1}\right) .
$$

Using the expressions included in the statement of Lemma A2, and after some tedious algebra, we get $\lim _{n_{1} \rightarrow \infty} b_{1}=q, \lim _{n_{1} \rightarrow \infty} a_{1}=0, \lim _{n_{1} \rightarrow \infty} c_{1}=0, \lim _{n_{1} \rightarrow \infty} a_{2}=\Delta_{2} \lim _{n_{1} \rightarrow \infty} c_{2}$,

$$
\begin{aligned}
\lim _{n_{1} \rightarrow \infty} b_{2}= & \frac{\widehat{\sigma}_{\varepsilon_{2}}^{2}\left(\frac{\left(n_{2}-1\right)\left(1-\rho^{2}\right)}{\left(1-\rho^{2}+\widehat{\sigma}_{\varepsilon_{2}}^{2}\right)}+\frac{\left(1-\rho^{2}+\widehat{\sigma}_{\varepsilon_{1}}^{2}(1-2 \rho)\right)}{\left(1+\widehat{\sigma}_{\varepsilon_{1}}^{2}\right)\left(1+\widehat{\sigma}_{\varepsilon_{2}}^{2}\right)-\rho^{2}}\right)\left(\bar{\theta}_{2}-\rho \bar{\theta}_{1}+q \rho \lambda_{1}\right)}{(1-\rho) \lambda_{2}\left(n_{2}(1+\rho)-\frac{\rho \widehat{\sigma}_{\varepsilon_{1}}^{2}\left(1+\rho+\widehat{\sigma}_{\varepsilon_{2}}^{2}\right)}{\left(1+\widehat{\sigma}_{\varepsilon_{1}}^{2}\right)\left(1+\widehat{\sigma}_{\varepsilon_{2}}^{2}\right)-\rho^{2}}\right)}+ \\
& +q \frac{\rho^{2} \widehat{\sigma}_{\varepsilon_{2}}^{2} \widehat{\sigma}_{\varepsilon_{1}}^{2}}{\lim _{2}\left(1-\rho^{2}\right)\left(\left(1+\widehat{\sigma}_{\varepsilon_{1}}^{2}\right)\left(1+\widehat{\sigma}_{\varepsilon_{2}}^{2}\right)-\rho^{2}\right)}, \text { and } \\
\lim _{1 \rightarrow \infty}= & \frac{n_{2}-\bar{n}_{2}\left(\rho, \widehat{\sigma}_{\varepsilon_{1}}^{2}, \widehat{\sigma}_{\varepsilon_{2}}^{2}\right)}{\lambda_{2} \frac{1-\rho^{2}+\widehat{\sigma}_{\varepsilon_{2}}^{2}}{1-\rho}\left(\frac{n_{2}}{\left(1+\rho+\widehat{\sigma}_{\varepsilon_{2}}^{2}\right)}-\frac{\rho \widehat{\sigma}_{\varepsilon_{1}}^{2}}{(1+\rho)\left(\left(1+\widehat{\sigma}_{\varepsilon_{1}}^{2}\right)\left(1+\widehat{\sigma}_{\varepsilon_{2}}^{2}\right)-\rho^{2}\right)}\right)}
\end{aligned}
$$

Next, in relation to the expressions for $d_{1}$ and $d_{2}$, we have that

$$
\lim _{n_{1} \rightarrow \infty} d_{1}=\lim _{n_{1} \rightarrow \infty}\left(\left(n_{1}-1\right) c_{1}+n_{2} c_{2}\right)^{-1}=\left(\lim _{n_{1} \rightarrow \infty}\left(\frac{\left(n_{1}-1\right)}{n_{1}} n_{1} z+n_{2}\right) \lim _{n_{1} \rightarrow \infty} c_{2}\right)^{-1}>0 .
$$

The fact that $n_{1} z$ and $c_{2}$ converge to a positive finite number (see (47) and (48)) implies that $d_{1}$ does not converge to zero (provided that $\rho \widehat{\sigma}_{\varepsilon_{1}}^{2}>0$; if $\rho \widehat{\sigma}_{\varepsilon_{1}}^{2}=0$, then it is easy to see that 
$\left.\lim _{n_{1} \rightarrow \infty} n_{1} z=\infty\right)$. A similar result is obtained with the limit of $d_{2}$. In particular,

$$
\lim _{n_{1} \rightarrow \infty} d_{2}=\left(\left(\lim _{n_{1} \rightarrow \infty} n_{1} z+n_{2}-1\right) \lim _{n_{1} \rightarrow \infty} c_{2}\right)^{-1}>\lim _{n_{1} \rightarrow \infty} d_{1}>0
$$

Proof of Proposition 9: Suppose that $n_{1}$ and $n_{2}$ go to infinity and that $n_{1} /\left(n_{1}+n_{2}\right)$ converges to $\mu_{1}$. Taking limits in the equation that characterizes $z$ (i.e., Equation (20)) and operating, we have

$$
z=\frac{\Xi_{1} \Delta_{1}^{-1}+\mu_{2} \mu_{1}^{-1}\left(1-\Xi_{2} \Delta_{2}^{-1}\right) \lambda_{1} \lambda_{2}^{-1}}{\mu_{1} \mu_{2}^{-1}\left(1-\Xi_{1} \Delta_{1}^{-1}\right)+\Xi_{2} \Delta_{2}^{-1} \lambda_{1} \lambda_{2}^{-1}}
$$

Moreover, taking the limit in the expressions of the equilibrium coefficients given in Lemma A2, it follows that

$$
\begin{aligned}
b_{i} & =a_{i}\left(\frac{\Xi_{j} \bar{\theta}_{i}-\Psi_{i} \bar{\theta}_{j}}{\Xi_{i} \Xi_{j}-\Psi_{i} \Psi_{j}}-\bar{\theta}_{i}\right)+\frac{\Psi_{i}}{\mu_{i} \mu_{j}} \frac{\mu_{i} \Xi_{j} \frac{a_{i}}{a_{j}}-\mu_{j} \Psi_{j}}{\left(\Xi_{i} \Xi_{j}-\Psi_{i} \Psi_{j}\right)} q, a_{i}=\Delta_{i} c_{i}, i, j=1,2, j \neq i, \\
c_{1} & =\frac{\Xi_{1} \Delta_{1}^{-1}-\mu_{1} \mu_{2}^{-1}\left(1-\Xi_{1} \Delta_{1}^{-1}\right) z}{\lambda_{1}}, \text { and } c_{2}=\frac{\Xi_{2} \Delta_{2}^{-1}-\mu_{2} \mu_{1}^{-1}\left(1-\Xi_{2} \Delta_{2}^{-1}\right) z^{-1}}{\lambda_{2}} .
\end{aligned}
$$

Substituting (49) in the previous expressions and after some algebra, we get

$$
\begin{aligned}
b_{i} & =a_{i}\left(\frac{\Xi_{j} \bar{\theta}_{i}-\Psi_{i} \bar{\theta}_{j}}{\Xi_{i} \Xi_{j}-\Psi_{i} \Psi_{j}}-\bar{\theta}_{i}\right)+\frac{\lambda_{j} \Psi_{i}}{\mu_{j} \Xi_{j} \lambda_{i}+\mu_{i} \Psi_{i} \lambda_{j}} q, \\
a_{i} & =\frac{\mu_{j}\left(\Xi_{i} \Xi_{j}-\Psi_{i} \Psi_{j}\right)}{\mu_{j} \Xi_{j} \lambda_{i}+\mu_{i} \Psi_{i} \lambda_{j}}, \text { and } \\
c_{i} & =\frac{\mu_{j}\left(\Xi_{j}-\Psi_{i}\right)}{\mu_{j} \Xi_{j} \lambda_{i}+\mu_{i} \Psi_{i} \lambda_{j}}, i, j=1,2, j \neq i .
\end{aligned}
$$

Next, we derive the equilibrium in the following continuous setup: Consider now that there is a continuum of bidders $[0,1]$. Let $q$ denote the aggregate quantity supplied in the market. Suppose that a fraction $\mu_{1}$ of these bidders are traders of type 1 and the remainder fraction, $\mu_{2}$, are bidders of type 2 .

Consider a trader of type $i$. This bidder chooses to maximize

$$
\mathbb{E}\left[\pi_{i} \mid s_{i}, p\right]=\left(\mathbb{E}\left[\theta_{i} \mid s_{i}, p\right]-p\right) x_{i}-\lambda_{i} x_{i}^{2} / 2
$$

The FOC is given by $\mathbb{E}\left[\theta_{i} \mid s_{i}, p\right]-p-\lambda_{i} x_{i}=0$, or equivalently,

$$
X_{i}\left(s_{i}, p\right)=\left(\mathbb{E}\left[\theta_{i} \mid s_{i}, p\right]-p\right) / \lambda_{i}
$$

Positing linear strategies, the market clearing condition implies that

$$
p=\left(\mu_{i}\left(b_{i}+a_{i} s_{i}\right)+\mu_{j}\left(b_{j}+a_{j} s_{j}\right)-q\right) /\left(\mu_{i} c_{i}+\mu_{j} c_{j}\right),
$$


provided that $\mu_{i} c_{i}+\mu_{j} c_{j} \neq 0$. Using the expression for $p$ and assuming that $a_{i} \neq 0, i=1,2$, it follows that $\mathbb{E}\left[\theta_{i} \mid s_{i}, p\right]=\mathbb{E}\left[\theta_{i} \mid s_{i}, s_{j}\right]$. Hence, $\mathbb{E}\left[\theta_{i} \mid s_{i}, p\right]=\bar{\theta}_{i}+\Xi_{i}\left(s_{i}-\bar{\theta}_{i}\right)+\Psi_{i}\left(s_{j}-\bar{\theta}_{j}\right)$. Using (54), $s_{j}=\left(q-\mu_{i} b_{i}-\mu_{j} b_{j}-\mu_{i} a_{i} s_{i}+p\left(\mu_{i} c_{i}+\mu_{j} c_{j}\right)\right) /\left(\mu_{j} a_{j}\right)$. Therefore,

$$
\mathbb{E}\left[\theta_{i} \mid s_{i}, p\right]=\bar{\theta}_{i}+\Xi_{i}\left(s_{i}-\bar{\theta}_{i}\right)+\Psi_{i}\left(\left(q-\mu_{i} b_{i}-\mu_{j} b_{j}-\mu_{i} a_{i} s_{i}+p\left(\mu_{i} c_{i}+\mu_{j} c_{j}\right)\right) /\left(\mu_{j} a_{j}\right)-\bar{\theta}_{j}\right) .
$$

Substituting this expression in (53), and identifying coefficients, it follows that

$$
\begin{aligned}
b_{i} & =\left(\left(1-\Xi_{i}\right) \bar{\theta}_{i}-\Psi_{i} \bar{\theta}_{j}+\Psi_{i}\left(q-\left(\mu_{i} b_{i}+\mu_{j} b_{j}\right)\right) /\left(\mu_{j} a_{j}\right)\right) / \lambda_{i}, \\
a_{i} & =\left(\Xi_{i}-\Psi_{i} \mu_{i} a_{i} /\left(\mu_{j} a_{j}\right)\right) / \lambda_{i}, \text { and } \\
c_{i} & =\left(1-\Psi_{i}\left(\mu_{i} c_{i}+c_{j} \mu_{j}\right) /\left(\mu_{j} a_{j}\right)\right) / \lambda_{i}, i, j=1,2, j \neq i .
\end{aligned}
$$

We have that the previous system is identical to the system of equations given in (40)-(42), with solution given in (43), (44) and (45), which are identical to the expressions given in (50)-(52). We conclude that the equilibrium coefficients of the limiting case converge to the equilibrium coefficients of the continuous setup. Moreover, the equilibrium coefficients given in the statement of Proposition 5 coincide with the equilibrium coefficients in the continuous setup. 


\section{Appendix B}

\section{Bid shading and expected discount}

Recall that $\tilde{t}=\left(n_{1} t_{1}+n_{2} t_{2}\right) /\left(n_{1}+n_{2}\right)$. From the demand of bidders it follows that $p(t)=$ $t_{i}-\left(d_{i}+\lambda_{i}\right) x_{i}(t), i=1,2$. For a trader of type $i$, the expected marginal benefit of buying $x_{i}$ units of the asset is $t_{i}-\lambda_{i} x_{i}$. Hence, the average marginal benefit is given by $\tilde{t}-$ $\left(\lambda_{1} n_{1} x_{1}+\lambda_{2} n_{2} x_{2}\right) /\left(n_{1}+n_{2}\right)$. The magnitude of (average) bid shading is the difference between the average marginal valuation and the auction price, that is, $\left(d_{1} n_{1} x_{1}+d_{2} n_{2} x_{2}\right) /\left(n_{1}+n_{2}\right){ }^{38}$ We can use Equation (4) to write bid shading as

$$
\frac{\left(n_{2} d_{2}\left(d_{1}+\lambda_{1}\right)+n_{1} d_{1}\left(d_{2}+\lambda_{2}\right)\right) Q}{\left(n_{1}+n_{2}\right)\left(n_{1}\left(d_{2}+\lambda_{2}\right)+n_{2}\left(d_{1}+\lambda_{1}\right)\right)}+\frac{\left(t_{2}-t_{1}\right)\left(d_{2}-d_{1}\right) n_{2} n_{1}}{\left(n_{1}+n_{2}\right)\left(n_{1}\left(d_{2}+\lambda_{2}\right)+n_{2}\left(d_{1}+\lambda_{1}\right)\right)} .
$$

At this juncture, some additional remarks are in order.

- Bid shading increases with $Q$ and decreases when the group that values more the asset, say group $1,\left(t_{1}>t_{2}\right)$ has less price impact $\left(d_{1}<d_{2}\right)$.

- When $d_{1}=d_{2}=d$ as in the symmetric case, for instance, bid shading consists of only one term (the first one) and it is equal to $d Q /\left(n_{1}+n_{2}\right)$.

- When $d_{1} \neq d_{2}$, the second term of (55) is negative and bid shading decreases whenever the group that values the asset more highly $\left(t_{i}>t_{j}\right)$ has less price impact $\left(d_{i}<d_{j}\right)$.

- If group 1 has higher transaction costs $\left(\lambda_{1}>\lambda_{2}\right)$, is more numerous $\left(n_{1}>n_{2}\right)$, and is less informed $\left(\sigma_{\varepsilon_{1}}^{2}>\sigma_{\varepsilon_{2}}^{2}\right)$ than group 2 , then $c_{1}<c_{2}$, and so $d_{1}<d_{2}$. If $t_{1}>t_{2}$, then the second term of (55) is negative and the two terms have opposite signs. Therefore, if $Q$ is low (e.g., $Q=0$ ) or if the difference in predicted values of the asset is high, then negative bid shading obtains.

The expected discount is defined as $\mathbb{E}[\tilde{t}]-\mathbb{E}[p(t)]$. Using (6), some algebra yields the following expression for the expected discount:

$$
\frac{\left(d_{1}+\lambda_{1}\right)\left(d_{2}+\lambda_{2}\right)}{n_{1}\left(d_{2}+\lambda_{2}\right)+n_{2}\left(d_{1}+\lambda_{1}\right)} Q+\frac{n_{1} n_{2}\left(d_{2}+\lambda_{2}-d_{1}-\lambda_{1}\right)\left(\bar{\theta}_{2}-\bar{\theta}_{1}\right)}{\left(n_{1}+n_{2}\right)\left(n_{1}\left(d_{2}+\lambda_{2}\right)+n_{2}\left(d_{1}+\lambda_{1}\right)\right)} .
$$

Here our related comments are as follows.

\footnotetext{
${ }^{38}$ According to Cassola et al. (2013) average bid shading almost tripled after the turmoil in August 2007 in the ECB liquidity auctions.
} 
- When $d_{1}+\lambda_{1}=d_{2}+\lambda_{2}=d+\lambda$ (as in the symmetric case), the expected discount is $(d+\lambda) Q /\left(n_{1}+n_{2}\right)$.

- The first term is always positive provided $Q>0$, whereas the second term is positive whenever $\left(d_{2}+\lambda_{2}-d_{1}-\lambda_{1}\right)\left(\bar{\theta}_{2}-\bar{\theta}_{1}\right)>0$. Therefore, the expected discount is lower whenever the group that values the asset more highly $\left(\bar{\theta}_{2}>\bar{\theta}_{1}\right)$ has a lower "total transaction cost" $\left(d_{2}+\lambda_{2}<d_{1}+\lambda_{1}\right)$.

- If group 1 ex ante values the asset more $\left(\bar{\theta}_{1}>\bar{\theta}_{2}\right)$, has higher transaction costs $\left(\lambda_{1}>\lambda_{2}\right)$, is more numerous $\left(n_{1}>n_{2}\right)$, and is less informed $\left(\sigma_{\varepsilon_{1}}^{2}>\sigma_{\varepsilon_{2}}^{2}\right)$, then Corollary 2 shows that $d_{1}+\lambda_{1}>d_{2}+\lambda_{2}$ whenever (a) the differences between groups are due mostly to transaction costs and (b) $\lambda_{1} / \lambda_{2}$ is high enough. In this case, both terms are positive and so the expected discount is positive. Yet, if both groups have similar transactions costs, then the two terms in (56) have opposite signs. In particular, we expect a negative discount when $Q$ is low.

\section{Effects of Integrating Separated Markets}

We compare the expected total surplus of our setup with a framework with two separated markets, the market 1 and the market 2 . In market $i$ all the buyers $\left(n_{i}\right)$ are of type $i$ and the quantity supplied is $n_{i} Q /\left(n_{1}+n_{2}\right)$. In this last framework, given that all the individuals are identical, the market clearing condition implies that the equilibrium quantities in market $i$ are given by $Q /\left(n_{i}+n_{j}\right)$. Hence, the expected total surplus in market $i$, denoted by $\mathbb{E}[T S]_{\text {Market } i}$, satisfies

$$
\mathbb{E}[T S]_{\text {Market } i}=\frac{n_{i} \bar{\theta}_{i}}{n_{1}+n_{2}} Q-\frac{\lambda_{i} n_{i}}{\left(n_{1}+n_{2}\right)^{2}} \frac{Q^{2}}{2}
$$

and, consequently, the sum of expected total surplus in this setting

$$
\mathbb{E}[T S]_{\text {Market } 1}+\mathbb{E}[T S]_{\text {Market } 2}=\frac{n_{1} \bar{\theta}_{1}+n_{2} \bar{\theta}_{2}}{n_{1}+n_{2}} Q-\frac{n_{1} \lambda_{1}+n_{2} \lambda_{2}}{\left(n_{1}+n_{2}\right)^{2}} \frac{Q^{2}}{2} .
$$

On the other hand, in our setup (the integrated market, denoted IM) the expected total surplus is given by

$$
\begin{aligned}
\mathbb{E}[T S]_{\mathrm{IM}}= & \left(\frac{n_{1}\left(d_{2}+\lambda_{2}\right) \bar{\theta}_{1}+n_{2}\left(d_{1}+\lambda_{1}\right) \bar{\theta}_{2}}{u}-\frac{n_{1} n_{2}\left(\lambda_{1} d_{2}-\lambda_{2} d_{1}\right)\left(\bar{\theta}_{1}-\bar{\theta}_{2}\right)}{u^{2}}\right) Q \\
& -\frac{n_{1} \lambda_{1}\left(d_{2}+\lambda_{2}\right)^{2}+n_{2} \lambda_{2}\left(d_{1}+\lambda_{1}\right)^{2}}{2 u^{2}} Q^{2}+\frac{n_{1} n_{2}\left(n_{2}\left(2 d_{1}+\lambda_{1}\right)+n_{1}\left(2 d_{2}+\lambda_{2}\right)\right)}{2 u^{2}} \mathbb{E}\left[\left(t_{1}-t_{2}\right)^{2}\right],
\end{aligned}
$$


where $u$ and $\mathbb{E}\left[\left(t_{1}-t_{2}\right)^{2}\right]$ are given by (5) and (7), respectively.

At first glance, we can observe that when $Q=0$, it follows that

$$
\mathbb{E}[T S]_{\mathrm{IM}}>\mathbb{E}[T S]_{\text {Market 1 }}+\mathbb{E}[T S]_{\text {Market 2 }}
$$

whenever $\bar{\theta}_{1} \neq \bar{\theta}_{2}$, or when there is an informative private signal (i.e., when $\sigma_{\varepsilon_{1}}^{2}=\sigma_{\varepsilon_{2}}^{2}=\infty$ does not hold). Under this parameter configuration, this inequality also holds if the groups are symmetric (i.e., $\lambda_{1}=\lambda_{2}=\lambda, n_{1}=n_{2}=n, \sigma_{\varepsilon_{1}}^{2}=\sigma_{\varepsilon_{2}}^{2}=\sigma_{\varepsilon}^{2}$, and $\bar{\theta}_{1}=\bar{\theta}_{2}=\bar{\theta}$ ). To see this, note that in this case

$$
\begin{gathered}
\mathbb{E}[T S]_{\mathrm{IM}}=\bar{\theta} Q-\frac{\lambda}{4 n} Q^{2}+\frac{n(2 d+\lambda)}{4(d+\lambda)^{2}} \mathbb{E}\left[\left(t_{1}-t_{2}\right)^{2}\right] \text { and } \\
\mathbb{E}[T S]_{\text {Market 1 }}+\mathbb{E}[T S]_{\text {Market 2 }}=\bar{\theta} Q-\frac{\lambda}{4 n} Q^{2} .
\end{gathered}
$$

Therefore, we can conclude that under symmetry, market integration increases the expected total surplus. In what follows we show that market integration is also good in terms of $\mathbb{E}[T S]$ under perfect competition, even though the groups are asymmetric. Notice that under perfect competition $\left(d_{1}=d_{2}=0\right)$, we have that

$$
\mathbb{E}[T S]_{\mathrm{IM}}=\frac{1}{n_{1} \lambda_{2}+n_{2} \lambda_{1}}\left(\left(n_{1} \lambda_{2} \bar{\theta}_{1}+n_{2} \lambda_{1} \bar{\theta}_{2}\right) Q-\frac{\lambda_{1} \lambda_{2}}{2} Q^{2}+\frac{n_{1} n_{2}}{2} \mathbb{E}\left[\left(t_{1}-t_{2}\right)^{2}\right]\right) .
$$

Hence,

$$
\begin{gathered}
\mathbb{E}[T S]_{\mathrm{IM}}-\left(\mathbb{E}[T S]_{\text {Market } 1}+\mathbb{E}[T S]_{\text {Market } 2}\right)= \\
=\frac{n_{1} n_{2}}{n_{1} \lambda_{2}+n_{2} \lambda_{1}}\left(\frac{\left(\bar{\theta}_{1}-\bar{\theta}_{2}\right)\left(\lambda_{2}-\lambda_{1}\right)}{n_{1}+n_{2}} Q+\frac{\left(\lambda_{2}-\lambda_{1}\right)^{2}}{2\left(n_{1}+n_{2}\right)^{2}} Q^{2}+\frac{\mathbb{E}\left[\left(t_{1}-t_{2}\right)^{2}\right]}{2}\right),
\end{gathered}
$$

which is a convex function in $Q$. Notice that, if $\left(\bar{\theta}_{1}-\bar{\theta}_{2}\right)\left(\lambda_{2}-\lambda_{1}\right) \geq 0$, it follows that

$$
\mathbb{E}[T S]_{\mathrm{IM}} \geq \mathbb{E}[T S]_{\text {Market } 1}+\mathbb{E}[T S]_{\text {Market } 2}
$$

with an equality only holds when $\bar{\theta}_{1}=\bar{\theta}_{2}, \lambda_{1}=\lambda_{2}$, and $\sigma_{\varepsilon_{1}}^{2}=\sigma_{\varepsilon_{2}}^{2}=\infty$. Otherwise, if $\left(\bar{\theta}_{1}-\bar{\theta}_{2}\right)\left(\lambda_{2}-\lambda_{1}\right)<0$, the expected total surplus is also larger in the integrated market. In order to show this, we examine the value of $\mathbb{E}[T S]_{\mathrm{IM}}-\left(\mathbb{E}[T S]_{\text {Market } 1}+\mathbb{E}[T S]_{\text {Market } 2}\right)$ at the minimum, which is $Q=\frac{n_{1}+n_{2}}{\lambda_{1}-\lambda_{2}}\left(\bar{\theta}_{1}-\bar{\theta}_{2}\right)$. Direct computation yields that this function at its minimum is strictly positive and, therefore, for all $Q \geq 0$, this difference is also strictly positive. Therefore, we can conclude that, under perfect competition the following inequality holds

$$
\mathbb{E}[T S]_{\mathrm{IM}} \geq \mathbb{E}[T S]_{\text {Market 1 }}+\mathbb{E}[T S]_{\text {Market 2 }},
$$


and the equality is satisfied when $\bar{\theta}_{1}=\bar{\theta}_{2}, \lambda_{1}=\lambda_{2}$, and $\sigma_{\varepsilon_{1}}^{2}=\sigma_{\varepsilon_{2}}^{2}=\infty$.

To find the reverse result, we have to restrict our attention to a setup with strategic behavior. Moreover, we assume that $\bar{\theta}_{1}=\bar{\theta}_{2}=\bar{\theta}$ and $\sigma_{\varepsilon_{1}}^{2}=\sigma_{\varepsilon_{2}}^{2}=\infty$. Then, it follows that

$$
\begin{gathered}
\mathbb{E}[T S]_{\mathrm{IM}}=\bar{\theta} Q-\frac{n_{1} \lambda_{1}\left(d_{2}+\lambda_{2}\right)^{2}+n_{2} \lambda_{2}\left(d_{1}+\lambda_{1}\right)^{2}}{u^{2}} \frac{Q^{2}}{2} \text { and } \\
\mathbb{E}[T S]_{\text {Market 1 }}+\mathbb{E}[T S]_{\text {Market 2 }}=\bar{\theta} Q-\frac{n_{1} \lambda_{1}+n_{2} \lambda_{2}}{\left(n_{1}+n_{2}\right)^{2}} \frac{Q^{2}}{2} .
\end{gathered}
$$

Therefore, in this case

$$
\begin{gathered}
\mathbb{E}[T S]_{\mathrm{IM}}-\left(\mathbb{E}[T S]_{\text {Market } 1}+\mathbb{E}[T S]_{\text {Market 2 }}\right)= \\
n_{1} n_{2}\left(d_{1}+\lambda_{1}-\lambda_{2}-d_{2}\right) \frac{2\left(d_{1}+\lambda_{1}\right)\left(\lambda_{1}-\lambda_{2}\right)\left(n_{1}+n_{2}\right)+\left(n_{1}\left(2 \lambda_{1}-\lambda_{2}\right)+\lambda_{1} n_{2}\right)\left(d_{2}+\lambda_{2}-d_{1}-\lambda_{1}\right)}{\left(n_{1}+n_{2}\right)^{2} u^{2}} \frac{Q^{2}}{2} .
\end{gathered}
$$

In particular, whenever $\lambda_{1}>\lambda_{2}, d_{1}+\lambda_{1}<d_{2}+\lambda_{2}$, and $Q \neq 0$, it follows that

$$
\mathbb{E}[T S]_{\mathrm{IM}}<\mathbb{E}[T S]_{\text {Market } 1}+\mathbb{E}[T S]_{\text {Market } 2}
$$

To intuitively understand this result notice that, when bidders do not have private information and ex-ante identically value the asset $\left(\bar{\theta}_{1}=\bar{\theta}_{2}=\bar{\theta}\right)$, the individual equilibrium quantity for the group $i$ can be written as

$$
\frac{Q}{n_{i}+n_{j}}-\frac{\left(d_{i}+\lambda_{i}-d_{j}-\lambda_{j}\right) Q n_{j}}{\left(n_{i}+n_{j}\right) u}
$$

Then, when $d_{2}+\lambda_{2}>d_{1}+\lambda_{1}$, group 2 which has lower transaction costs $\left(\lambda_{1}>\lambda_{2}\right)$, in equilibrium, obtains a lower quantity in the integrated market with respect to the case with separated markets. This result is due to the fact that in the integrated market the agents of group 2 reduce their demand strategically and, as a result their equilibrium quantities are lower, while the agents with higher transaction costs get a higher quantity. All this leads to a lower expected total surplus in the integrated market.

Finally, we end this analysis with another case (now with incomplete information) in which market integration also reduces the expected total surplus. Suppose that $\bar{\theta}_{1}=\bar{\theta}_{2}=\bar{\theta}, \lambda_{1}=$ $\lambda_{2}=\lambda, n_{1}=n_{2}=n, \rho \neq 0$, and $\widehat{\sigma}_{\varepsilon_{1}}^{2} \neq \widehat{\sigma}_{\varepsilon_{2}}^{2}$. We have that

$$
\mathbb{E}[T S]_{\text {Market } 1}+\mathbb{E}[T S]_{\text {Market } 2}=\bar{\theta} Q-\frac{\lambda}{4 n} Q^{2},
$$

and

$$
\mathbb{E}[T S]_{\mathrm{IM}}=\bar{\theta} Q-\frac{\lambda}{2 n} \frac{\left(d_{2}+\lambda\right)^{2}+\left(d_{1}+\lambda\right)^{2}}{\left(d_{1}+d_{2}+2 \lambda\right)^{2}} Q^{2}+n \frac{d_{1}+d_{2}+\lambda}{\left(d_{1}+d_{2}+2 \lambda\right)^{2}} \mathbb{E}\left[\left(t_{1}-t_{2}\right)^{2}\right]
$$


Suppose that $Q$ is large enough, the comparison of the previous two expressions is simply reduced to the comparison of the coefficients associated with $Q^{2}$ (in absolute values). ${ }^{39}$ Note that

$$
\frac{\lambda}{2 n} \frac{\left(d_{2}+\lambda\right)^{2}+\left(d_{1}+\lambda\right)^{2}}{\left(d_{1}+d_{2}+2 \lambda\right)^{2}}-\frac{\lambda}{4 n}=\frac{\lambda\left(d_{1}-d_{2}\right)^{2}}{4 n\left(d_{1}+d_{2}+2 \lambda\right)^{2}}>0 .
$$

This implies that for high enough values of $Q$,

$$
\mathbb{E}[T S]_{\mathrm{IM}}<\mathbb{E}[T S]_{\text {Market 1 }}+\mathbb{E}[T S]_{\text {Market 2 }} \text {. }
$$

\section{References}

Akgün, U. (2004): "Mergers with supply functions," Journal of Industrial Economics, 52 (4), 535-546.

Anderson, E. and X. Hu (2008): "Finding supply function equilibria with asymmetric firms," Operations Research, 56 (3), 697-711.

Armantier, O., and E. Sbaï (2006): "Estimation and comparison of treasury auction formats when bidders are asymmetric," Journal of Applied Econometrics, 21, 745-779.

Arnone, M., and G. Iden (2003): "Primary dealers in government securities: policy issues and selected countries' experience," IMF Working Paper 03/45.

Athey, S. (2001): "Single crossing properties and the existence of pure strategy equilibria in games of incomplete information," Econometrica, 69 (4), 861-890.

Athey, S., D. Coey and J. Levin (2013): "Set-asides and subsidies in auctions," American Economic Journal: Microeconomics, 5 (1), 1-27.

Ausubel, L. M., P. Cramton, M. Pycia, M. Rostek, and M. Weretka (2014): "Demand reduction and inefficiency in multi-unit auctions," The Review of Economic Studies, 81 (4), 1366-1400.

Babus, A. and P. Kondor (2017):"Trading and Information Diffusion in Over-the-Counter Markets", unpublished working paper. London School of Economics, London, UK.

Back, K., and J.F. Zender (1993): "Auctions of divisible goods: on the rationale for the Treasury experiment," The Review of Financial Studies, 6, 733-764.

Bajari, P. (1998): "A structural econometric model of the first price sealed bid auction with asymmetric bidders," Manuscript, Stanford University.

Bergemann, D., T. Heumann, and S. Morris (2015): "Information and market power," Cowles Foundation working paper no. 2017.

\footnotetext{
${ }^{39}$ Assume that $\bar{\theta}$ is high enough so that expected total surplus is positive.
} 
Bindseil, U., K. Nyborg, and I. Strebulaev (2009): "Bidding and performance in repo auctions: Evidence from ECB open market operations," Journal of Money, Credit and Banking, 41 (7), 1391-1421.

Bjonnes, G.H. (2001): "Winner's curse in discriminatory price auctions: Evidence from the Norwegian Treasury bill auctions," SIFR Research Report Series 3, Institute for Financial Research.

Borenstein, S., J. Bushnell, and F. Wolak (2002): "Measuring market inefficiencies in California's restructured wholesale electricity market," American Economic Review, 92 (5), 1376-1405.

Boyarchenko, N., D.O. Lucca, and L. Veldkamp (2015): "Intermediaries as information aggregators: an application to US Treasury Auctions," Federal Reserve Bank of New York Staff Report no. 726.

Brandts, J., Reynolds, S. and A. Schram (2014): "Pivotal suppliers and market power in experimental supply function competition," Economic Journal, 124 (579), 887-916.

Bustos-Salvagno, J. (2015): "Bidding behavior in the Chilean electricity market," Energy Economics, 51, 288-299.

Cantillon, E. (2008): "The effect of bidders' asymmetries on expected revenue in auctions," Games and Economic Behavior, 62 (1), 1-25.

Cao, M., and D. Lu (2004): "Information and winning: evidence from the 3-month Canadian treasury auctions," Working paper.

Cassola, N., A. Hortaçsu, and J. Kastl (2013): "The 2007 subprime market crisis through the lens of European Central Bank auctions for short-term funds," Econometrica, 81 (4), 1309-1345.

Ciarreta, A., and M.P. Espinosa (2010): "Supply function competition in the Spanish wholesale electricity market," Energy Journal, 31 (4), 137-157.

Cramton, P., and S. Stoft (2006): "Uniform price auctions in electricity markets," U. Maryland Working paper.

Cramton, P., and S. Stoft (2007): "Why we need to stick with uniform price auctions in electricity markets," Electricity Journal, 20 (1), 26-37.

Crawford, G., J. Crespo, and H. Tauchen (2007): "Bidding asymmetries in multi-unit auctions: Implications of bid function equilibria in the British spot market for electricity," International Journal of Industrial Organization, 25 (6), 1233-1268.

Du, S. and H. Zhu (2015): "Welfare and optimal trading frequency in dynamic double auctions," Working paper.

Du, S. and H. Zhu (2016): "Bilateral trading in divisible double auctions," Working paper. 
Engelbrecht-Wiggans, R., J.A. List, and D. Lucking-Reiley (2006): "Demand reduction in multi-unit auctions with varying numbers of bidders: Theory and field experiments," International Economic Review, 47, 203-231.

European Commission (2007): "DG Competition report on energy sector inquiry," 10 January 2007, SEC (2006) 1724, Brussels, Commission of the European Communities.

Ewerhart, C., N. Cassola, and N. Valla (2010): "Declining valuations and equilibrium bidding in central bank refinancing operations," International Journal of Industrial Organization, 28, $30-43$.

Goldstein, I. and L. Yang (2015): "Information diversity and complementarities in trading and information acquisition," The Journal of Finance 70 (4), 1723-1765.

Green, R., and D. Newbery (1992): "Competition in the British electricity spot market," Journal of Political Economy, 100, 929-953.

Grossman, S., and J. Stiglitz (1980): "On the impossibility of informationally efficient markets," American Economic Review, 70, 393-408.

Hafalir, I., and V. Krishna (2008): "Asymmetric auctions with resale," American Economic Review, 98, 87-112.

Hamao, M., and N. Jegadeesh (1998): "An analysis of bidding in the Japanese government bond auctions," The Journal of Finance 53 (2), 755-772.

Holmberg, P., D. Newbery, and D. Ralph (2013): "Supply function equilibria: Step functions and continuous representations," Journal of Economic Theory, 148 (4), 1509-1551.

Holmberg, P., and F. Wolak (2015): "Electricity markets: Designing auctions where suppliers have uncertain costs," IFN working paper no. 1099.

Hortaçsu, A., and J. Kastl (2012): "Valuing dealers' informational advantage: a study of Canadian Treasury auctions," Econometrica, 80 (6), 2511-2542.

Hortaçsu, A., J. Kastl, and A. Zhang (2016): "Bid shading and bidder surplus in the US Treasury auction system," Working Paper.

Hortaçsu, A., and D. McAdams (2010): "Mechanism choice and strategic bidding in divisible good auctions: An empirical analysis of the Turkish Treasury auction market," Journal of Political Economy, 118 (5), 833-865.

Hortaçsu, A., and S. Puller (2008): "Understanding strategic bidding in multi-unit auctions: A case study of the Texas electricity spot market," RAND Journal of Economics, 39 (1), 86-114.

Hubbard, T., and R. Kirkegaard (2015): "Asymmetric Auctions with More Than Two Bidders," Working Papers 1502, University of Guelph, Department of Economics and Finance. 
Joskow, P., and E. Kahn (2002): "A quantitative analysis of pricing behavior in California's wholesale electricity market during summer 2000," The Energy Journal, 23 (4), 1-35.

Kagel, J.H., and D. Levin (2001): "Behavior in multi-unit demand auctions: Experiments with uniform price and dynamic Vickrey auctions," Econometrica, 69, 413-454.

Kastl, J. (2011): "Discrete bids and empirical inference in divisible good auctions," The Review of Economic Studies, 78 (3), 974-1014.

Klemperer, P., and M. Meyer (1989): "Supply function equilibria in oligopoly under uncertainty," Econometrica, 57, 1243-1277.

Kyle, A. (1985): "Continuous auctions and insider trading," Econometrica, 53, 1315-1335.

Kyle, A. (1989): "Informed speculation with imperfect competition," The Review of Economic Studies, 56, 317-356.

Lambert, N., M. Ostrovsky, and M. Pavnov (2016): "Strategic trading in informationally complex environments," Working paper.

Lebrun, B. (1996): "Existence of an equilibrium in first price auctions," Economic Theory, $7(3), 421-443$.

Lebrun, B. (1999): "First price auctions in the asymmetric $\mathrm{N}$ bidder case," International Economic Review, 40 (1), 125-142.

Loertscher, S. and L. Marx (2016): "Auctions with bid credits and resale", Working Paper.

Lopomo, G., L. Marx, D. McAdams, and B. Murray (2011): "Carbon allowance auction design: an assessment of options for the United States," Review of Environmental Economics and Policy, 5 (1) 25-43.

Malvey, P.F., and C.F. Archibald (1998): "Uniform-price auctions: Update of the Treasury experience," US Treasury.

Maskin, E., and J.G. Riley (2000a): "Asymmetric auctions," The Review of Economic Studies, 67 (3), 413-438.

Maskin, E., and J.G. Riley (2000b): "Equilibrium in sealed high bid auctions," The Review of Economic Studies, 67 (3), 439-454.

Maskin, E., and J.G. Riley (2003): "Uniqueness in sealed high bid auctions," Games and Economic Behavior 45 (2), 395-409.

McAdams, D. (2003): "Isotone equilibrium in games of incomplete information," Econometrica 71 (4), 1191-1214.

McAdams, D. (2006): "Monotone equilibrium in multi-unit auctions," The Review of Economic Studies 73, 1039-1056. 
Pai, M. and R. Vohra (2012): "Auction design with fairness concerns: subsidies vs. setasides," Discussion Paper, Center for Mathematical Studies in Economics and Management Science, Northwestern University.

Pesendorfer, M. (2000): "A study of collusion in first-price auctions," The Review of Economic Studies, 67 (3), 381-411.

Porter, R.H., and J.D. Zona (1999): "Ohio school milk markets: An analysis of bidding," RAND Journal of Economics, 30 (2), 263-288.

Pycia, M., and K. Woodward (2016): "Pay-as-bid. Selling divisible goods," Working paper.

Reny, P. (2011): "On the existence of monotone pure-strategy equilibria in Bayesian games," Econometrica 79 (2), 499-553.

Reny, P., and S. Zamir (2004): "On the existence of pure strategy monotone equilibria in asymmetric first-price auctions," Econometrica, 72 (4), 1105-1125.

Rostek, M., and M. Weretka (2012): "Price inference in small markets," Econometrica 80 (2), 687-711.

Sadzik, T., and P. Andreyanov (2016): "Robust mechanism design of exchange," Working paper.

Umlauf, S.R. (1993): "An empirical study of the Mexican Treasury bill auction," Journal of Financial Economics, 33, 313-340.

Vives, X. (2010): "Asset auctions, information, and liquidity," Journal of the European Economic Association, 8 (2-3), 467-477.

Vives, X. (2011): "Strategic supply function competition with private information," Econometrica, 79 (6), 1919-1966.

Vives, X. (2014): "On the possibility of informationally efficient markets," Journal of the European Economic Association, 12 (5), 1200-1239.

Vives, X. (2017): "Endogenous public information and welfare in market games," Review of Economic Studies 84 (2), 935-963.

Wang, J.J.D., and J.F. Zender (2002): "Auctioning divisible goods," Economic Theory, 19 (4), 673-705.

Wilson, R. (1979): "Auctions of shares," Quarterly Journal of Economics, 93, 675-698.

Wolfram, C. (1998): "Strategic bidding in a multiunit auction: An empirical analysis of bids to supply electricity in England and Wales," RAND Journal of Economics, 29, 703-725. 


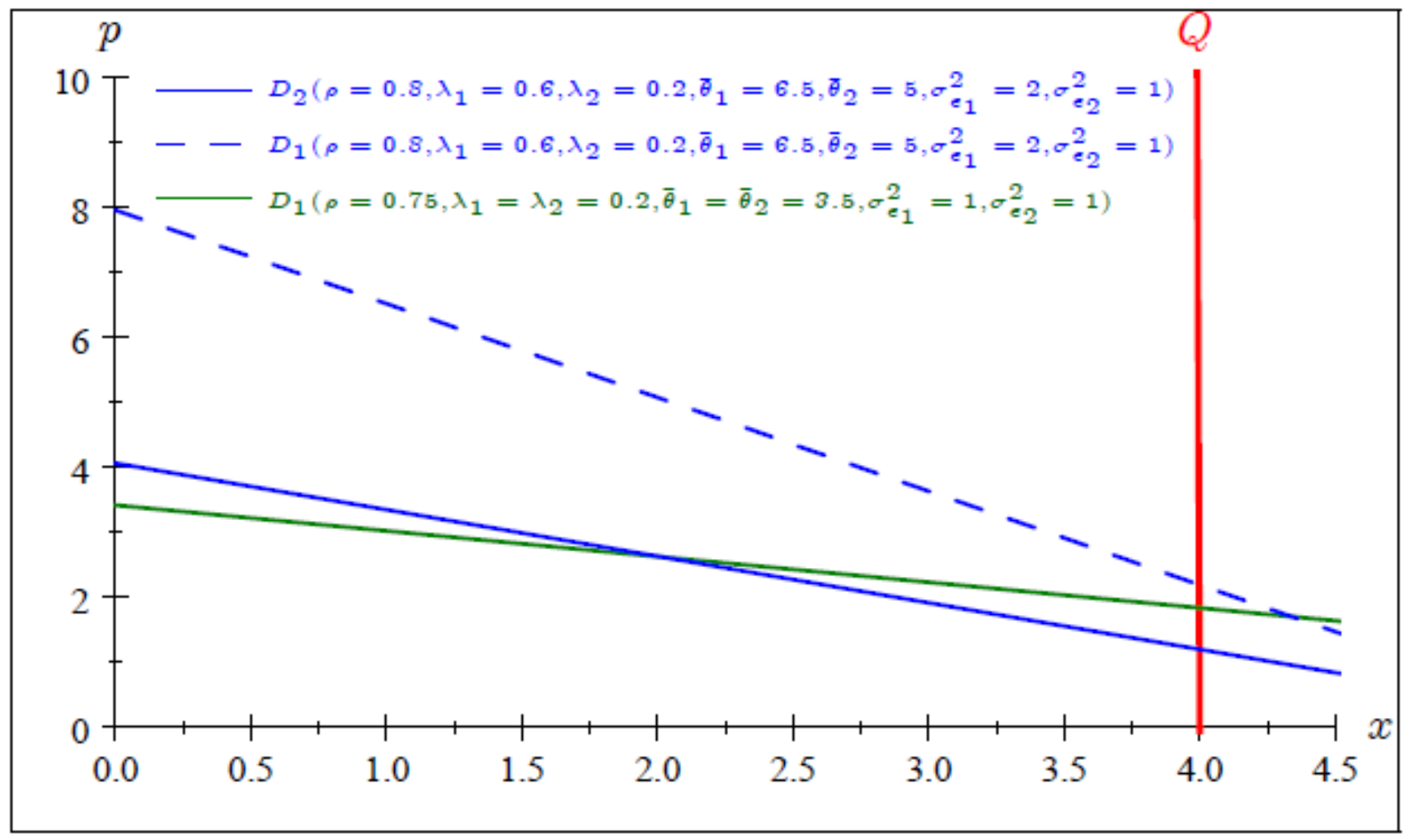

Figure 1: Comparative statics on demand functions $n_{i}=5 ; s_{i}=\bar{\theta}_{i} ; \sigma_{\theta}^{2}=5 ; Q=4$. The green line corresponds to the optimal demand curve for a bidder in the symmetric case, while the blue lines represent the optimal demand curves for each group when the correlation coefficient among valuations, and the transactions cost and the noise in the signal for group 1 have increased. 


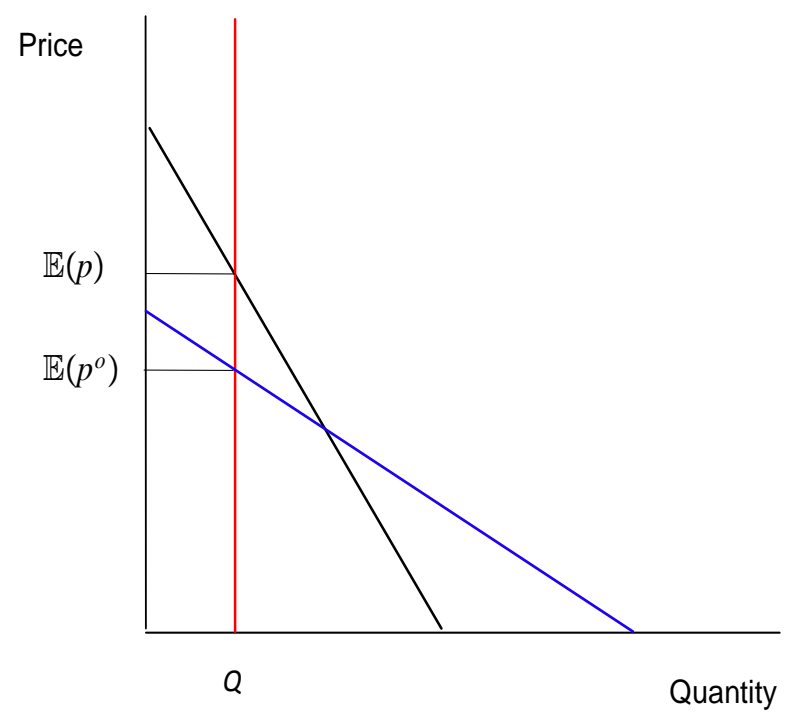

Figure 2: Comparison of expected prices under imperfect and perfect competition. The blue (black) line represents the expected demand curve under perfect (imperfect competition) competition, while the red one represents the supply curve. 